Florida International University FIU Digital Commons

\title{
A method for enhancing digital information displayed to computer users with visual refractive errors via spatial and spectral processing
}

Miguel Alonso Jr.

Florida International University

DOI: $10.25148 /$ etd.FI13101606

Follow this and additional works at: https://digitalcommons.fiu.edu/etd

Part of the Electrical and Computer Engineering Commons

\section{Recommended Citation}

Alonso, Miguel Jr., "A method for enhancing digital information displayed to computer users with visual refractive errors via spatial and spectral processing" (2007). FIU Electronic Theses and Dissertations. 1112.

https://digitalcommons.fiu.edu/etd/1112 
Miami, Florida

\section{A METHOD FOR ENHANCING DIGITAL INFORMATION DISPLAYED TO COMPUTER USERS WITH VISUAL REFRACTIVE ERRORS VIA SPATIAL AND SPECTRAL PROCESSING}

A dissertation submitted in partial fulfillment of the requirements for the degree of DOCTOR OF PHILOSOPHY in ELECTRICAL ENGINEERING

by

Miguel Alonso Jr 
To: Dean Vish Prasad

College of Engineering and Computing

This dissertation, written by Miguel Alonso Jr, and entitled A Method for Enhancing Digital Information Displayed to Computer Users with Visual Refractive Errors via Spatial and Spectral Processing, having been approved in respect to style and intellectual content, is referred to you for judgment.

We have read this dissertation and recommend that it be approved.

Malek Adjouadi

Roberto Panepucci

Shu-Ching Chen

Julie A. Jacko

Armando Barreto, Major Professor

Date of Defense: May 25, 2007

The dissertation of Miguel Alonso Jr is approved.

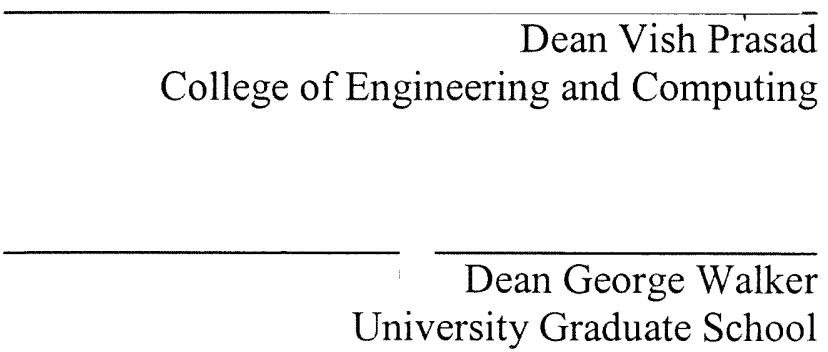

Florida International University, 2007 
(C) Copyright 2007 by Miguel Alonso Jr

All rights reserved. 


\section{DEDICATION}

I dedicate this $\mathrm{Ph}$. $\mathrm{D}$. dissertation to my loving girlfriend and family, for without their enduring support, I could not have completed this endeavor. 


\section{ACKNOWLEDGMENTS}

I would like to thank my major Professor, Dr. Armando Barreto, for his excellent guidance and friendship. I would also like to acknowledge the National Science Foundation grants IIS-0308155, CNS-0520811, HRD-0317692 and CNS-0426125. During the majority of the development of my research I was supported by an NSF Graduate Research Fellowship. I completed the final year of my dissertation thanks to the FIU Dissertation Year Fellowship. Finally, I would like to thank my committee members Dr. Malek Adjouadi, Dr. Roberto Panepucci, Dr. Julie Jacko, and Dr. Shu-Ching Chen, as well as Dr. Gualberto Cremades for their expertise and advice. 


\section{ABSTRACT OF THE DISSERTATION \\ A METHOD FOR ENHANCING DIGITAL INFORMATION DISPLAYED TO \\ COMPUTER USERS WITH VISUAL REFRACTIVE ERRORS VIA SPATIAL AND \\ SPECTRAL PROCESSING}

by

Miguel Alonso Jr

Florida International University, 2007

Miami, Florida

Professor Armando Barreto, Major Professor

This research pursued the conceptualization, implementation, and verification of a system that enhances digital information displayed on an LCD panel to users with visual refractive errors. The target user groups for this system are individuals who have moderate to severe visual aberrations for which conventional means of compensation, such as glasses or contact lenses, does not improve their vision.

This research is based on a priori knowledge of the user's visual aberration, as measured by a wavefront analyzer. With this information it is possible to generate images that, when displayed to this user, will counteract his/her visual aberration. The method described in this dissertation advances the development of techniques for providing such compensation by integrating spatial information in the image as a means to eliminate some of the shortcomings inherent in using display devices such as monitors or LCD panels. Additionally, physiological considerations are discussed and integrated into the method for providing said compensation. 
In order to provide a realistic sense of the performance of the methods described, they were tested by mathematical simulation in software, as well as by using a single-lens high resolution $\mathrm{CCD}$ camera that models an aberrated eye, and finally with human subjects having various forms of visual aberrations. Experiments were conducted on these systems and the data collected from these experiments was evaluated using statistical analysis.

The experimental results revealed that the pre-compensation method resulted in a statistically significant improvement in vision for all of the systems. Although significant, the improvement was not as large as expected for the human subject tests. Further analysis suggest that even under the controlled conditions employed for testing with human subjects, the characterization of the eye may be changing. This would require real-time monitoring of relevant variables (e.g. pupil diameter) and continuous adjustment in the pre-compensation process to yield maximum viewing enhancement. 


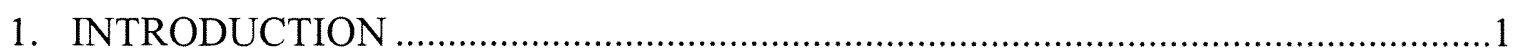

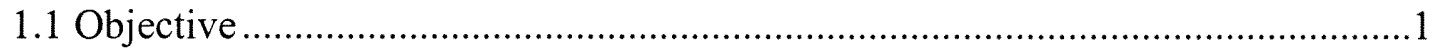

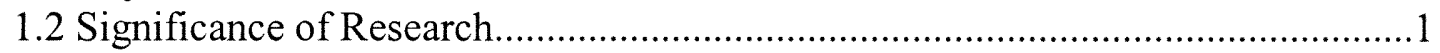

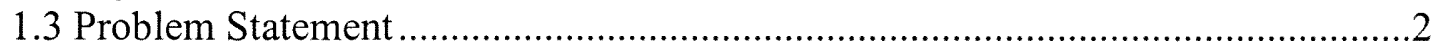

1.4 Introduction to the Point Spread Function of the Human Eye................................ 3

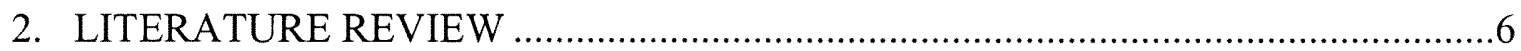

2.1 Prior research in image enhancement for low vision..........................................6

2.2 Using Wavefront Analysis for Compensation of Visual Aberrations ....................9

2.3 Current methods of Compensating for the PSF ..................................................

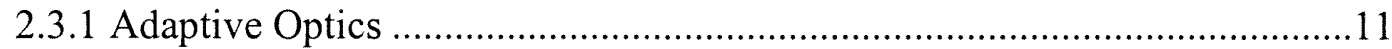

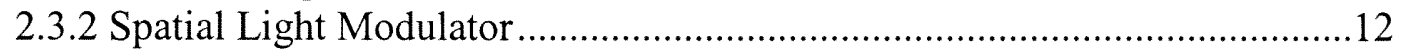

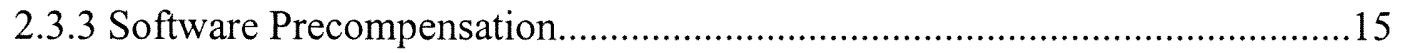

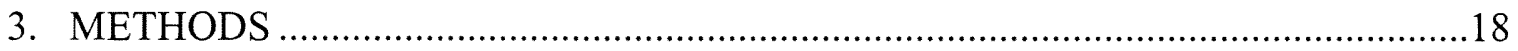

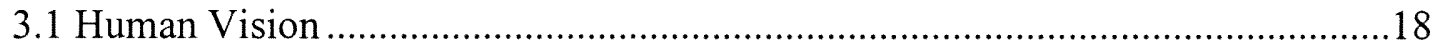

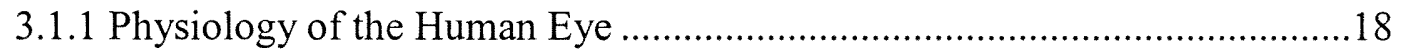

3.1.2 The Eye as a Dynamic Optical System .....................................................19

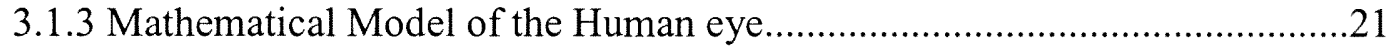

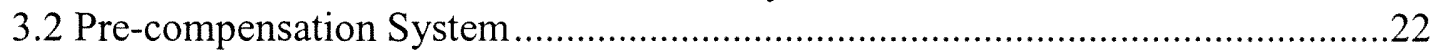

3.2.1 Spectral Processing for Inverse Solution .................................................22

3.2.2 Spatial Processing for Feature Extraction...................................................27

3.2.3 Integration of Spatial and Spectral Modalities.............................................40

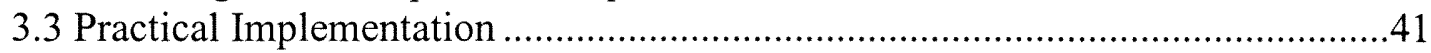

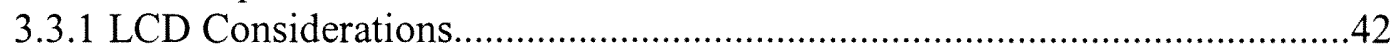

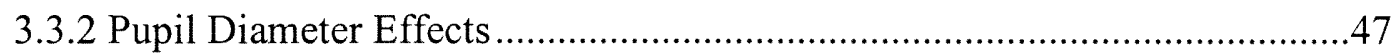

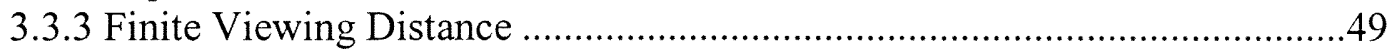

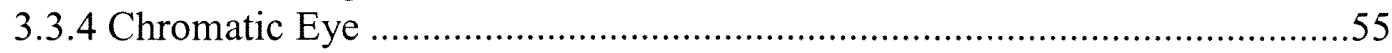

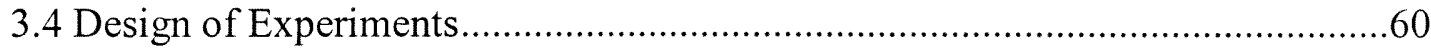

3.4.1 Software Simulation Experimental Design...................................................60

3.4.2 Artificial Eye Experimental Design...........................................................63

3.4.3 Human Subject Experimental Design .........................................................65

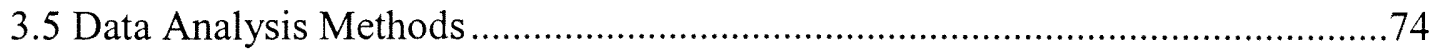

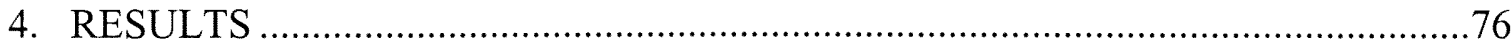

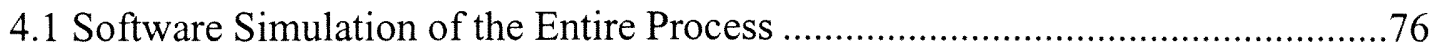

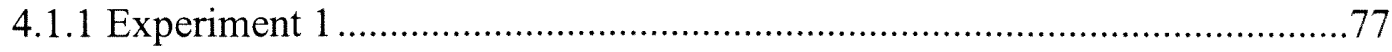

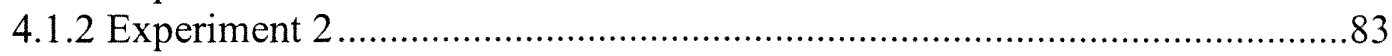

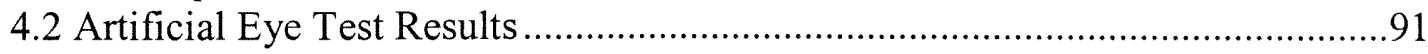

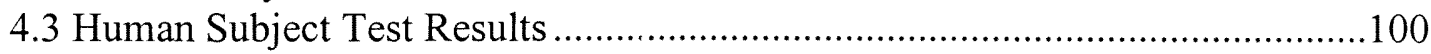

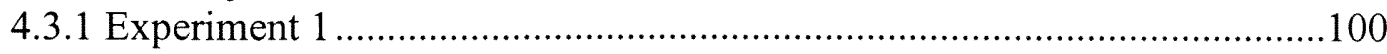




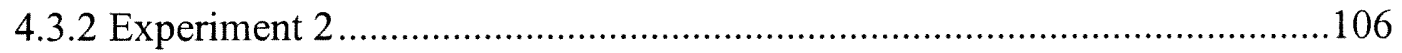

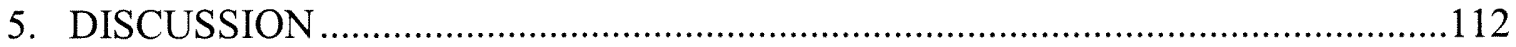

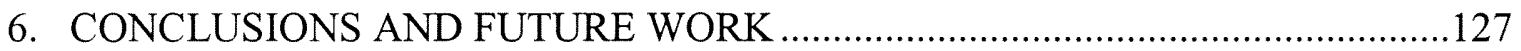

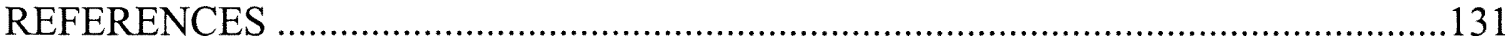

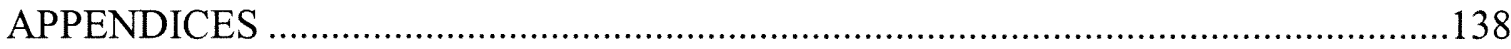

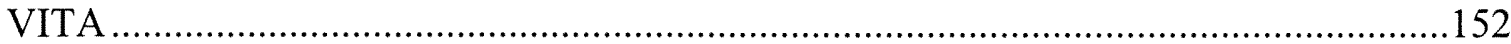




\section{LIST OF TABLES}

TABLE

PAGE

Table 3-1 LCD wavelengths and their corresponding correction factors 57

Table 3-2 Factors for software experiment 1 61

Table 3-3 Factors for software experiment 2 63

Table 3-4 Factors for artificial eye experiment. 65

Table 3-5 Subject number, group, and prescription. 66

Table 3-6 The log of the Minimum Angle Resolved (logMAR) scale, minimum angles of resolution, and equivalent decimal $\mathrm{V}$ and Snellen distance acuities 68

Table 3-7 Factors for human subjects experiment 1 71

Table 3-8 Factors for human subject experiment 2 74

Table 4-1 Software Test: Experiment 1, Outliers and Replacements 78

Table 4-2 Software Test: Experiment 1, Normality Test on Studentized Residuals

Table 4-3 Software Test: Experiment 1, Levene's Test for homogeneity of cell variances. 80

Table 4-4 ANOVA Table for Software Test: Experiment 1 81

Table 4-5 Software Test: Experiment 1 Contrast on Size 81

Table 4-6 Software Test: Experiment 1 Contrast on Method 82

Table 4-7 Software Test: Experiment 1, Estimated Marginal Mean for size 82

Table 4-8 Software Test: Experiment 1, Estimated Marginal Mean for method. 82

Table 4-9 Software Test: Experiment 1, S-N-K for size 82

Table 4-10 Software Test: Experiment 2, Outliers 84

Table 4-11 Software Test: Experiment 2, Skewness and Kurtosis of residuals 86 
Table 4-12 Software Test: Experiment 2, Levene's test of homogeneity of cell variances

Table 4-13 Software Test: Experiment 2, ANOVA Table 87

Table 4-14 Software Test: Experiment 2, Contrast table for icon factor 88

Table 4-15 Software Test: Experiment 2, Contrast table for size factor 89

Table 4-16 Software Test: Experiment 2, Contrast table for method factor. 89

Table 4-17 Software Test: Experiment 2, Estimated marginal means for method factor

Table 4-18 Software Test: Experiment 2, S-N-K table for icon factor. 90

Table 4-19 Software Test: Experiment 2, S-N-K for size factor 90

Table 4-20 Software Test: Experiment 2, Estimated marginal means for icon factor. 90

Table 4-21 Software Test: Experiment 2, Estimated marginal means for size factor 90

Table 4-22 Artificial Eye Test: Outliers 93

Table 4-23 Artificial Eye Test: Skewness and Kurtosis for the

Studentized residuals 95

Table 4-24 Artificial Eye Test: Levene's test for homogeneity of variance. 96

Table 4-25 Artificial Eye Test: ANOVA table 96

Table 4-26 Artificial Eye Test: Contrast table for icon factor 97

Table 4-27 Artificial Eye Test: Contrast table for size factor 98

Table 4-28 Artificial Eye Test: Contrast table for method factor 98

Table 4-29 Artificial Eye Test: S-N-K for icon factor...... 99

Table 4-30 Artificial Eye Test: S-N-K for size factor 99

Table 4-31 Artificial Eye Test: Estimated marginal means for icon factor 99

Table 4-32 Artificial Eye Test: Estimated marginal means for size factor. 99

Table 4-33 Artificial Eye Test: Estimated marginal means for method factor 100 
Table 4-34 Human Subject Test: Experiment 1, Outliers

Table 4-35 Human Subject Test: Experiment 1, Skewness and

Kurtosis values for residuals

Table 4-36 Human Subject Test: Experiment 1, Levene Test for

Homogeneity of Variance.

Table 4-37 Human Subject Tests: Experiment 1, ANOVA of Fixed Effects................. 104

Table 4-38 Human Subject Tests: Experiment 1, Covariates ........................................ 104

Table 4-39 Human Subject Tests: Experiment 1, Estimated Means for Groups ............. 104

Table 4-40 Human Subject Test: Experiment 1, E*M Interaction Means...................... 104

Table 4-41 Human Subject Tests: Experiment 1, Pair-wise Comparison of Groups .... 105

Table 4-42 Human Subject Test: Experiment 1, E*G Interaction Means ...................... 105

Table 4-43 Human Subject Test: Experiment 2, Outliers............................................. 106

Table 4-44 Human Subject Test: Experiment 2, Skewness and Kurtosis for the Studentized Residuals 108

Table 4-45 Human Subject Test: Experiment 2, Levene's Test for Homogeneity of Variance

Table 4-46 Human Subject Test: Experiment 2, ANOVA Table 109

Table 4-47 Human Subject Test: Experiment 2, Covariates....................................... 110

Table 4-48 Human Subject Test: Experiment 2, Estimated Means for Group ............... 110

Table 4-49 Human Subject Test: Experiment 2, Estimated Means for Size ................. 110

Table 4-50 Human Subject Test: Experiment 2, Estimated Means for Eye ................... 111

Table 4-51 Human Subject Test: Experiment 2, Estimated Means for Method............. 111 


\section{LIST OF FIGURES}

FIGURE

Figure 1-1 Simplified Human Visual Model ............................................................. 4

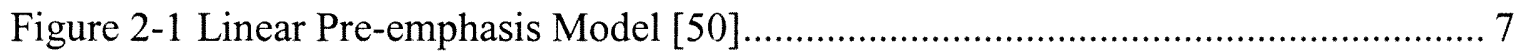

Figure 2-2 Extending the dynamic range in adaptive image enhancement [50]............... 9

Figure 2-3 Adaptive optics wavefront compensation setup [6] .................................. 12

Figure 2-4 Principle of refraction by a spatial light modulator (SLM) [41] .................... 14

Figure 2-5 SLM Compensation Apparatus Setup [36] …......................................... 15

Figure 2-6 Software Pre-compensation process ......................................................... 16

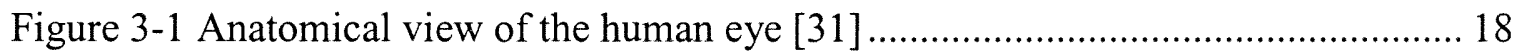

Figure 3-2 Light spread on the retina by (a) an ideal eye and (b) an aberrated eye [31]

Figure 3-3 Example of ideal inverse filter: PSF (top left) Object (top right)

Precompensated Object (Scaled for Display, bottom) …………................................... 24

Figure 3-4 Example of Pre-compensated Folder Icon .................................................. 25

Figure 3-5 Bipolar result of the pre-compensation process (1D slice) …........................ 26

Figure 3-6 Simulation of ideal precompensation process: Icon with blur simulated (bottom left) Precompensated Icon with blur simulated (bottom right)........... 26

Figure 3-7 (a) Shifted and Scaled Precompensated object slice (b)

Retinal Simulation with Object superimposed ……................................................... 28

Figure 3-8 (a) The shifted and scaled precompensated object (b) histogram

equalization of (a) (c) comparison of retinal simulation of (a) and (b) ........................... 29

Figure 3-9 Not all of the information is necessary for reconstruction ............................. 30

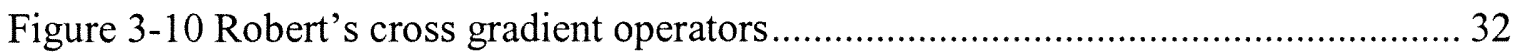

Figure 3-11 Edge detected icon shown in Figure 3-3 ..................................................... 34 
Figure 3-12 Horizontal (left) and Vertical (right) structuring elements

Figure 3-13 Filled in edge detected icon shown in Figure 3-11

Figure 3-14 (a) Original Image (b) Edge-detected (c) Dilated

(d) Filled-in (e) Eroded 38

Figure 3-15 Outline superimposed on original (left) and blurred (right) icon.................. 38

Figure 3-16 (a) Image Mask (b) Mean Mask ……........................................................ 39

Figure 3-17 Black (a) and white (b) artificial backgrounds.......................................... 40

Figure 3-18 Precompensated image (a) and resulting retinal projection (b) ................... 41

Figure 3-19 From the left, Red PSF, Green PSF, Blue PSF of a user with mild myopia

Figure 3-20 Spectral Profile of the Red pixels (top left), Green pixels (top right), Blue pixels (bottom left), Composite (bottom right).

Figure 3-21Red (left), Green (middle), Blue (right) Channels of the

LCD panel captured through a high resolution, machine vision camera 46

Figure 3-22 RGB (i.e. White Background) (left) v.s. RG (i.e. Yellow Background) (right) 46

Figure 3-23 Illustration of how plane waves (infinite distance point source)

(a) and spherical waves (finite distance point source) are focused on the retina 50

Figure 3-24 Geometry used to find wavefront error $(Z)$ due to viewing a point at a distance $(\mathrm{R})$ away from the eye. 51

Figure 3-25 2-D wavefront aberration function for $Z_{\mathrm{def}}=-2 \times 10^{-3} \mathrm{~m}$ with a semi-transparent zero plane 53

Figure 3-26 2-D wavefront aberration function due to viewing an object at $0.5 \mathrm{~m}$ with a semi-transparent plane zero plane 54

Figure 3-27 Chromatic aberrations cause difference of focus (a), magnification (b), and position (c) [31]. 56

Figure 3-28 Wavelength Adjustment Curve. 58

Figure 3-29 Example GUI interface for Human subject VA test 70 
Figure 3-30 Stimulus Screens for the Icon Test. 72

Figure 3-31 Target Screens for the Icon Test 73

Figure 4-1 Three icons with retinal image simulation of normal and precompensated versions 77

Figure 4-2 Software Test: Experiment 1, T Quantile Plot of

Studentized Residuals 78

Figure 4-3 Software Test: Experiment 1, Histogram of Studentized

Residuals with Normal Curve 79

Figure 4-4 Software Test: Experiment 2, Histogram of residuals ................................ 85

Figure 4-5 Software Test: Experiment 2, T-quantile plot of residuals .......................... 86

Figure 4-6 Folder icon with retinal image capture of normal and precompensated versions for each artificial eye lens

Figure 4-7 Artificial Eye Test: Histogram of the residuals............................................. 94

Figure 4-8 Artificial Eye Test: T-quantile plot of the Studentized residuals................... 95

Figure 4-9 Human Subject Test: Experiment 1, Histogram of residuals ....................... 101

Figure 4-10 Human Subject Test: Experiment 1, T-quantile plot of residuals ............... 102

Figure 4-11 Human Subject Test: Experiment 2, Histogram of

Studentized Residuals.

Figure 4-12 Human Subject Test: Experiment 2, T-quantile plot of Residuals 108

Figure 5-1 Software Test: Experiment 2, Interaction plot matrix (I-icon, S-size, M-method). 114

Figure 5-2 Icons used in testing - from left to right, icons one through six 114

Figure 5-3 Software Test: Experiment 2 - Main effects plots (I-icon, S-size, M-method)... 115

Figure 5-4 Artificial Eye Test: Interaction plot matrix 116

Figure 5-5 Artificial Eye Test - Main effects plots 117

Figure 5-6 Human subject tests: Experiment 1 - Interaction plot matrix 118 
Figure 5-7 Human subject tests: Experiment 1 - Histogram of acuity responses

Figure 5-8 Human subject tests: Experiment 2 - Main effects plots

(S-size, M-method)

Figure 5-9 Human subject tests: Experiment 2 - Group*Size, Group*Method,

Size*Method interaction plot matrix

Figure 5-10 Relationship between Defocus, Phi, and Contrast

Figure 5-11 Simulated Retinal Projection of Precompensated image

$(\varphi=0.005$, sphere $=-7$ diopters $)$

Figure 5-12 Simulated Retinal Projection of Precompensated image

$(\varphi=0.005$, sphere $=-3$ diopters $)$ 


\section{CHAPTER 1}

\section{INTRODUCTION}

\subsection{Objective}

The objective of this dissertation is to propose image processing algorithms that will enhance digital information in the form of text displayed on a liquid crystal display panel (LCD panel) to users with visual refractive errors to overcome their loss of visual acuity on a custom basis. The algorithms operate in two modalities, that of the frequency or spectral domain of images, and that of the spatial domain of images. This research also seeks to integrate those two modalities to form what is termed a precompensated object. Additionally, this research ultimately seeks to help the user to perceive the information better, thus a secondary goal is to design experiments that assesses the effectiveness of the methods using first a software model, second a physical model of the eye (i.e. an "artificial eye"), and finally testing the methods with human subjects.

\subsection{Significance of Research}

According to the 1999 Census Bureau's Survey of Income and Program Participation (SIPP), there is an estimated 1.5 million visually impaired computer users. The number of people ages 15 and older with any "limitation in seeing", who report they have access to the Internet, is just over 1.5 million $(1,549,000)$ [22]. 53\% of individuals with general acuity loss report having access to the Internet, compared to only $28 \%$ of individuals with visual impairment extending beyond just general acuity loss [14]. Additionally, an estimated 7 million people in the United States alone have some type of high-order refractive aberration in their eye(s) [18]. In order to remain an active and 
functioning part of society, these individuals need to be able to interact in an efficient manner with graphical user interfaces (GUIs) [14].

Since the proposed method of pre-compensation is entirely digital, i.e., the method is implemented completely in software, any Personal Computer (PC) capable of running a 32-bit operating system with an SVGA graphics card could theoretically be used to deliver the pre-compensation. This research shows promise in benefiting those people who suffer from high-order aberrations, allowing them to potentially interact with any type of digital display more effectively.

\subsection{Problem Statement}

The Research Problem has two main facets:

1. To characterize a fixed and known optical system and provide the compensation for that optical system in order to verify the conceptual integrity of the methods developed before the added complexity of testing with human subject is implemented.

2. In order to test the significance of the compensation, characterize several human eyes and provide a custom compensation for each eye, based on its point spread function (PSF).

According to these two main research facets, the following research questions emerge:

1. Will the proposed method for pre-compensation provide adequate precompensation for an artificial eye if its wavefront aberration function is known a priori? 
2. Will the proposed method for pre-compensation provide adequate precompensation and improve computer interaction for human subjects if their customized wavefront aberration function is known a priori?

\subsection{Introduction to the Point Spread Function of the Human Eye}

The goal of this research is to develop an innovative way of providing enhancement of graphic displays to PC users that have high-order visual aberrations. A number of visual impairments, such as Keratoconus [19], involve high-order visual aberrations that may not be corrected by using glasses or contact lenses. Therefore, the alternative approach proposed here might be particularly valuable for those individuals.

The algorithms developed in this dissertation rely on the linear systems approach to modeling the human visual system, known as Fourier Optics $[44,73]$. The human visual system can be thought of as a linear system having an impulse response $H$. In a linear system, the output of the system is the convolution of the input with the impulse response of the system. The impulse response of an ideal optical system, including the human eye, is a delta function. Thus, if the user is free from any visual aberrations, the impulse response of his/her eye, from here on termed Point Spread Function (PSF), will be a delta, allowing the user to interact more efficiently with the personal computer (PC) via the graphical display. This will result in a clear, undistorted projection of the object onto the retina. If however, the user has a visual aberration, the PSF will not be a delta, and thus the retinal project of the object will be distorted.

Figure 1-1 shows the Linear Shift Invariant (LSI) model used to describe the optical process. The image perceived by the user results from the convolution of an 
object, in this case an image on a graphical display, with the PSF of the user. Under ideal conditions, the perceived image on the retina will be a magnified, but undistorted version of the object. If the user has any type of visual aberration, the resulting image that falls on the retina will be a distorted version of the object.

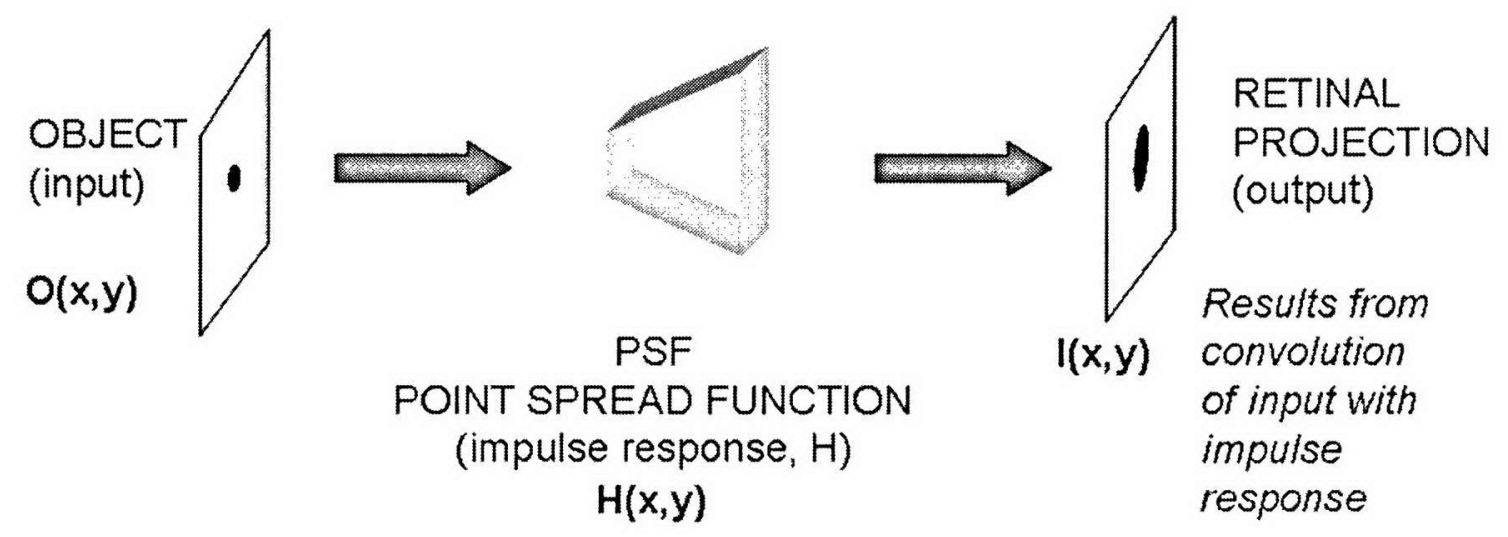

Figure 1-1 Simplified Human Visual Model

In general, an object $O(x, y)$ (for example, a picture on a graphical display) is degraded by convolution with the PSF of the user's visual system, $H(x, y)$, resulting in a distorted projection of the object on the user's retina, $I(x, y)$. This is described by

$$
I(x, y)=H(x, y) \circ O(x, y), \quad x=0, \ldots, N-1, y=0, \ldots . M-1
$$

where $\circ$ denotes convolution.

Given $O$ and $H$, the goal is to find an inverse function, $H^{1}$, to produce an enhanced object, $E O$, counteracting the distortion that will be introduced by $H$, such that when the user views the $E O$ on the graphic display, an undistorted version of $O$ will be projected onto the retina. This is described in equations 1-2 and 1-3.

$$
E O(x, y)=H^{-1}(x, y) \circ O(x, y)
$$




$$
I(x, y)=H(x, y) \circ E O(x, y)=H \circ H^{-1} \circ O(x, y) \approx O(x, y)
$$

This model amounts to a noiseless deconvolution problem. However the illconditioned nature of practical deconvolution [3] will require a more robust method to allow an efficient interaction between a user that has a refractive error present in his/her visual system and the PC. 


\section{CHAPTER 2}

\section{LITERATURE REVIEW}

2.1 Prior research in image enhancement for low vision

There has been some research done in the field of image enhancement for the visually impaired in order to improve face recognition, reading of text, and even graphical user interface design. As far back as 1981, high-contrast photographs were suggested as a visual aid to facilitate patient access to natural images [67]. Kenney reported on the use of large, high-contrast photographs to aid museum visitors that had visual impairments [64].

Peli and Peli proposed a conceptual pre-emphasis model of image enhancement that implies images may be processed before presentation to the patient to compensate for the degradation caused by the patient's visual dysfunction [61, 62]. Peli went on to further develop a linear pre-emphasis model, relying on knowledge of the contrast sensitivity function (CSF) of the patient [54]. He assumed that the CSF can represent the modulation transfer function (MTF) of the visual system. In his examples, the impairment studied was cataracts, which can be simulated as a linear filter. The ratio of a patient's CSF to a normal observer's CSF is called the Visual Degradation Transfer Function (VDTF) and is assumed to measure the optical transfer function of the cataractous lens.

$$
M T F(f)_{\text {cataract }}=\operatorname{VDTF}(f)=\frac{C S F(f)_{\text {patient }}}{\operatorname{CSF}(f)_{\text {normal }}}
$$

The inverse of this ratio, $1 / V D T F(f)$, may be used in the linear implementation of the pre-emphasis model [54]. This is shown in Figure 2-1. Using this method, Peli 
reported a statistically significant increase in recognition of face images using the preemphasis method versus nothing at all [57].
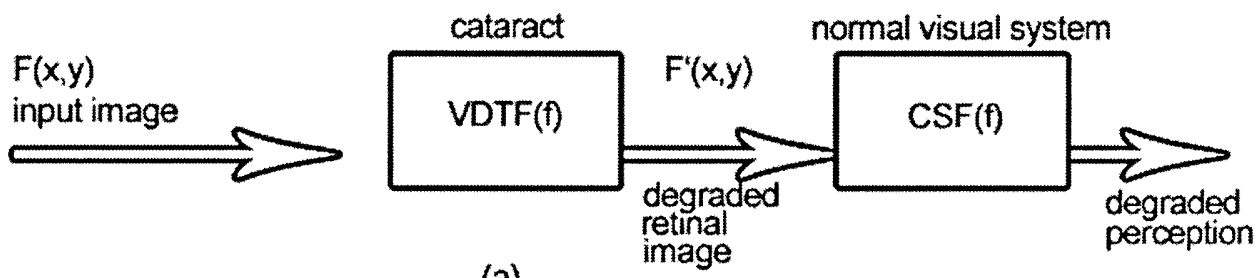

(a)

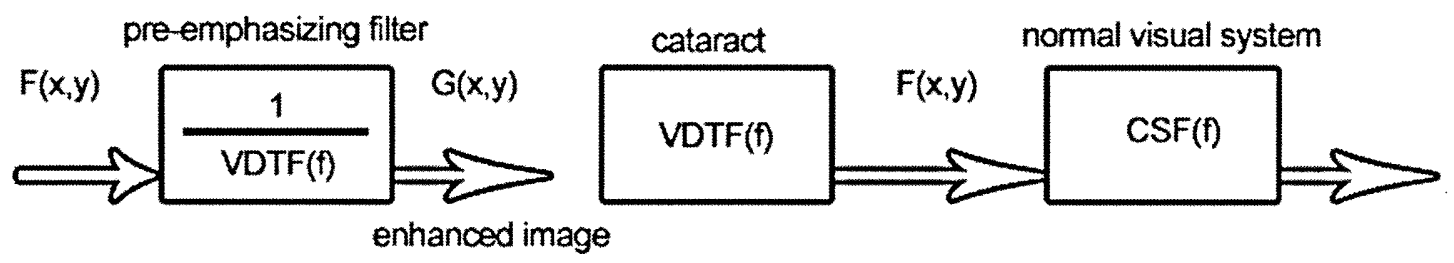

(b)

Figure 2-1 Linear Pre-emphasis Model [50]

Peli in a subsequent publication noted two major limitations of the method: the appearance of substantial high-frequency noise in the processed image, and the limitations imposed on the pre-emphasis enhancement by the finite dynamic range of the display device [50].

Lawton proposed two methods to reduce the high frequency components [58]. First, Lawton band-limited the images to frequencies within the visual range of the patients being testing. Second, a high-frequency noise reduction factor, A, was added to the pre-emphasis filter, $G(f)$ : 


$$
G(f)=\frac{\operatorname{VDTF}(f)}{\operatorname{VDFT}^{2}(f)+A}
$$

The best value for $A$ was determined empirically based on each patient's performance. These changes substantially increased the reading rate of three patients being studied $[56]$.

Peli and Peli [62] also proposed a method to address both problems via the application of an adaptive image enhancement algorithm [65]. When trying to enhance an image that occupies the full dynamic range of the display, the amplified high frequencies will exceed this range. They proposed that to provide the required dynamic range for the high frequencies, the local luminance levels or low frequencies must be modified as well.

The adaptive image enhancement algorithm achieves just that. It separates the low and high spatial frequency components. The low frequency component is obtained by filtering the image with a low pass filter, which has the effect of calculating the average brightness level found in a small window around each pixel. The high frequency component is obtained by subtracting the low frequency component from the original image. The high frequency component was then amplified. The AC portion of the low frequency component was attenuated by a factor of 0.9 permitting an additional range for the amplified high frequency component. The two components are then added together to produce the final image. This is shown in Figure 2-2. With this method, Peli reported a statistically significant improvement in face detection amongst subjects with central scotoma and macular disease [50]. 

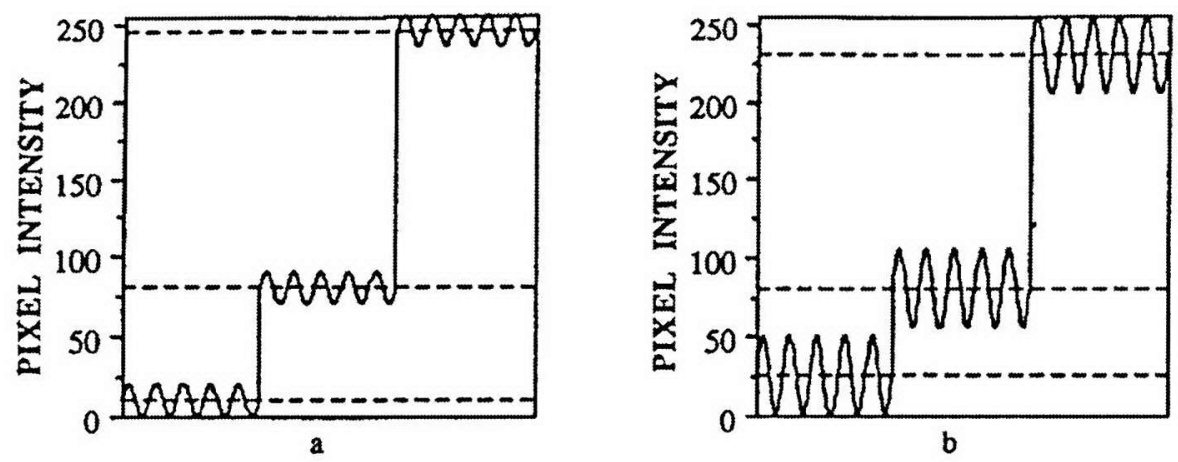

Figure 2-2 Extending the dynamic range in adaptive image enhancement [50]

Higuchi et al. proposed a way to simulate human visual capabilities of the elderly to aid the design of graphical user interfaces (GUIs) around these simulations [29, 34]. They chose to model three physiological properties of the human in their design of GUIs: adaptation to light, spatial sensitivity, and spectral sensitivity of the eye. They used empirical measurements of the physiological properties of the eye, as well as the display properties to establish the relationship between what is displayed and what is perceived by the user. These include a brightness function to estimate perceived brightness, a Gaussian low-pass filter, "tuned" to represent the spectral sensitivity of the observer, and a function that maps perceived color to age. They proposed this as a tool to aid designers in optimizing digital displays for users of various ages.

\subsection{Using Wavefront Analysis for Compensation of Visual Aberrations}

The methods mentioned above do not use an objective measure of the visual performance of the human eye. They rely on subjective observations from the user to determine parameters for the compensation process, such as the contrast sensitivity function (CSF) [50, 56]. Although effective for providing compensation in the types of 
impairment studied, they do not take into account the objective measurement of the optical aberrations in the human eye as part of their model.

Helmholtz was the first to observe that the human eye suffered from many aberrations that are not found in conventional optical systems [74]. There have been many methods developed to quantify these aberrations $[51,63,75]$. As recent as 1994 , a complete objective measurable descriptor for the human eye that was rapid and automated was unavailable to the public. However, in 1994, Liang et al. [45] developed a technique to measure the wave aberration of the human eye based on the ShackHartmann wavefront sensor [72]. Liang and Williams [39] further developed this method to measure the eye's aberration up to 10 radial orders.

Since then, commercially available instruments that objectively measure the wavefront aberrations have become common in ophthalmology $[8,13]$. These currently available "Wavefront Analyzers" can measure the wavefront aberration of human eyes and report them as series of Zernike polynomials, which through a series of transformations, yield the point spread function (PSF), a complete descriptor of the way an optical system maps an object onto the image plane [31,35].

The availability of an objective measurement of the wavefront aberration function of the human eye has provided the possibility of processing the images displayed to computer users with significant visual distortions (such as severe myopia and Keratoconus $[8,19])$ with the goal of effecting a customized precompensation of these images. 
2.3 Current methods of Compensating for the PSF

\subsubsection{Adaptive Optics}

In 1997, Liang et al. proposed a method to compensate for the aberrations in the human eye by means of adaptive optics [40]. Adaptive optics has mainly been used to correct the dynamic wave-front error that atmospheric turbulence causes in astronomical telescopes $[53,69]$. It was first proposed by Babcock in 1953, and has been successfully implemented to correct the wave-front in these instruments [76].

Liang and Williams further refined the technology and combined a HartmannShack wave-front sensor with a deformable mirror to correct the aberrations of the eye in the study of visual performance to provide supernormal image quality [40]. Figure 2-3 shows the schematic configuration of the adaptive optics system for the human eye developed by Liang and Williams. A super luminescent diode (790 $\mathrm{nm}$ wavelength) creates a point source on the retina. The reflected light from this retinal spot is delivered to the wavefront sensor where wave aberration of the eye is measured. The 97 -channel deformable mirror is then shaped to compensate for the eye's aberrations, resulting in a nearly planar wavefront.

Liang and Williams reported that they were able to compensate aberrations up to fourth order with their adaptive optics setup. The second order aberrations, defocus and astigmatism, as well as the higher order aberrations such as coma and spherical aberration were reduced by a factor of 4 [50]. 


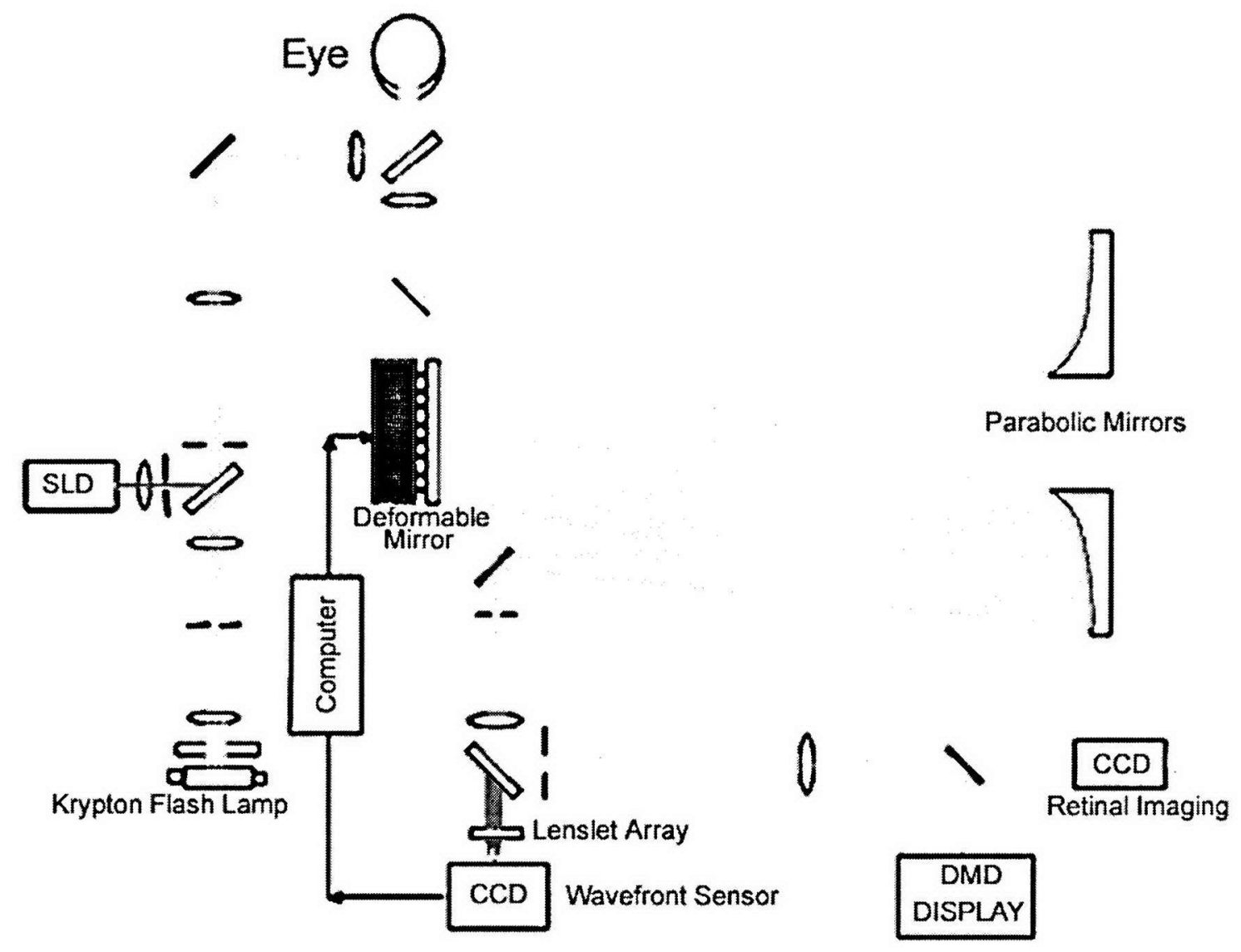

Figure 2-3 Adaptive optics wavefront compensation setup [6]

\subsubsection{Spatial Light Modulator}

Larry Thibos has explored the idea of compensating for the wavefront aberration error using a spatial light modulator as a means to counter the deviations of an aberrated wavefront from an ideal one. Thibos argues that spatial light-modulators (SLM) created from nematic liquid-crystals offer an attractive alternative to the technology developed by Liang and Williams in adaptive optical systems intended for use with the eye [41].

A spatial light modulator (SLM) is an electro-optical component used to control the phase or amplitude of a light waveform independently at different spatial locations 
across a two-dimensional plane. A liquid crystal SLM consists of a thin layer of liquid crystal molecules compressed between two parallel glass plates [52].

The glass plates are subdivided into a honeycomb array of control cells which divides an incident wavefront into segments, allowing spatial control. Each independent control cell contains liquid crystal molecules oriented in parallel (as in a crystal), which causes the cell to be birefringent. Consequently, when light polarized in the direction parallel to the molecules passes through the crystal, it is subjected to a different refractive index than light polarized in a direction orthogonal to the molecules. The molecules are made to rotate when an electric field of increasing strength is applied to transparent conductive electrodes coated on the inside of the glass plates. Thus, an applied voltage can be used to vary the refractive index experienced by light of fixed polarization when transmitted through the crystal [41].

Since the liquid crystal SLM is an electronically controlled array of transparent cells of variable refractive index, it has the potential for producing computer-controlled optical elements, as well as for correcting not just the sphero-cylinder refractive errors of the eye but more complex, higher-order optical aberrations as well [41].

Because the wavelength of light in the medium of the crystal is inversely proportional to the material's refractive index, the wavefront is retarded relative to the same wavefront propagating in air. Consequently the wavefront emerges with a different slope, propagating in a different direction. Figure 2-4 illustrates the alternative principle by which the SLM refracts light. A wavefront propagating from left to right (dashed 
lines) passing through a medium of higher refractive index (shaded region). It is retarded, causing the direction of propagation (arrows) to change [41].

Figure 2-5 shows the apparatus setup used by Thibos in testing an SLM compensator. The observer viewed a Bailey-Lovie eye chart [71] that was projected on a screen with a high-intensity projection monitor (Proxima 5900). A lens collimated light from the screen for transmission through an interference filter, a polarizing filter, the SLM, and a $12 \mathrm{~mm}$ diameter aperture to mask the active area in the SLM. A pair of relay lenses imaged the SLM in the eye's pupil plane with a magnification factor of 0.25 . This reduced the image of the SLM formed in the eye's entrance pupil to $3 \mathrm{~mm}$ in diameter [36].

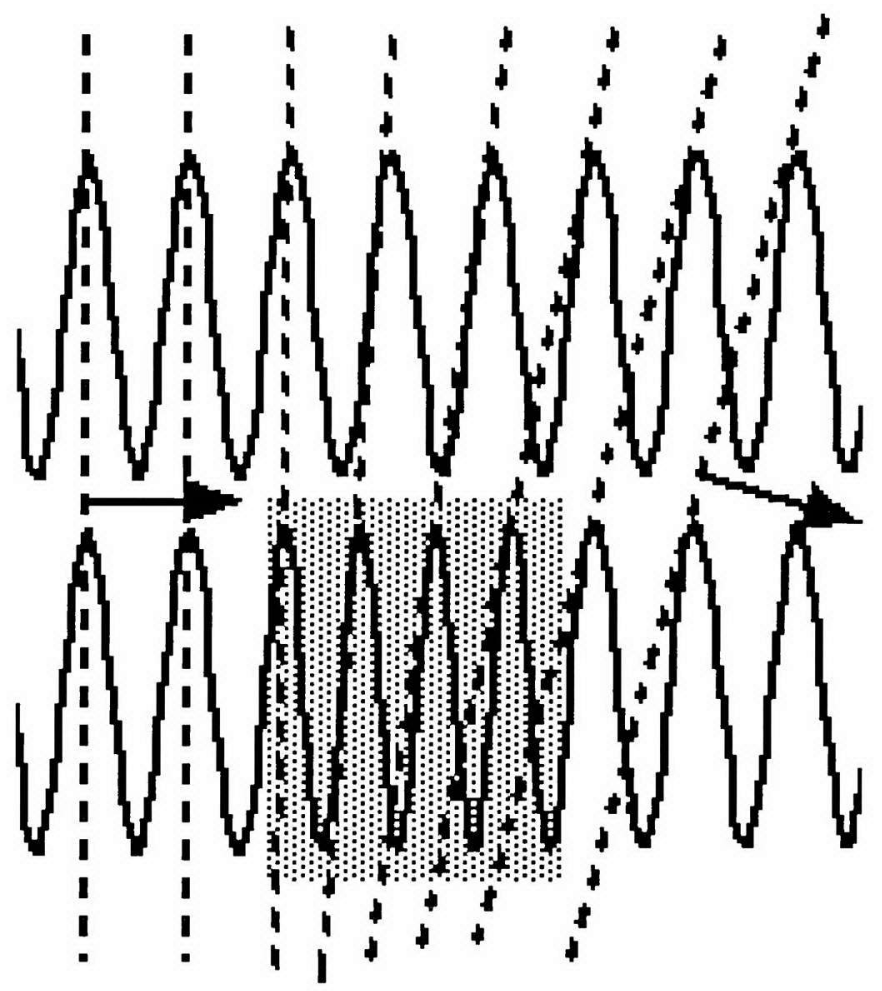

Figure 2-4 Principle of refraction by a spatial light modulator (SLM) [41] 


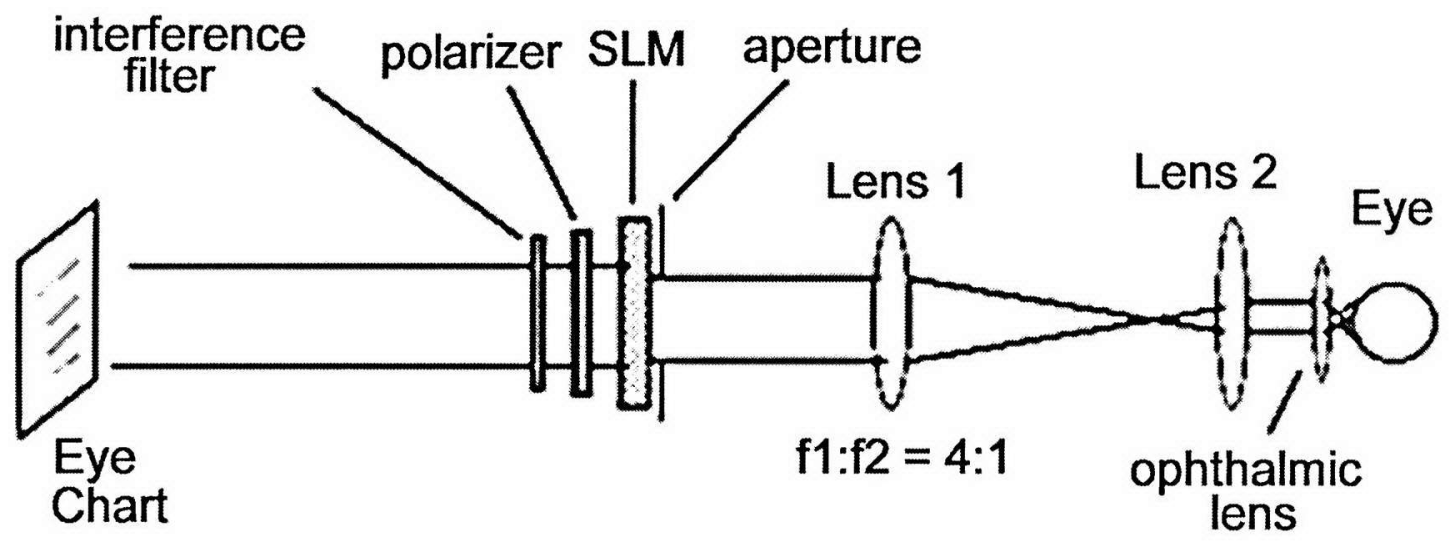

Figure 2-5 SLM Compensation Apparatus Setup [36]

Thibos reported that the SLM effectively neutralizes the refractive error induced by the ophthalmic lens provided that the lens power is no larger than 1.5 Diopters. The acuity for the subjects tested remained near the normal level of 20/20 (6/6 metric) when the refractive error induced by the ophthalmic lens was corrected by the SLM. However, when the induced refractive error exceeded 1.5 Diopters, acuity fell rapidly.

\subsubsection{Software Precompensation}

Alonso et al. [3] proposed an alternative to hardware-based compensation of the wavefront aberration of the human eye. It is a software-based method that "precompensates" images based the PSF of the human eye through deconvolution based on the principles of Fourier optics. The rationale for this alternative approach is that hardware is both costly and space inefficient, and with the increased number of computer users, a software based method of precompensation of graphical user interfaces (GUI) would be more practical and useful in addressing wavefront aberrations in the human eye. Figure 2-6 shows a block diagram of the proposed software pre-compensation system. 


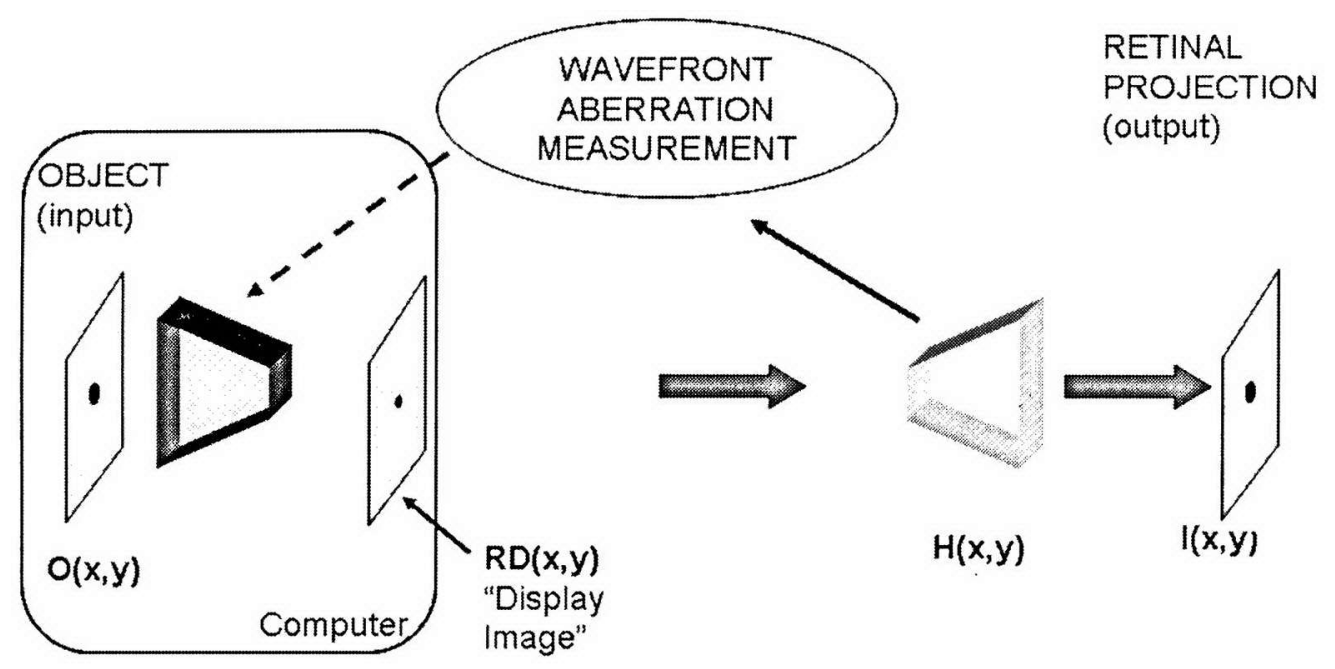

Figure 2-6 Software Pre-compensation process

The display image $\mathrm{RD}(\mathrm{x}, \mathrm{y})$ is the result of deconvolving the user's PSF from an object image, $\mathrm{O}(\mathrm{x}, \mathrm{y})$, using a straight application of the Weiner Filter[17]. When viewed through the PSF, the distortion is countered and the result is a clearer projection of the object image. In order to overcome the loss of contrast, adaptive histogram equalization was used in [3] to increase the contrast of the precompensated object. Alonso first tested this idea by using a generic blur PSF to simulate the blur introduced by defocusing a digital camera. The camera was then used as the "wavefront aberration" for 14 human subjects (28 human eyes) and the visual acuity was measured for each eye.

Alonso found a statistically significant increase in visual acuity observed through the artificial blur when viewing images that were precompensated versus images that were not. This results in 50\% improvement in visual efficiency when viewing the precompensated objects [3]. 
This dissertation seeks to extend the work done by Alonso by introducing new concepts in deconvolution and spatial processing to improve the quality of the precompensation process. Additionally, the work done by Alonso is also extended by using the PSFs of real subjects in the pre-compensation process, as well as testing with these human subjects and observing the improvements in visual acuity by means of standard eye tests and computer related tasks. 


\section{CHAPTER 3}

\section{METHODS}

\subsection{Human Vision}

\subsubsection{Physiology of the Human Eye}

The optical components of a typical adult human eye are shown in Figure 3-1 as an anatomical cross section.

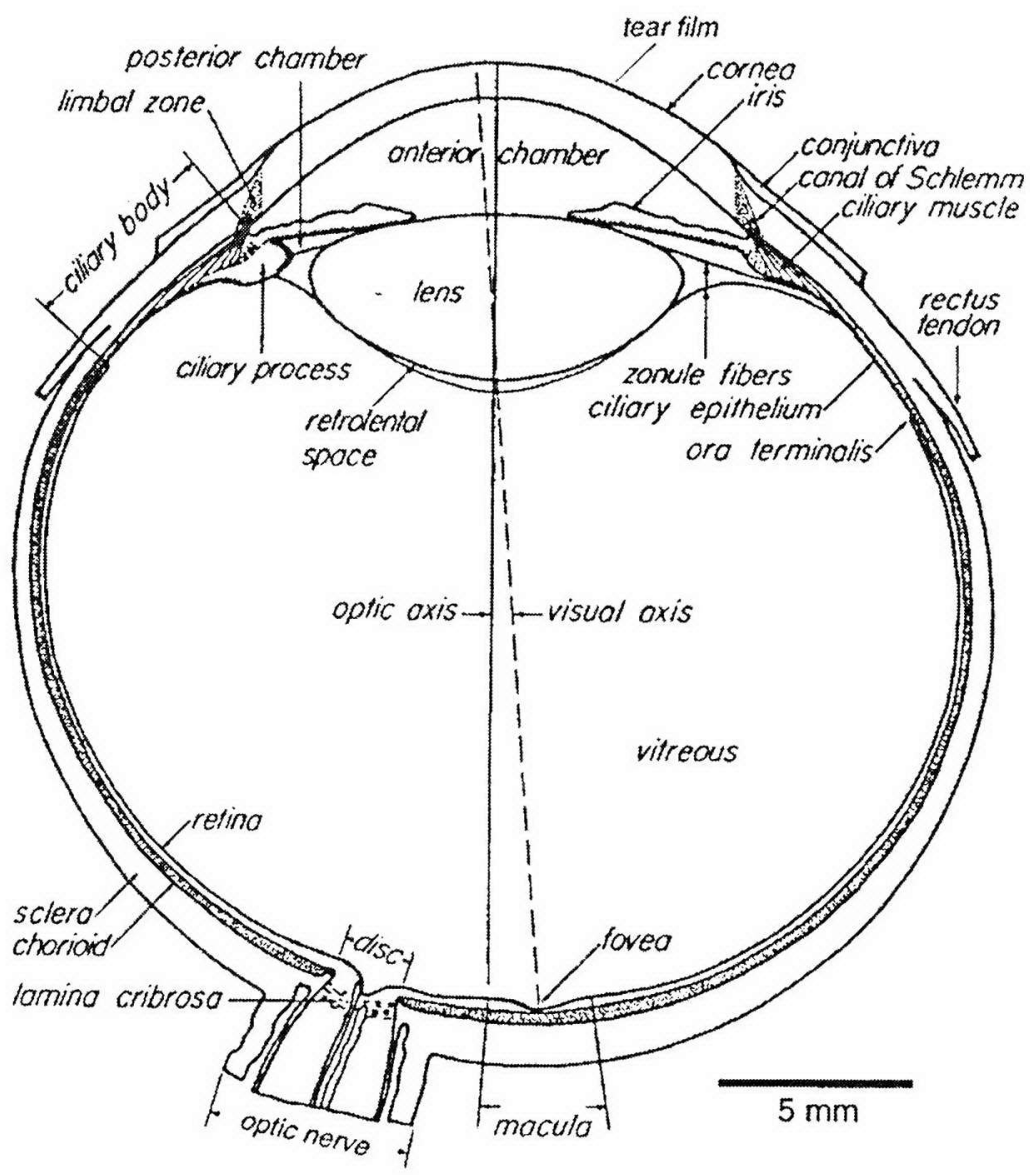

Figure 3-1 Anatomical view of the human eye [31]

The human eye is equivalent to a digital camera, consisting of a lens, a dynamic aperture system (the pupil), and the retina [42] (which can be thought of as equivalent to the charge-coupled device (CCD) in a typical digital camera). The lens system of the eye 
is composed of four refractive surfaces, each with a distinct refractive index; $n$. Light arrives at the cornea (a thin, translucent membrane) and is refracted or redirected due to a change in its speed. The cornea provides most of the refractive power of the eye, having an index of refraction of approximately 1.38 . Light then travels into the aqueous humor $(n=1.33)$ where it is once again refracted before it reaches the crystalline lens $(n=1.4)$. Because the crystalline lens is surrounded by fluid on both sides (the aqueous and vitreous humors), it only accounts for about $1 / 3$ of the total refractive power of the eye. Light finally exits from the lens into the vitreous humor $(n=1.34)$, once again being refracted before it reaches the retina. [42]

If all of the refracting surfaces of the eye were collapsed into a single lens, it would have a total refractive power of approximately 59 Diopters (or equivalently a lens with a focal length of $17 \mathrm{~mm})[42]$.

\subsubsection{The Eye as a Dynamic Optical System}

If the eye were a static optical system, human beings would only be able to see clearly under very specific conditions, such as under a certain amount of illumination and at a certain distance. There are two main optical mechanisms that allow humans to see objects in the world under various viewing conditions: accommodation and pupil diameter.

Accommodation is the eye's ability to change the refractive power of the lens. The crystalline lens, located behind the iris, is responsible for the eye's ability to dynamically change its refractive state, providing a mechanism for focusing at various distances [55]. In children and young adults, the internal lens of the eye is flexible 
enough that its anterior surface can bulge forward to increase the optical power. This change in shape occurs as tension on the supporting zonule fibers is released in response to forward movement of the ciliary processes caused by contraction of the ciliary muscle $[37,77]$.

The iris of the human eye acts as an aperture that controls the amount of light that enters the eye, and is proportional to the area of the pupil [42]. The pupil has a range of diameters of $1.5 \mathrm{~mm}$ to about $8 \mathrm{~mm}$, which is roughly a ratio of 28 to 1 , that is, changes in the pupil diameter from $1.5 \mathrm{~mm}$ to $8 \mathrm{~mm}$ allow approximately 28 times more light to enter the eye [42].

Given the autonomous variability of both the accommodative state of the eye, as well as the pupil diameter, the optical characteristics of the human eye are constantly changing. Current technology to characterize the optics in the human eye does so under certain viewing conditions, namely a relaxed accommodative state. Any divergence in viewing conditions from those used to characterize the eye will change the characterization $[7,12]$. For example, the eyes of a person viewing a liquid crystal display (LCD) panel will not be in a relaxed state due the fact that he/she must accommodate for viewing the LCD panel at a finite distance.

Additionally, luminance conditions may vary from those originally used to obtain the characterization, thus causing a change in the pupil diameter [12]. There are two major sources of aberrations in the human eye, both relating to the size of the pupil. For pupil diameters of $2 \mathrm{~mm}$ or less, the major contribution to distortion of the retinal image is due to diffraction. For pupils larger than $2 \mathrm{~mm}$, the aberrations of the optical system 
distort the image formed on the retina. The wave theory of image formation accounts for both of these potential sources of distortion $[31,73]$.

\subsubsection{Mathematical Model of the Human eye}

Ideally, the human eye focuses all of the rays of light from a distant point entering the eye into a single image point on the retina (Figure 3-2). Paraxial optics can predict where along the optical axis this point will be. But in the real world, the eye is not a perfect optical instrument and has three types of optical imperfections which are not addressed by paraxial theory: aberrations, diffraction, and scattering [31]. Even though the mechanisms of the imperfection are different in each case, the overall effect of these imperfections is to spread light across the retina, as shown in Figure 3-2(b). Additionally, the spatial distribution of light intensity in the image can be understood with the concepts and computational tools of Fourier analysis [73].

a

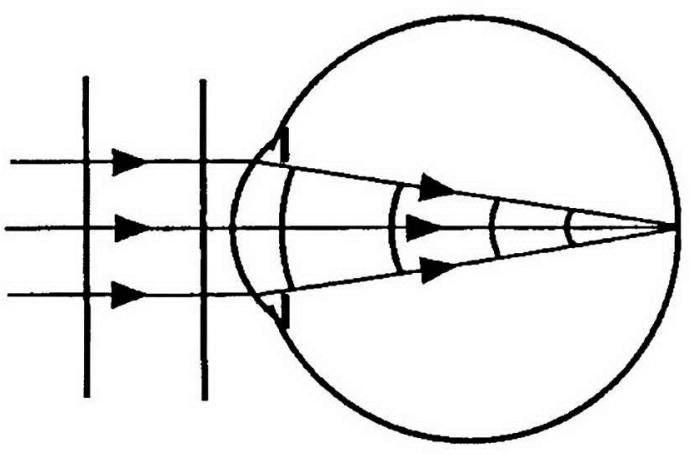

b

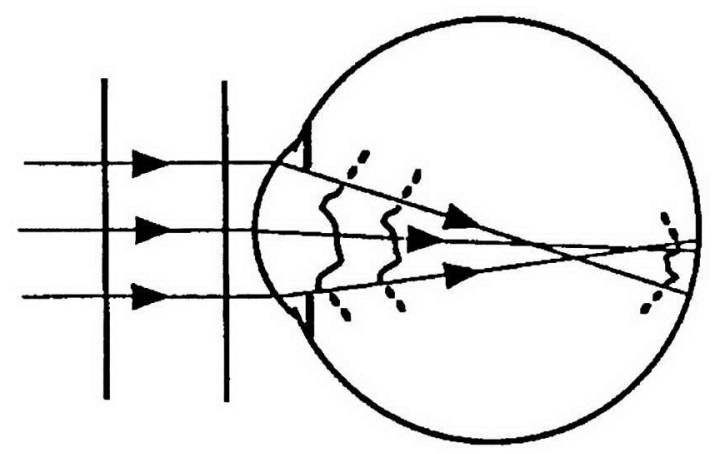

Figure 3-2 Light spread on the retina by (a) an ideal eye and (b) an aberrated eye [31]

When light passes through an optical system that contains aberrations, it will exit from the pupil as a wavefront that is aberrated from the ideal spherical surface, known as the 'pupil sphere'. Once the wavefront aberration function is known, the principles of 
Fourier optics can be used to derive the optical transfer function (OTF) and the point spread function (PSF) of the optical system. The OTF and the PSF are a Fourier transform pair, which is summarized in Equation 3-1.

$$
\begin{aligned}
& O T F(f x, f y)=F\{P S F(x, y)\} \\
& I(x, y)=P S F(x, y) \circ O(x, y)
\end{aligned}
$$

Most of the effort aimed at defining the PSF of the eye has been directed towards measuring the wavefront aberration function [31] which is usually reported as a set of Zernike coefficients $[27,32]$. From this set of Zernike polynomials, the PSF can be derived and thus provide a complete description of the performance of an optical system (much like its one-dimensional counterpart, the impulse response). The output of this optical system is therefore described by a convolution of the input to the system with the PSF. This is shown is Equation 3-2, where $O(x, y)$ is the object that is to be imaged onto the retina, $\operatorname{PSF}(x, y)$ is the point spread function of the eye, and $I(x, y)$ is the retinal image. Appendix A contains a detailed description of how to obtain the PSF of an optical system from its wavefront aberration function described by a set of Zernike polynomials.

\subsection{Pre-compensation System}

\subsubsection{Spectral Processing for Inverse Solution}

Once the PSF is obtained from the wavefront aberration of each user, the first step in realizing a display system that will provide compensation for the aberrations in the refractive portion of the user's visual system is to inverse-filter the image to be displayed using the PSF. Recall Figure 1-1 which describes the linear systems view of imaging in the human eye. The image that is to be shown to the user will be referred to as the object, 
$O(x, y)$, the retinal image will be referred to as the image, $I(x, y)$, and the point spread function will be referred to as the $\operatorname{PSF}(x, y)[1,3,31,35]$. Thus, the image and the object are related through convolution (denoted by 0 ), as shown in Equation 3-3:

$$
I(x, y)=O(x, y) \circ P S F(x, y)
$$

The goal of the inverse filtering process is to produce a precompensated object using the model of the user's visual system. The objective is that when the user views this precompensated object, it will be projected with little distortion onto the retina. It is well known that spatial convolution, as in Equation 3-3, is equivalent to multiplication in the frequency domain, that is,

$$
I(f x, f y)=O(f x, f y) \cdot O T F(f x, f y)
$$

From equation 3-4, the frequency domain representation of the precompensated object given by the deconvolution of the PSF from the object, is

$$
O_{P C}(f x, f y)=\frac{O(f x, f y)}{O T F(f x, f y)}
$$

Although, Equation 3-5 gives a mathematically accurate precompensated object, it is very sensitive to small values in the OTF [59]. Figure 3-3 shows the image of an icon and its resulting inverse produced by using Equation 3-5, employing an example defocus PSF.

A technique is employed to control the singularities of the inverse. This is called regularization [70]. In order to regularize the inverse transfer function, a parameter $\varphi$ is introduced (Equation 3-6) that controls the level of accuracy of the deconvolution as well 
as the sensitivity of the inverse $[17,21]$. This is subjectively chosen to achieve a desired level of tradeoff between the accuracy of the inverse and the loss of contrast as a result of displaying these images on a real LCD panel. Further discussion on the practical display of precompensated objects is presented in section 3-3.

$$
O_{P C}(f x, f y)=\frac{O(f x, f y) \cdot O T F^{*}(f x, f y)}{|O T F(f x, f y)|^{2}+\varphi} \cdot \frac{1}{O T F(f x, f y)}
$$
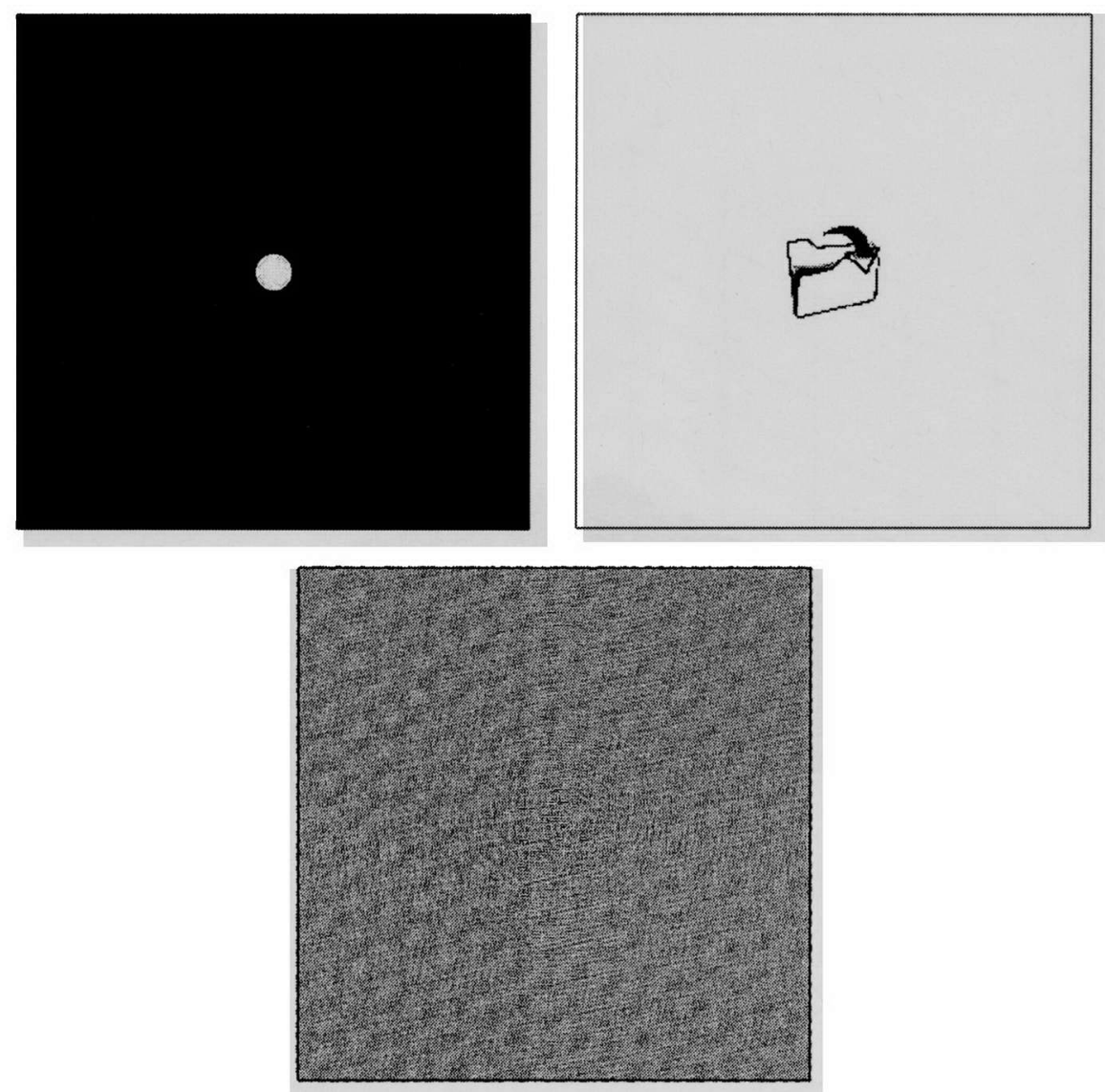

Figure 3-3 Example of ideal inverse filter: PSF (top left) Object (top right) Precompensated Object (Scaled for Display, bottom)

Equation 3-6 already provides a precompensated object $O_{p c}(x, y)$. However, in addition to deconvolving the known PSF, this processing introduces artifacts in the form 
of "ripples" in the precompensated object [1]. Furthermore, the numerical values of $O_{p c}(x, y)$, found mathematically by Equation 3-6, may not be constrained to the range of available grayscale levels of $[0,255]$ for modern LCD panel displays. The artifacts introduced by using equation 3-6 on an example image using a defocus PSF, as well as the resulting amplitudes exceeding the displayable range are shown in Figure 3-4 and Figure 3-5. Figure 3-6 shows the entire process.

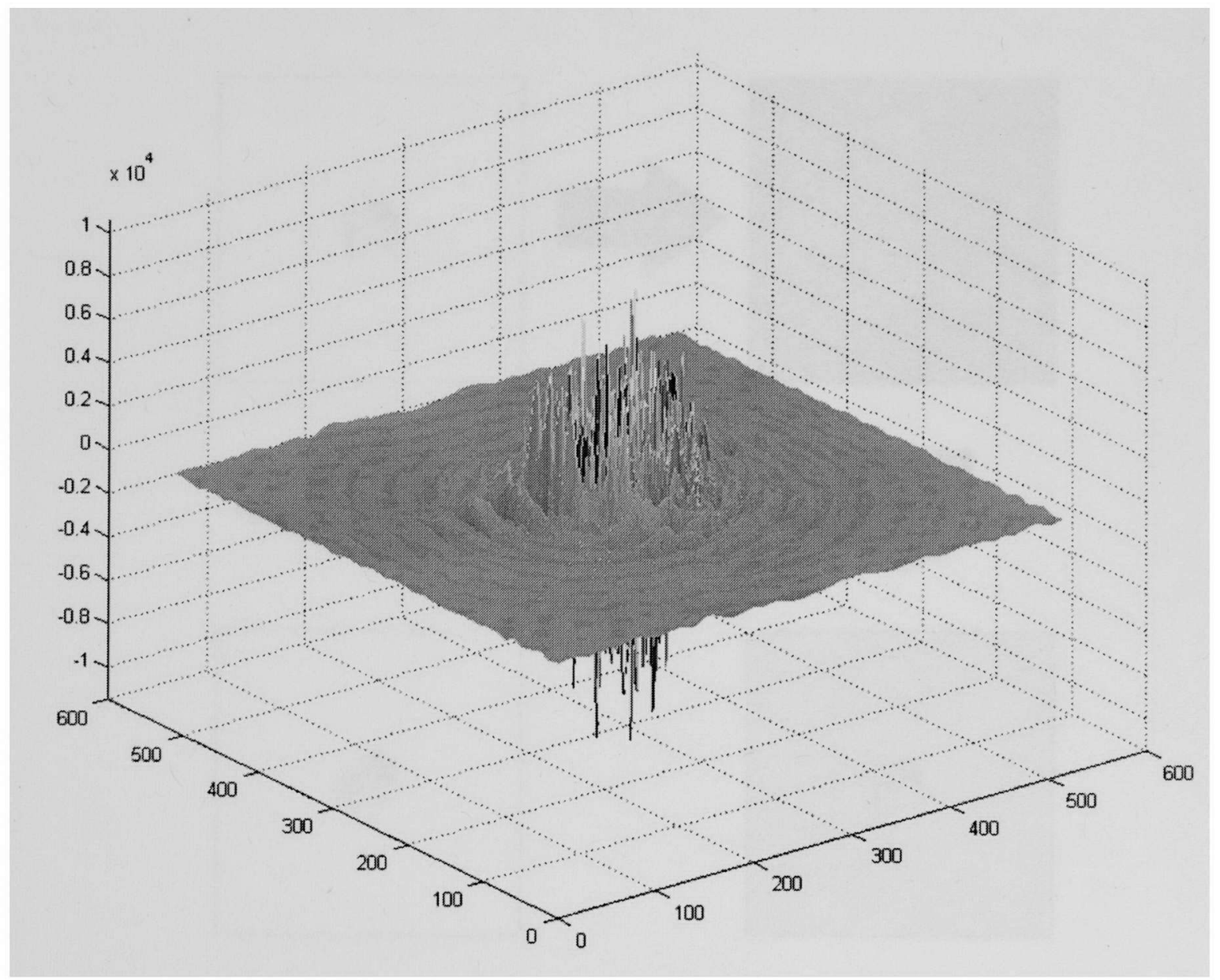

Figure 3-4 Example of Pre-compensated Folder Icon 


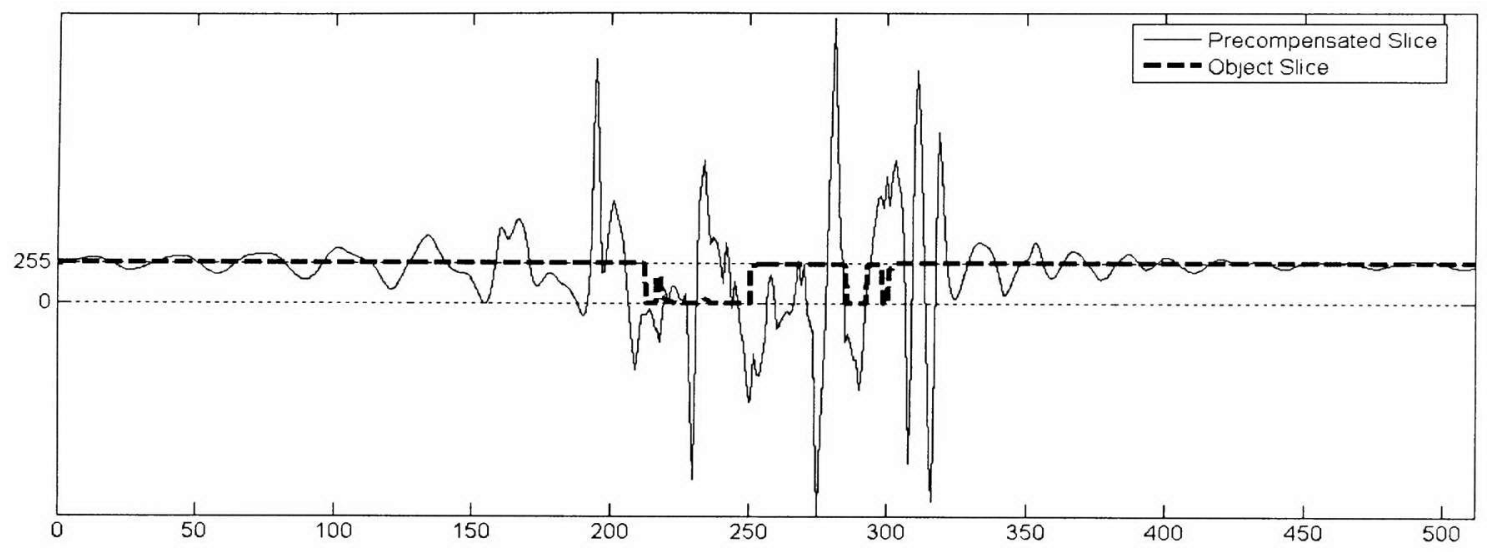

Figure 3-5 Bipolar result of the pre-compensation process (1D slice)
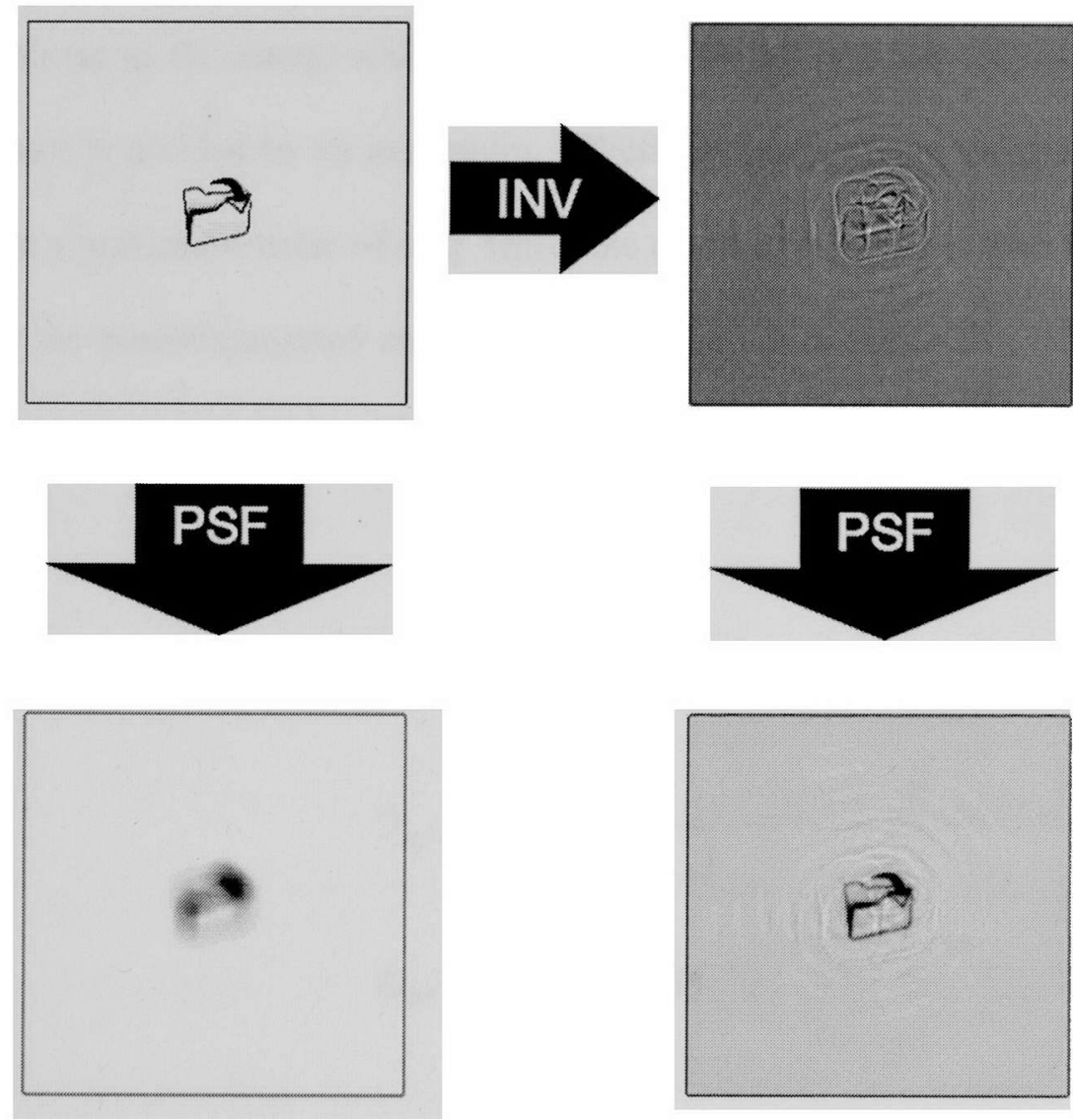

Figure 3-6 Simulation of ideal precompensation process: Icon with blur simulated (bottom left)

Precompensated Icon with blur simulated (bottom right) (Images are scaled for display) 


\subsubsection{Spatial Processing for Feature Extraction}

The ripples that are produced as a result of using Equation 3-6 are a roadblock for further processing of the precompensated object. Additionally, since display devices such as LCD panels can only represent saturation levels between 0 (no saturation) and 255 (full saturation) in any one of the three color bands (red, green, or blue, RGB), the precompensated object must be scaled and shifted from a bipolar range, to a unipolar range for display. This scaling can be accomplished in three steps: first by subtracting the minimum value of the precompensated object from the image itself. This retains the relative variations in the image while raising it to have all positive values. Second, the preceding result is divided by its maximum, effectively normalizing the precompensated object to have a maximum value of one. Third, the result of step two is then multiplied by 255 to allow the precompensated object to occupy the entire range of available display values. This process is summarized by the following equations:

$$
\begin{gathered}
O_{P C-A}=O_{P C}-\min \left[O_{P C}\right] \\
O_{P C-B}=\frac{O_{P C-A}}{\max \left[O_{P C-A}\right]} \\
O_{P C-D}=O_{P C-B} \cdot 255
\end{gathered}
$$

where $O_{P C-D}$ is the "shifted and scaled" precompensated object. Figure 3-7 shows a shifted and scaled precompensated object. 
(a)

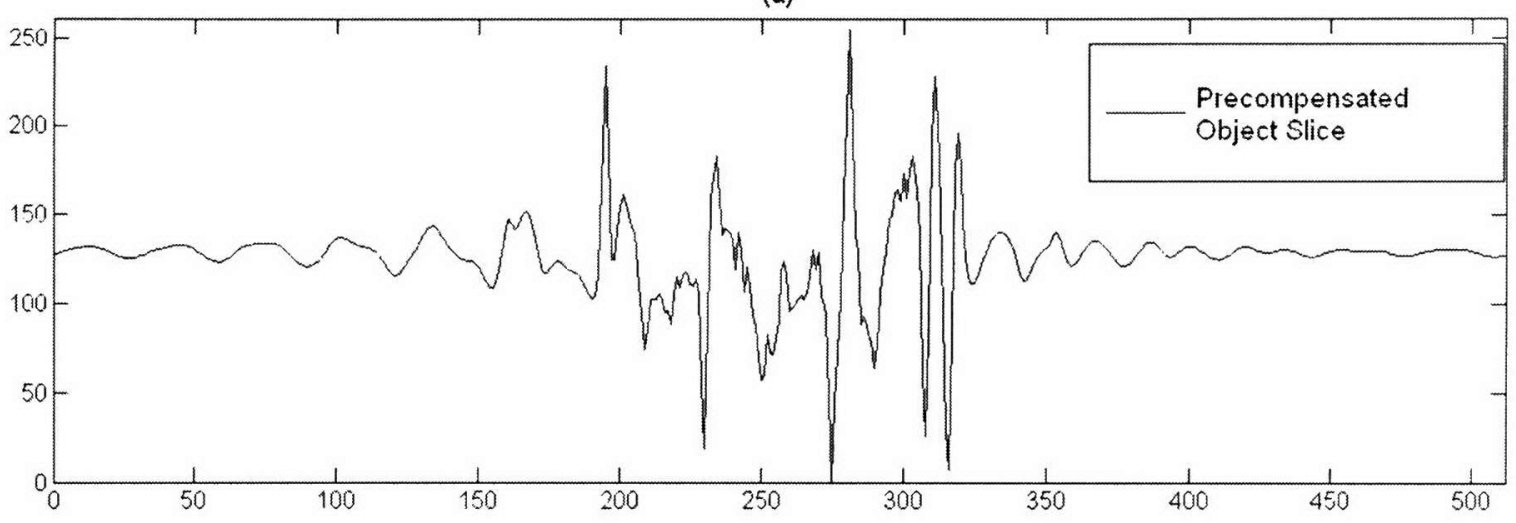

(b)

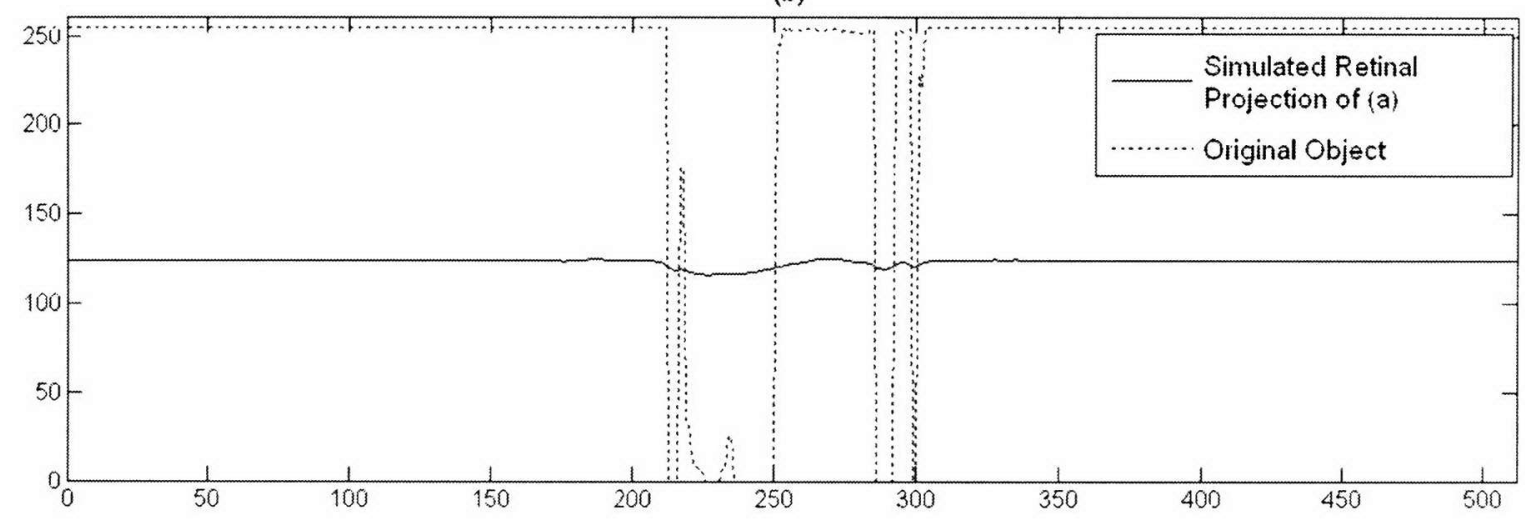

Figure 3-7 (a) Shifted and Scaled Precompensated object slice (b) Retinal Simulation with Object superimposed

This process of scaling and shifting reduces the available contrast in the resulting retinal projection making post-processing of the output obtained from the deconvolution stage necessary [3]. It must, however, be implemented in a way that does not exacerbate the negative impact of residual ripples introduced by the deconvolution. Figure 3-7(b) shows the result of simulating the retinal projection of the precompensated object. There is a substantial loss of contrast when compared to the original object.

If, for example, histogram equalization is used for post-processing, it will improve the contrast in the precompensated object (which will also enhance the contrast in the 
image perceived by the user), but it will disproportionately amplify any ripples outside of the area of interest. These amplified ripples will then unduly compete for the viewer's attention, limiting the effectiveness of the precompensation. An example of this is shown in Figure 3-8.

(a)

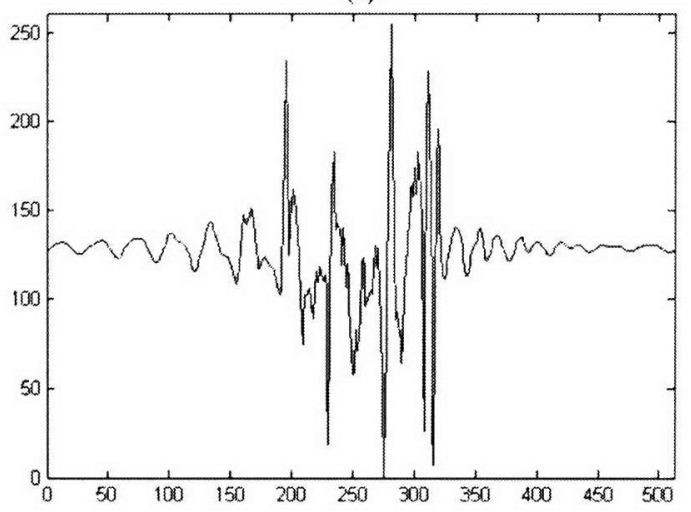

(b)

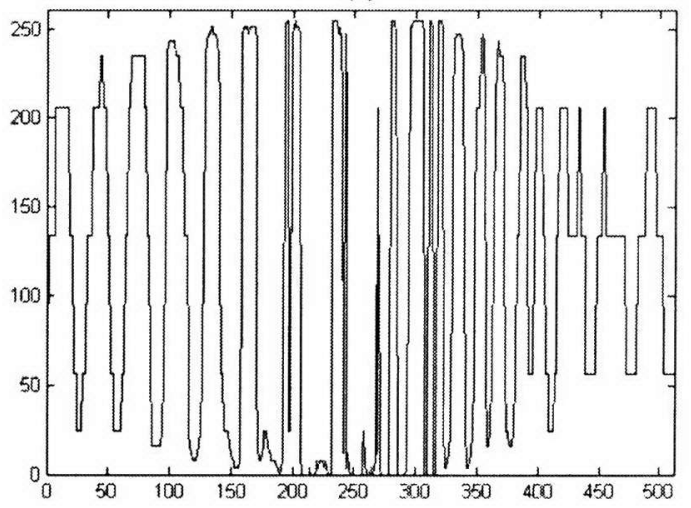

(c)

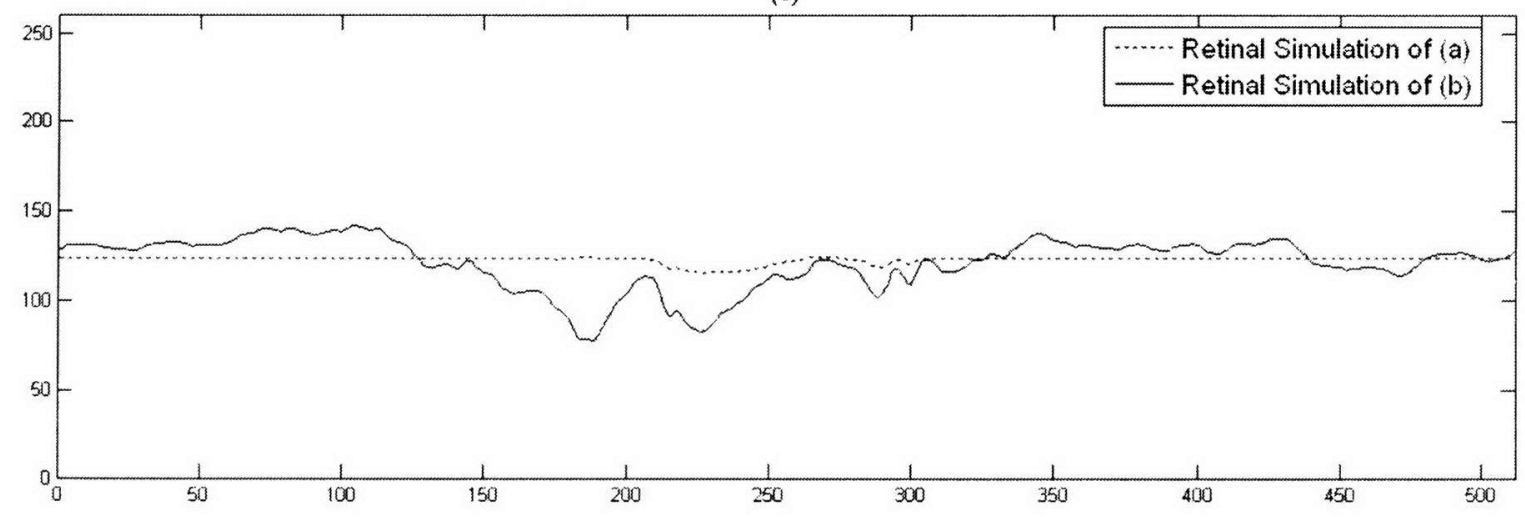

Figure 3-8 (a) The shifted and scaled precompensated object (b) histogram equalization of (a) (c) comparison of retinal simulation of (a) and (b)

I propose a method to extract only the necessary information from the precompensated object, so that the post processing contrast enhancement may correctly enhance the image. In this dissertation, text or icon images are considered digital images, composed of letters or icons on solid backgrounds. This subset of images is used because 
digitally generated images, such as icons, have very high contrast as compared to real images. This allows the full use of the dynamic range available in the LCD displays. Additionally, icons are chosen because they are inherent in the use of Graphical User Interfaces (GUIs) [30]. The ability to recognize and interact with icons is cornerstone in Human-Computer Interface problems. Since one of the primary goals of this dissertation is to provide adequate precompensation in order to improve computer interface of users with refractive errors, icons are a suitable image choice.

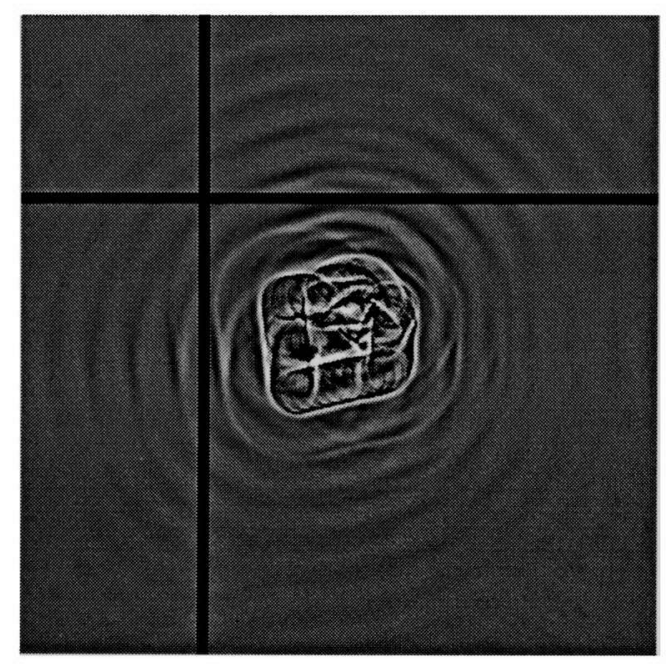

(a)

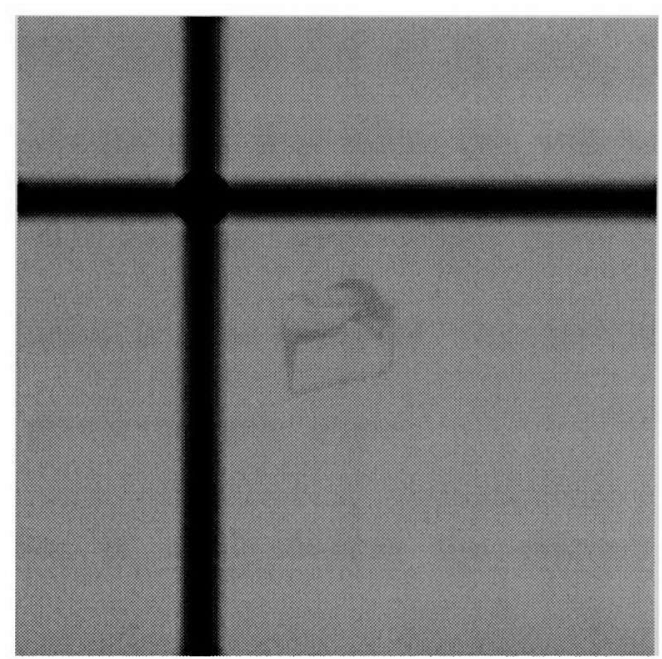

(b)

Figure 3-9 Not all of the information is necessary for reconstruction

When these objects are precompensated, only a finite region around the boundary of the letter or icon is necessary to yield its correct perception by the viewer. This is shown in Figure 3-9, where black bars have been inserted across the precompensated object and the blurring introduced by viewing through a defocus PSF has been simulated. The bars remove information from the precompensated object; however, this information is not necessary for correct perception of the precompensated object. The resulting 
simulated retinal image is still clear, showing that not all of the information in the precompensated object is necessary for a good reconstruction.

It is thus necessary to find only the areas of the precompensated object that must be present in order for a good reconstruction. A summary of this procedure is as follows. The first step is to segment the object, which isolates the areas of interest from the background. This will, when combined with subsequent processing, allow for the extraction of only the necessary information from the precompensated object. The result of the segmentation process is subsequently blurred using the PSF of the user. A threshold is applied to the blurred, segmented object (in this dissertation this will be either an icon or a letter) to produce a mask that contains zeros where there is unnecessary information and ones where the information is to be extracted. The mask is then used in a point-to-point multiplication with the precompensated object.

As mentioned above, image segmentation is used identify the relevant information in the digital image. The image segmentation process is summarized as follows. The first step in image segmenting is to find the edges. In order to do this, the Robert's cross-gradient operators ([8]) were chosen because their implementation is computationally efficient and provide a simple way to compute the first derivatives of an image. The first derivative is commonly used to find edges in an image [17]. The kernels used are shown in Figure 3-10.

Because the icon or text objects being used are noise-free, the Roberts crossgradient operators are sufficient to provide an accurate approximation of the gradient. 
The kernels shown in Figure 3-10 are used to approximate the gradient by means of a two-dimensional filter [17].
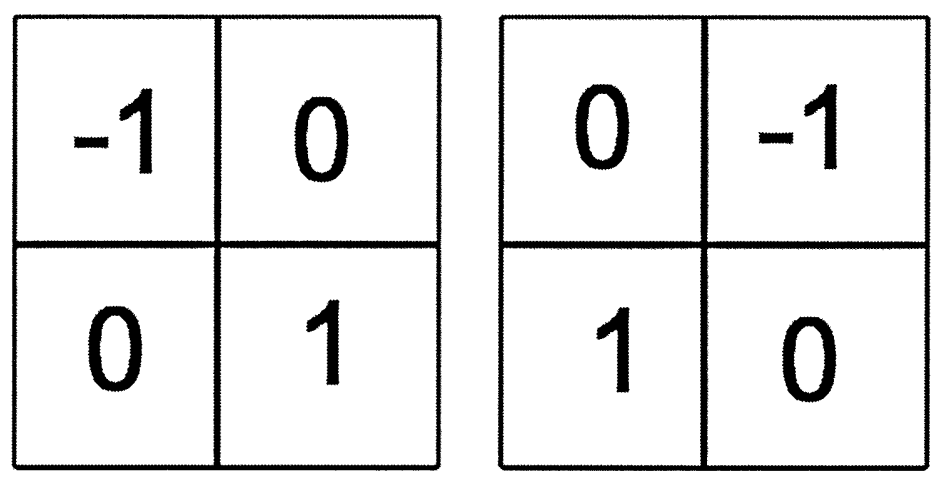

Figure 3-10 Robert's cross gradient operators

This filtering is implemented as a convolution of the icon objects with each one of the gradient operators:

$$
G_{1}(x, y)=O(x, y) \circ R_{1}(x, y),
$$

and

$$
G_{2}(x, y)=O(x, y) \circ R_{2}(x, y),
$$

where

$$
\begin{aligned}
& R_{1}(x, y)=\left[\begin{array}{cc}
-1 & 0 \\
0 & 1
\end{array}\right], \\
& R_{2}(x, y)=\left[\begin{array}{cc}
0 & -1 \\
1 & 0
\end{array}\right],
\end{aligned}
$$

$G_{I}(x, y)$ is the approximation to the gradient for the first Robert's operator and $G_{2}(x, y)$ is the approximation to the gradient for the second Robert's operator. The final approximation to the gradient is then given by 


$$
G(x, y)=\left[\left[G_{1}(x, y)\right]^{2}+\left[G_{2}(x, y)\right]^{2}\right]^{\frac{1}{2}},
$$

where $G(x, y)$ is the approximation of the gradient for the object $O(x, y)$.

Once the gradient is computed, a threshold is applied to the gradient to isolate only the strongest edges present in the object. This threshold is determined automatically by taking the square root of a factor $n$ times the mean of the gradient, where $n$ is chosen based on the amount of noise present in the image. The threshold is then given by

$$
t=\sqrt{n \cdot \mu_{G}}
$$

where $t$ is the value of the threshold, $n$ is the ad-hoc factor that is chosen depending on the noise level, and $\mu_{G}$ is the mean of the approximated gradient. For this dissertation, $n$ is held constant at a value of 6 , which yields good edge detection for the class of images used. Figure 3-11 shows an example of the edge detection method on the icon shown in Figure 3-3 (top right).

The edge detection process reveals spatial information about the icon or text with respect to its extent within the border of the object. This information is useful in determining the degree to which the PSF will affect the icon or text. One must take into account that the images used in this dissertation are ideal images in that they do not contain noise and occupy a small region of the entire area to be processed. Thus, with this edge information, the spatial extent (i.e. the extent measured radially from the center of the icon out) to which information in the precompensated object necessary in the reconstruction of the retinal image can be determined. 


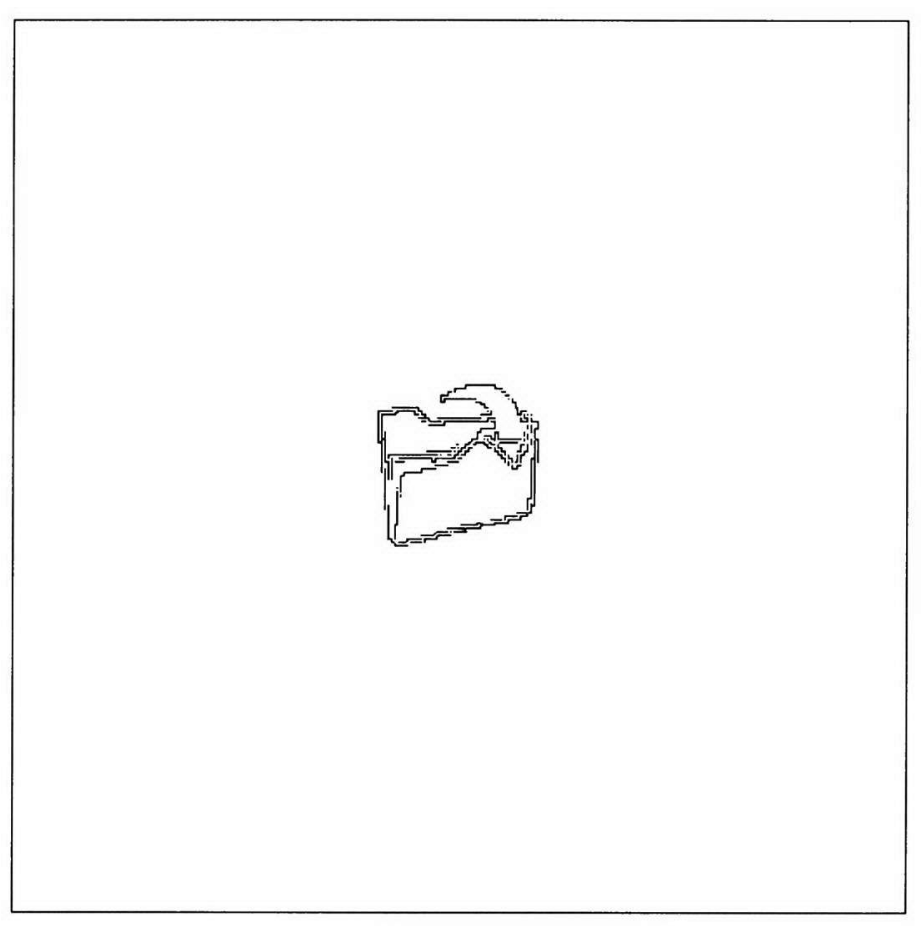

Figure 3-11 Edge detected icon shown in Figure 3-3

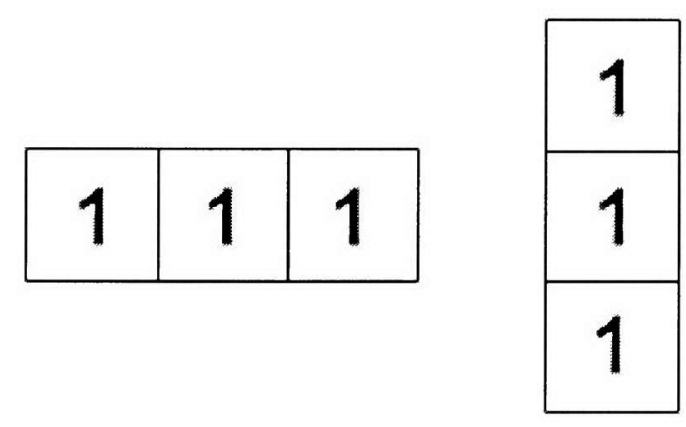

Figure 3-12 Horizontal (left) and Vertical (right) structuring elements

In order to define the strongest boundaries of the object and close any gaps may exist in the edge detected object, it is dilated using first a horizontal structuring element, and then a vertical structuring element. Applying this process to the edge detected object ensures that the strongest edges are connected, which is essential in order to "fill" the object in. The structuring elements used are 3 pixels long, oriented in horizontal and vertical direction. An example of them is shown in Figure 3-12. 
Dilation is a morphological operation and is defined as follows: "The dilation of A by B (denoted by $A \oplus B$ ) is the set of all displacements, z, such that B and A overlap by at least one element." [17] This will close any gaps that may exist in the edge-detected image (Figure 3-14c) and is written as [17]:

$$
A \oplus B=\left\{z \mid\left[(B)_{z} \cap A\right] \subseteq A\right\}
$$

The output of Equation 3-16 is a binary image containing ones where the edges of the icon are strongest, and zeros otherwise.

Once the object has been edge detected as described above, the next step is to fill in the interior areas bound by the outer edges of the icon. This is done by filling 'holes' in the binary image edge detected image. There exist several methods of accomplishing this $[60,66]$. However, a very simple algorithm for marker based region filling is given in [17]. If all non-boundary points are assigned to be ' 0 ', and boundary points are assigned to be ' 1 ', then an iterative method of filling the 'holes' beginning from a starting marker point, $p$, is

$$
X_{k}=\left(X_{k-1} \oplus B\right) \cap A^{c}
$$

where $X$ is the 'filled in' portion of $A$. This method, however, while being very simple to implement, requires that the point $p$ be assigned a value of one. This $p$ must be chosen to lie within the boundary to be filled, i.e. the point $p$ must lie within the boundary formed by the outer edges of the icon or text. This requires manual intervention and eliminates the objectivity of the process. A much simpler way would be instead to fill the object from the border in towards the boundary of the icon, instead of from the point $p$ out, and 
then take the complement of this result. A one pixel border with values of one is very simple to implement automatically and eliminates the need to manually select a point $p$. This makes the fill process completely automated. A border is added to the edge detected image as follows:

$$
X(m, n)_{\text {border }}=\left\{\begin{array}{cc}
X(m, n)=1 & \{X \mid X \in \text { Border }\} \\
X(m, n)=0 & \text { elsewhere }
\end{array}\right.
$$

and a Border is defined as the set of all pixels that are contained in the first column, last column, first row, or last row of a digital image. The result of this step is shown in Figure $3-13$.

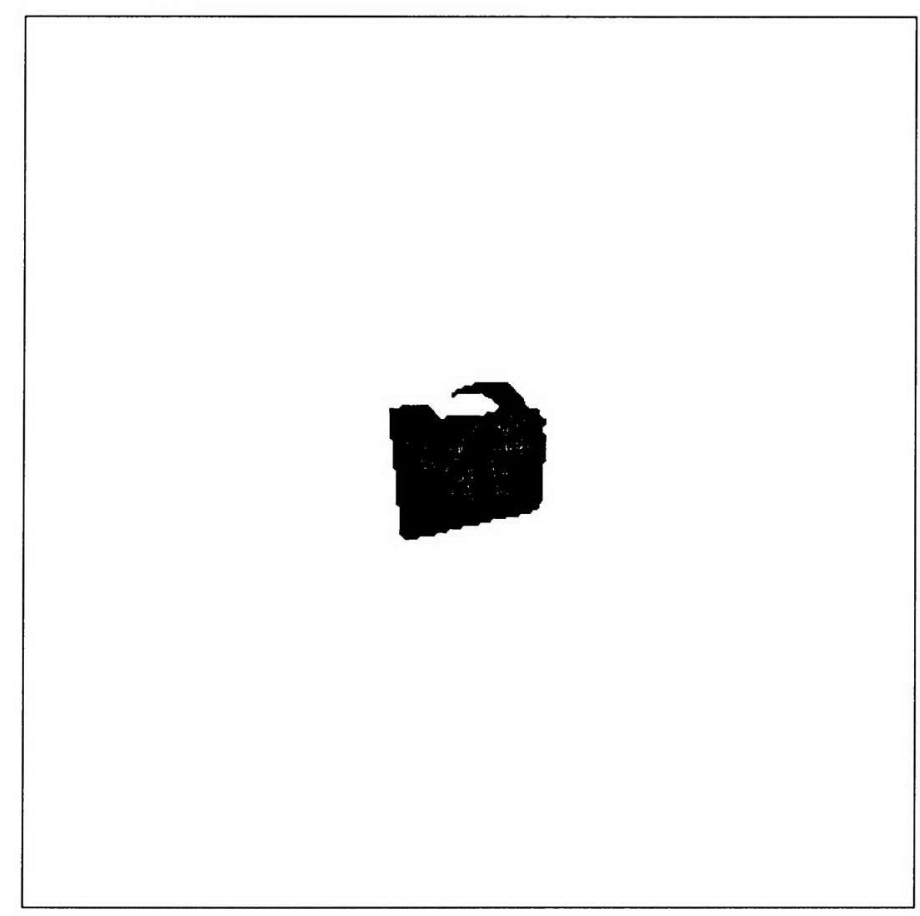

Figure 3-13 Filled in edge detected icon shown in Figure 3-11

Often, the result of the processing the object to extract its spatial extent (from the dilation process) yields a rendition that is slightly larger in area than what the icon truly 
occupies within the object. This is remedied by eroding the filled-in icon to reduce its extents. Thus, the final step is to erode the image by a pyramidal structuring element $[17$, 26]. In words:

"The erosion of A by B, denoted by $\bullet$, is the set of all points $\mathrm{z}$, such that $\mathrm{B}$, translated by $z$, is contained in A." [17]

$$
A \bullet B=\left\{z \mid\left[(B)_{z} \cap A\right] \subseteq A\right\}
$$

A graphical summary of the image segmentation portion of the algorithm is shown in Figure 3-14. The final result of the image segmentation process is shown in Figure $3-14 \mathrm{e}$. Once the object is segmented, this result can be used to create a mask that isolates the relevant regions of the raw precompensated object, separating it from regions in the precompensated object that are not necessary for a clear retinal projection.

In order to create the mask, the segmented area derived from the icon is used in conjunction with the PSF to determine the spatial extent to which information in the precompensated object is needed for reconstruction. This is done by first blurring the segmented image with the PSF. This will yield knowledge of the spatial region containing all image points that would receive a significant impact by blurring any of the points of the object with the PSF under study. The act of convolving the segmented object with the PSF produces an image that differs from the background value of zero only where the PSF would have an impact. An example of this is Figure 3-15, where the segmented image (Figure 3-15a) is shown with an outline delineating the extent to which the PSF affects the icon. The same outline is shown superimposed on the blurred segmented object (Figure 3-15b). 
(a)

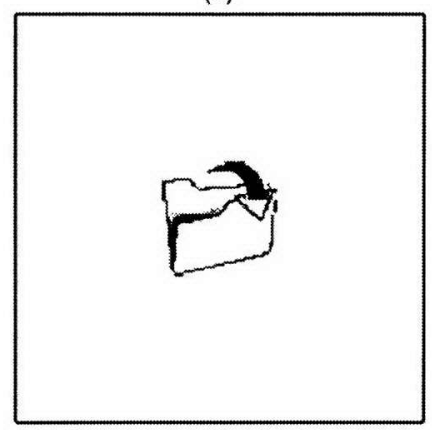

(d)

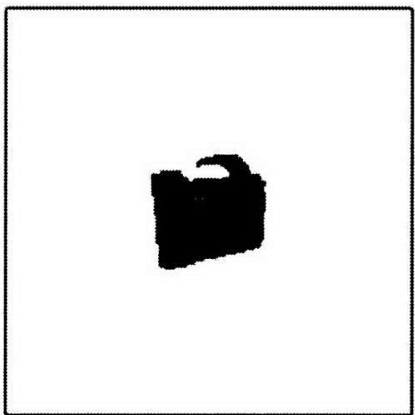

(b)

(c)

(e)

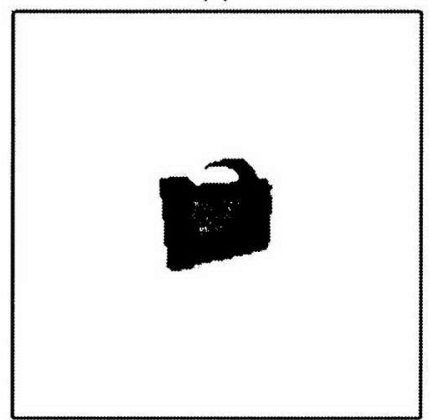

Figure 3-14 (a) Original Image (b) Edge-detected (c) Dilated (d) Filled-in (e) Eroded
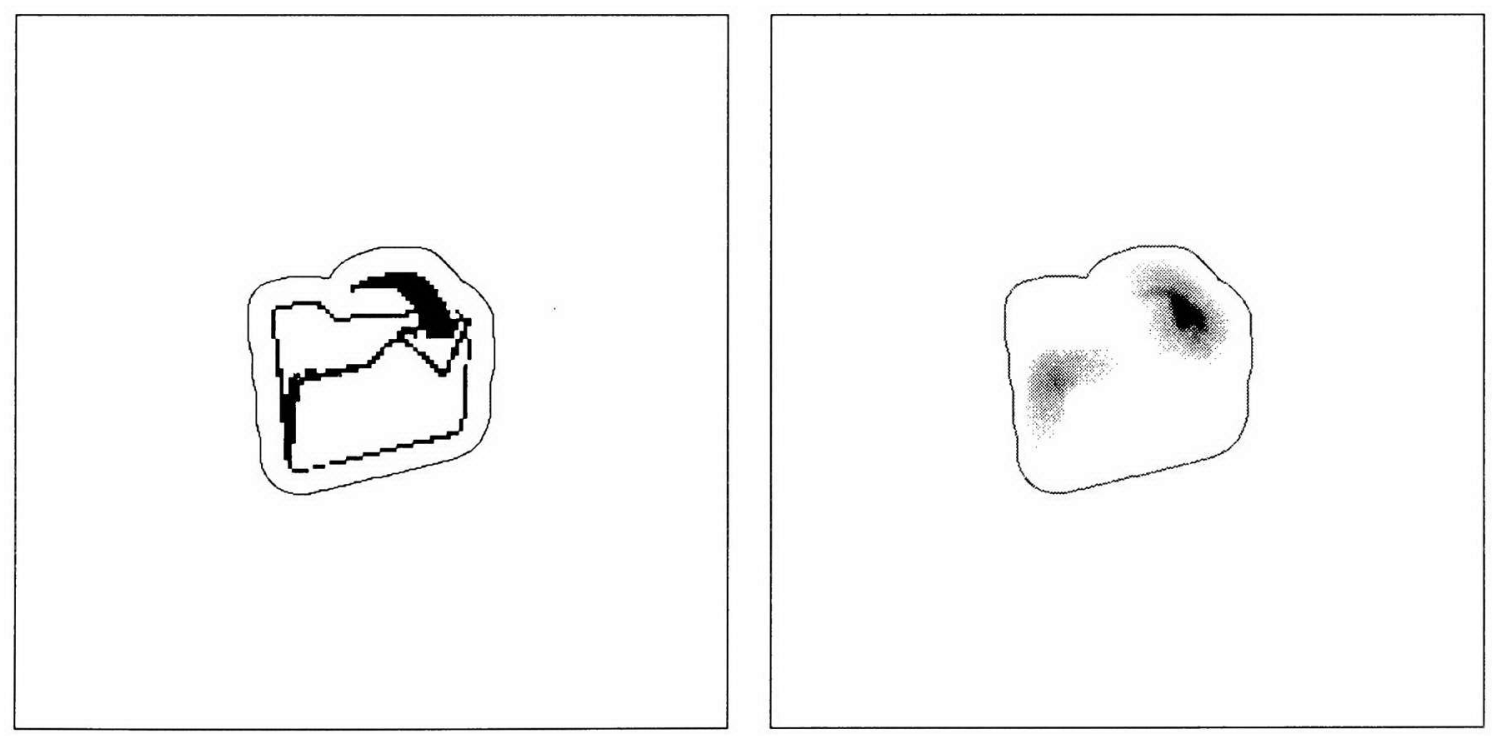

Figure 3-15 Outline superimposed on original (left) and blurred (right) icon 


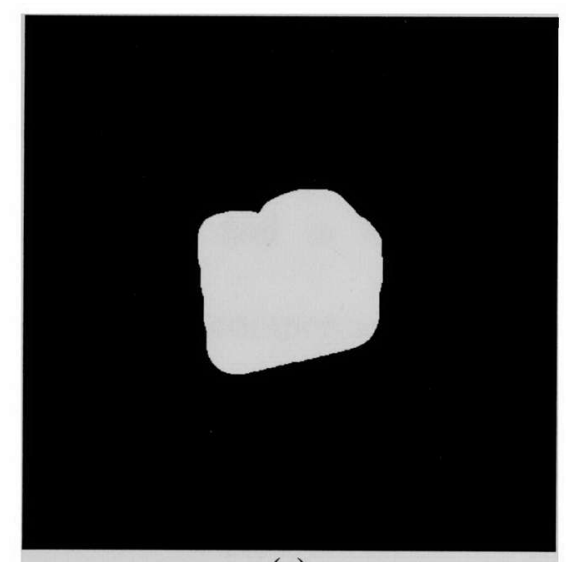

(a)

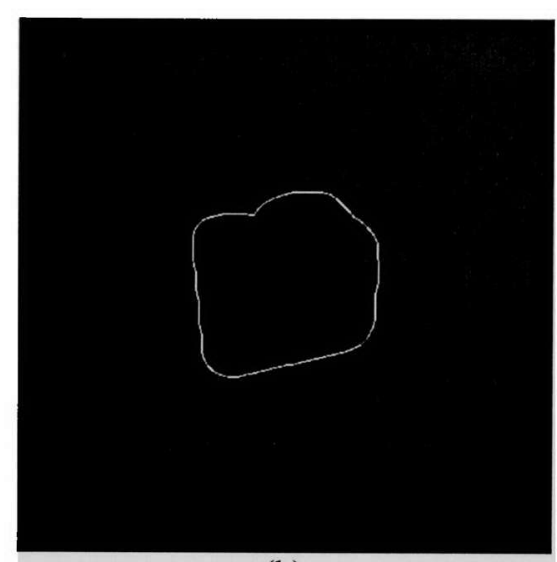

(b)

Figure 3-16 (a) Image Mask (b) Mean Mask

The procedure is summarized as follows:

1. Segment the object to be precompensated using the method described above.

2. Convolve the segmented image (Figure 3-14e) with the user's PSF using Equation 3-2, where $O(x, y)$ is the edge-detected image, $\operatorname{PSF}(x, y)$ is the user's PSF, and $I(x, y)$ is the blurred, segmented image Figure 3-15b.

3. Take the grayscale complement of $I(x, y)$ to produce $I_{c}(x, y)$.

4. Threshold $I_{c}(x, y)$ at a very small value, close to zero, and replace all values above this threshold with one, and values below it with zeros, to produce the mask, $M(x, y)$. (e.g., Figure 3-16a).

5. Multiply the Precompensated object, $O_{p c}(x, y)$, point-to-point with $M(x, y)$.

Although the PSF associated with some aberrations (e.g., Keratoconus) may not be circularly symmetric [1], this method allows for a robust generation of the appropriate mask based on the user's PSF. 


\subsubsection{Integration of Spatial and Spectral Modalities}

Step five above results in a precompensated object with an artificially introduced black background and is not suitable for comfortable identification of the letters displayed. This precompensated object, when convolved with the PSF of the user, will yield a retinal projection of the object with a "halo" around it, as shown in Figure 3-17a. Conversely, arbitrary replacement of a completely white background also gives rise to a "halo" effect that would also be distracting (Figure 3-17b). In order to alleviate this, a background level closer to the unprocessed projected retinal image of the precompensated object is necessary. This can be easily achieved by replacing the background intensity with the mean intensity located at the border of the masked retinal image. By edge-detecting the mask itself using the methods for edge detection described above (Equations 3-14 and 3-15), a sub-mask can be produced that only extracts information from the image at the edge of the mask (Figure 3-16b).

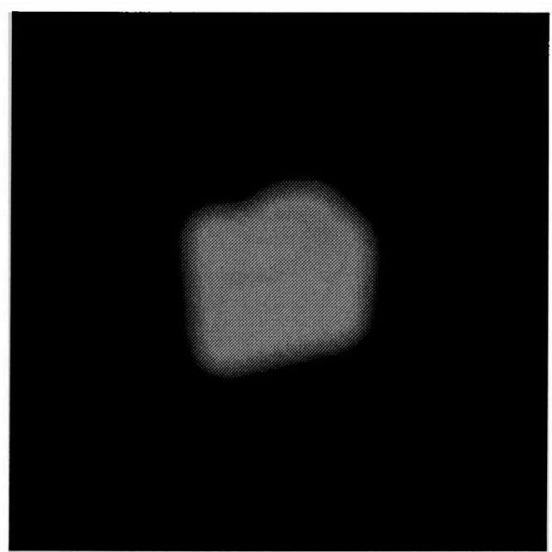

(a)

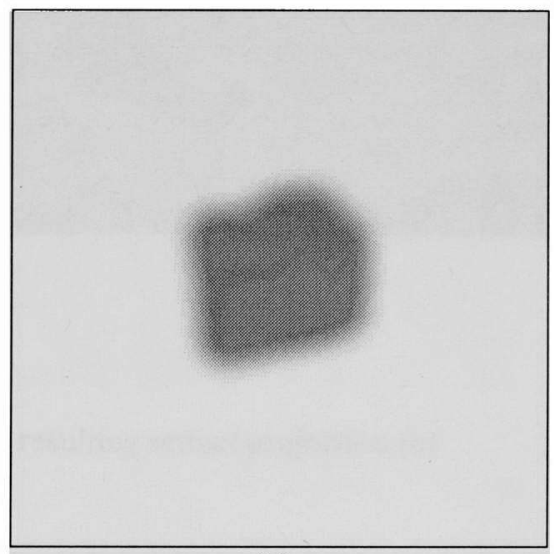

(b)

Figure 3-17 Black (a) and white (b) artificial backgrounds

If the image in Figure $3-16 \mathrm{~b}$ is then multiplied point-to-point with the simulated retinal projection of the shifted and scaled precompensated object and the mean of only 
the non-zero values is calculated, an estimate of the appropriate background level at the edge of the mask is obtained.

The integration of this mean level with the masked precompensated object is implemented using the following equation:

$$
O_{P C m}(x, y)=O_{P C}(x, y) \times M(x, y)+(1-M(x, y)) \cdot m n
$$

where $m n$ is the estimate of the appropriate background intensity, $O_{P C m}(x, y)$ is the masked precompensated object and $\times$ denotes point-to-point multiplication. Figure 3-18 shows the result of this final step, and the corresponding simulated retinal image.

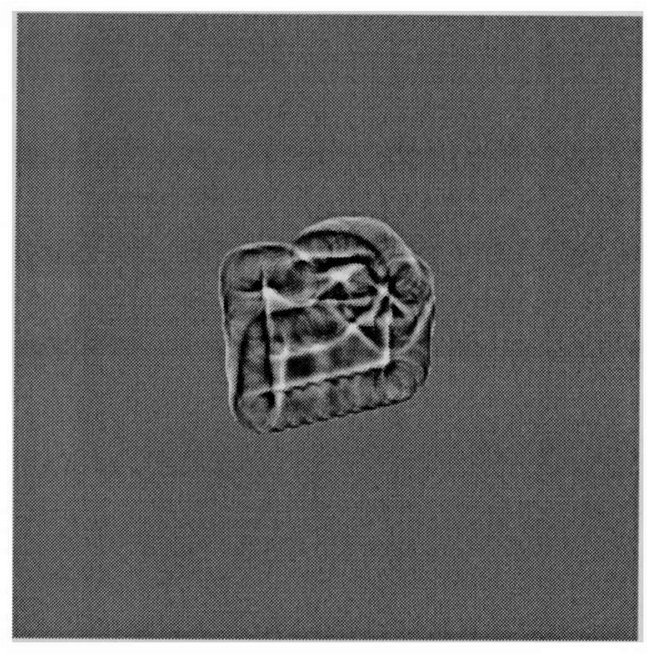

(a)

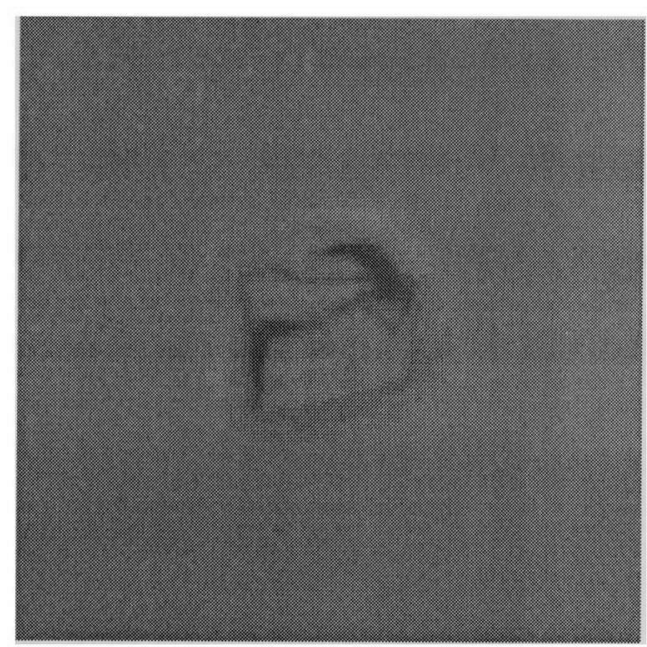

(b)

Figure 3-18 Precompensated image (a) and resulting retinal projection (b)

\subsection{Practical Implementation}

The PSF is not a static characteristic of the human eye. It depends on several factors including pupil diameter, viewing distance, accommodative state, and most importantly the wavelength of light entering the eye. Additionally, the index of refraction 
of any optical medium, which includes the human eye, is a function of the wavelength of light traveling through that medium [48]. Consequently, since the implicit parameters of the PFS are the index of refraction and the wavelength of the incoming light, changes in either parameter will cause the point spread function to vary.

\subsubsection{LCD Considerations}

Humans perceive color through the cones present in the retina. These cones can be divided into three principal sensing categories, corresponding to red, green and blue [17]. Approximately $65 \%$ of all cones are sensitive to red light, $33 \%$ are sensitive to green light, and only $2 \%$ are sensitive to blue light (the blue cones however are the most sensitive). The peak absorption of the red, green, and blue cones occurs at wavelengths of $575 \mathrm{~nm}, 535 \mathrm{~nm}, 445 \mathrm{~nm}$, respectively. The additive nature of light allows for displays to leverage on the fact that the human eye is sensitive in these three spectral regions, allowing users to perceive many colors by only using three wavelengths of visible light.
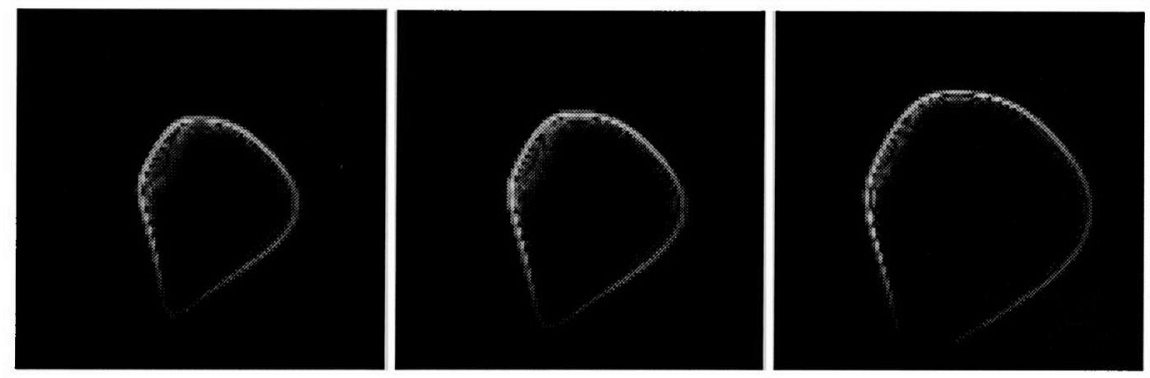

Figure 3-19 From the left, Red PSF, Green PSF, Blue PSF of a user with mild myopia

LCD panels are capable of producing light in the three color ranges, red, green and blue. The RGB convention for representing images specifies that one color pixel be represented as a combination of modulated intensities in each of the three available colors for display, red, green, or blue. This allows most displays to represent millions of colors. 
Thus, in order to simulate the images that a user with a refractive visual aberration would perceive, each channel of the RGB image should ideally need to be processed by its corresponding PSF. Figure 3-19 shows the PSF of a person with mild myopia at the peak absorption levels stated above (all 3 panels share the same scaling).

The objects considered thus far in this dissertation have been monochromatic images, in which each pair of spatial coordinates is associated with a single value: the grayscale level for that "pixel" ranging from a minimum value of 0 to a maximum value of 255. This framework has allowed the verification of the concepts proposed in mathematical simulations, as well as with an "artificial eye" $[1,2]$. However, as mentioned above, it is well known that most commercially available LCD displays are not monochromatic, but rather, are polychromatic based on the RGB color model. Even the black-and-white "binary" text images or icons, when displayed on an LCD panel, contain information in each of the three color components. Therefore, when a "grayscale image" is presented on the LCD panel to a viewer with visual aberrations, the blurring process in his/her eye will comprise three convolution processes. Each channel of the LCD panel, the red, green and blue, will be affected by its appropriate red, green, or blue PSF [31]. Therefore, in order to address the shortcomings of monochromatic precompensation, each color channel must be independently precompensated with the appropriate color PSF and then integrated to form a color precompensated object.

The fact that a polychromatic approach for precompensation is necessary presents a difficulty when trying to generate precompensated objects for display on this LCD monitor, because the three different aberration processes, one for each color channel, 
should be reverted independently. Fortunately, wavefront analyzers provide the PSF for a specific wavelength of light, $840 \mathrm{~nm}$, allowing the calculation of the wavefront aberration that corresponds to that specific wavelength. The wavefront aberration can then be corrected by applying an adjustment factor based on empirical measurements of the chromatic characteristics of the human eye. The amount of adjustment needed for generating the red, green, and blue PSF (RGB PSF) is calculated based on the wavelength at which the wavefront measurement was taken. The process of adjusting the wavefront aberration for a particular wavelength is described in section 3.3.4. Once the adjustment factors are computed, generation of 3 separate red, green, and blue compensated images for display can be created based on the RGB PSF.

Additionally, two problems arise in the precompensation of color images for the LCD panel used in this dissertation. Firstly, even if all three channels are compensated, the typical blue PSF is substantially broader, as shown in Figure 3-19. Compensation for a broad PSF (e.g. blue PSF) proves to be more difficult due to the finite energy capabilities of the LCD display. An LCD panel is not capable of displaying an infinite amount of energy needed for the perfect reconstruction of a compensated image [1].

Secondly, upon characterizing the specific LCD display used for testing with a portable spectrometer described in [24], it was found that the maximum of the spectral profile of each color channel was not near the peak wavelengths of absorption in the human eye. Thus, each color PSF, the red, green, and blue PSF respectively, must be computed for the appropriate wavelengths of light being emitted from the LCD panel. 
Also, the full width at half maximum (FWHM) of the spectral profile for the blue wavelength is very large, as compared to the other two wavelengths. These findings not only require the polychromatic PSF to be "tuned" to the LCD panel, but also present a problem when trying to compensate the blue channel.
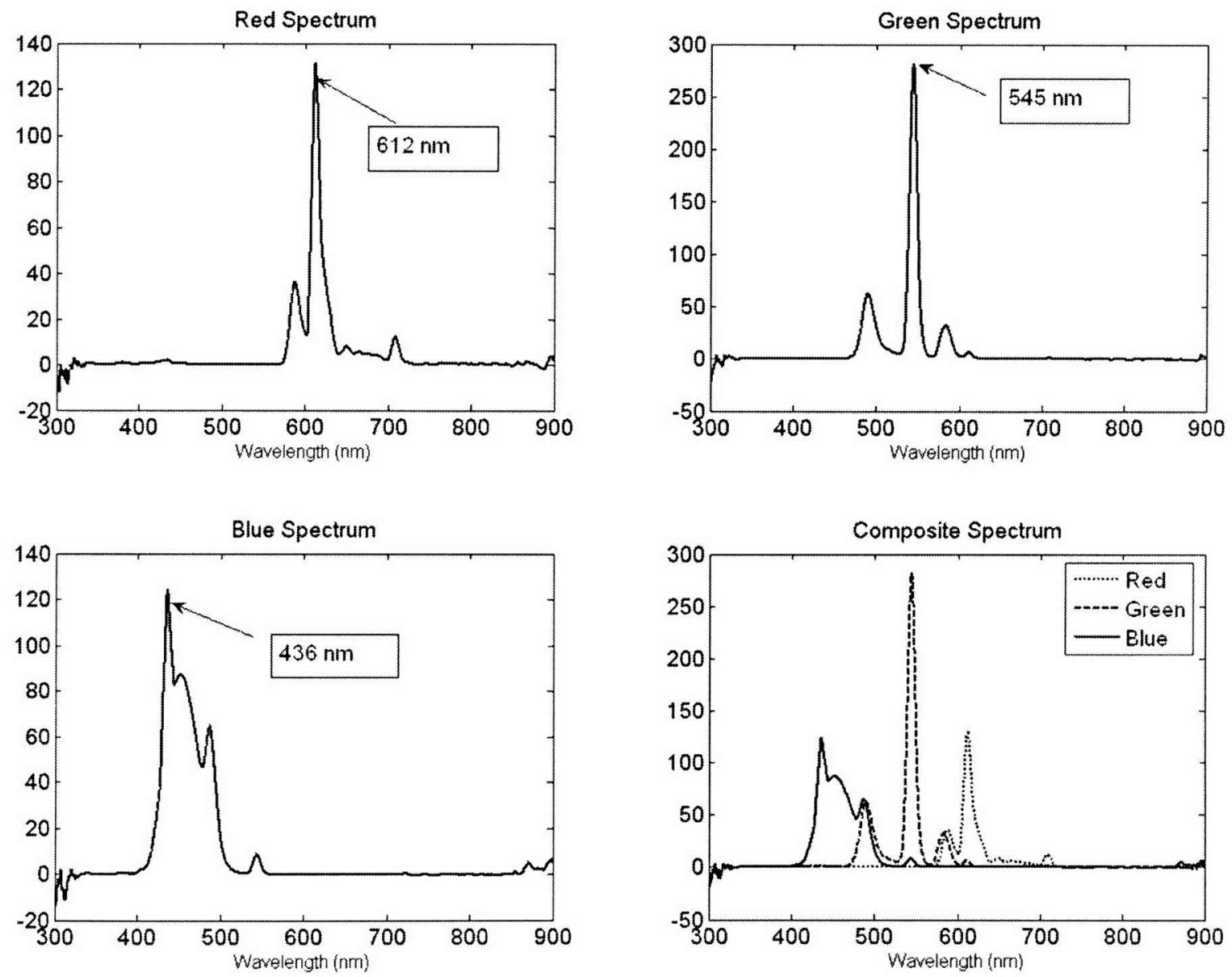

Figure 3-20 Spectral Profile of the Red pixels (top left), Green pixels (top right), Blue pixels (bottom left), Composite (bottom right) as measured on a SONY Model: SDM-S204 LCD display

The spectrum of the blue channel of the LCD panel used is substantially broader than that of the red or green channels (shown in Figure 3-20 bottom left). This results in the blue channel not having a single, easily identifiable PSF, in addition to being the largest of the three color PSFs. Therefore, blue precompensation would not be practical 
because even if multiple blue PSFs were computed, only one can be implemented in the display of the precompensated object.
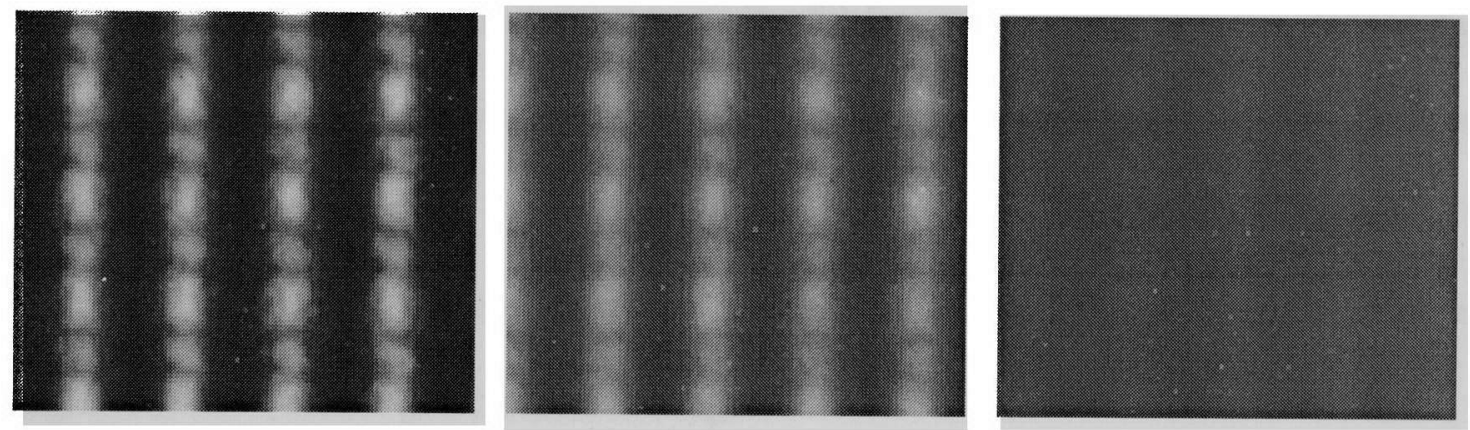

Figure 3-21 Red (left), Green (middle), Blue (right) Channels of the LCD panel captured through a high resolution, machine vision camera. The lens is adjusted for proper focusing of the red channel image.
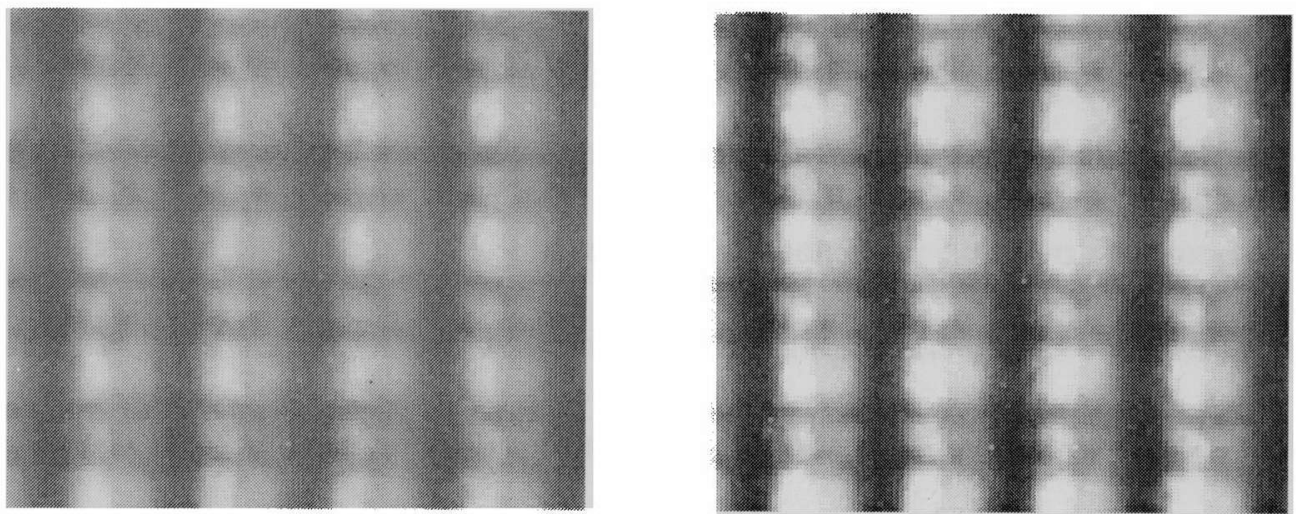

Figure 3-22 RGB (i.e. White Background) (left) vs. RG (i.e. Yellow Background) (right)

Accordingly, it was concluded that the best way to address the wide spectrum of blue being emitted from the LCD panel was to eliminate the blue channel from the RGB object. For the images used in this dissertation, this results in a yellow background. The improvement from using a yellow background versus a white background can be seen in Figure 3-22, where high resolution images of the pixel content of the LCD panel used in testing are shown. 
Figure 3-21 and Figure 3-22 contrast the three color channels displayed in the LCD panel that were used for testing the precompensation algorithm. The red and green channels (Figure 3-21 left and middle, respectively), are very similar in focus. This is due to the fact that the PSF for the red and green wavelengths $(612 \mathrm{~nm}$ and $545 \mathrm{~nm}$, respectively) are very similar in size, as shown in Figure 3-19. The blue PSF (Figure 3-19 far right) is significantly larger resulting in the blue channel image being blurrier as compared to the red and green channel images.

\subsubsection{Pupil Diameter Effects}

The pupil diameter of the human eye is one of the primary factors in computing the PSF, regardless of the wavelengths being considered. Any departure from the pupil diameter at the time of the wavefront aberration measurement will cause the PSF to be different at the time of testing [12]. The wavefront aberration function is composed of the gradient-field components of the wavefront error, sampled at discrete points in the exit pupil of the eye $[12,45]$. At the time of computing the Zernike coefficients that approximate that wavefront, an aperture radius specifying the circular area being sampled must be defined. The pupil size is then the limiting factor in recording because the wavefront function, as measured, is only defined within the natural pupil of the of the eye at the time of measurement [12].

Two consequences arise from using wavefront aberrations that are measured prior to the viewing of precompensated images. Firstly, if the pupil diameter at the time of measurement differs from the pupil diameter at the time of viewing, there will be a difference in the PSF. Two possible scenarios can occur: 
a. The pupil diameter is smaller at the time of measurement than at the time of viewing

b. The pupil diameter is larger at the time of measurement than at the time of viewing

Both scenarios will cause the PSF to differ substantially from the time of measurement to the time of viewing. However, there is a solution for case b. Recently Campbell developed a matrix method to find a new set of Zernike coefficients from an original set in the case when the aperture radius is changed. This new set is formed by multiplying the original set of coefficients (organized as a vector) by a conversion matrix formed from powers of the ratio of the new to the original aperture and elements of a matrix that forms the weighing coefficients of the radial Zernike polynomial functions [12].

Conversely, if the situation is described by case a, the only alternative is to extrapolate the wavefront aberration function out to the larger pupil diameter. This will provide a PSF, but there is no guarantee that the extrapolated wavefront is the actual wavefront of the user, as it is known that the high-order terms diminish as the pupil diameter decreases [23].

Secondly, the pupil diameter, as well as the accommodative state of the eye varies with luminance conditions. Therefore, if the pupil is allowed to change in size, a realtime pupil diameter measurement is required to continuously update the wavefront aberration function (originally obtained at a maximum pupil diameter) for the computation of the PSF. The computation time of the PSF is approximately 30 seconds, 
which implies that even if a real-time pupil diameter is available, the maximum frame rate of the precompensated object 1/30 frames per second (excluding the time necessary for the Fourier transforms and matrix point-to-point multiplication necessary to generate the precompensated object).

In order to minimize the pupil diameter effect due to variations in illumination, the subjects who participated in the testing of this research had their pupils pharmacologically dilated at the time of testing. Two solutions were administered to the subjects by qualified physicians at the time of the patient study. A combination of tropicamide and phenylephrine was used to dilate the pupil, which additionally also reduces the accommodative ability of the eye [10].

\subsubsection{Finite Viewing Distance}

The Zernike polynomials that are generated from standard aberrometers, such as the Wavefront Sciences, COAS-HD unit (http://www.wavefrontsciences.com) typically measure the wavefront aberration of the human eye in its accommodation-free state, i.e. the state of the eye as if it is viewing a point at infinity [15]. This poses a problem when considering images at finite distances because the PSF generated from the wavefront aberration data describes how a user's eye would distort an object if the object was placed at infinity. For the purposes of this dissertation, the object of interest is a computer-generated image displayed on an LCD panel, approximately $50 \mathrm{~cm}$ away from a user's eye. Thus, the wavefront aberration data must be adjusted to account for this finite viewing distance. 
Because the wavefront aberration is measured with respect to a plane wave [15, 31], the adjustment is made by re-referencing the wavefront aberration from a plane wave (which would be generated for a point at infinity [48]) to a wavefront that originates from a point at the desired finite viewing distance [1]. Figure 3-23 illustrates this idea.

(a)

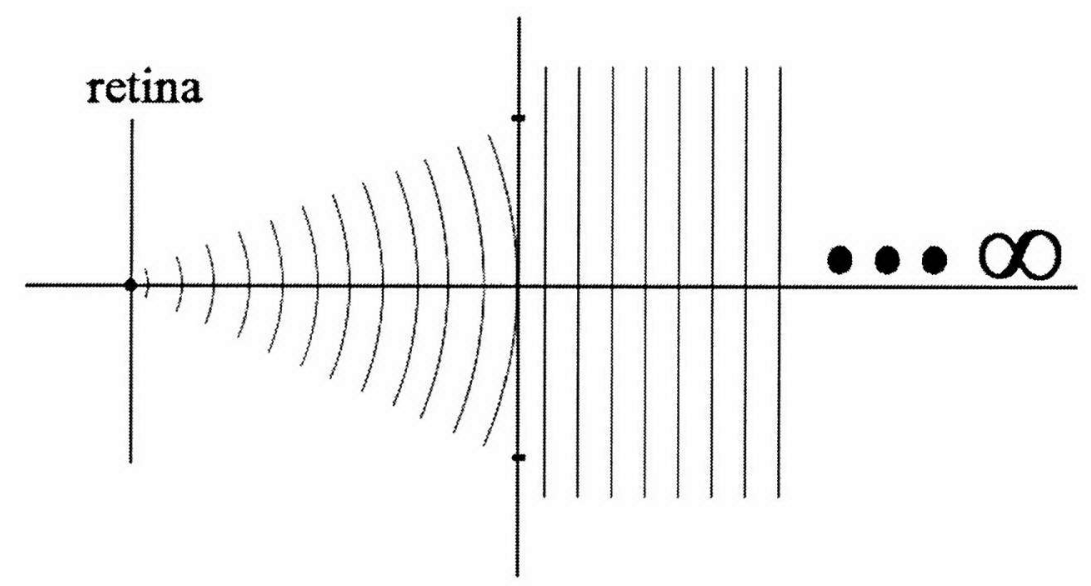

(b)

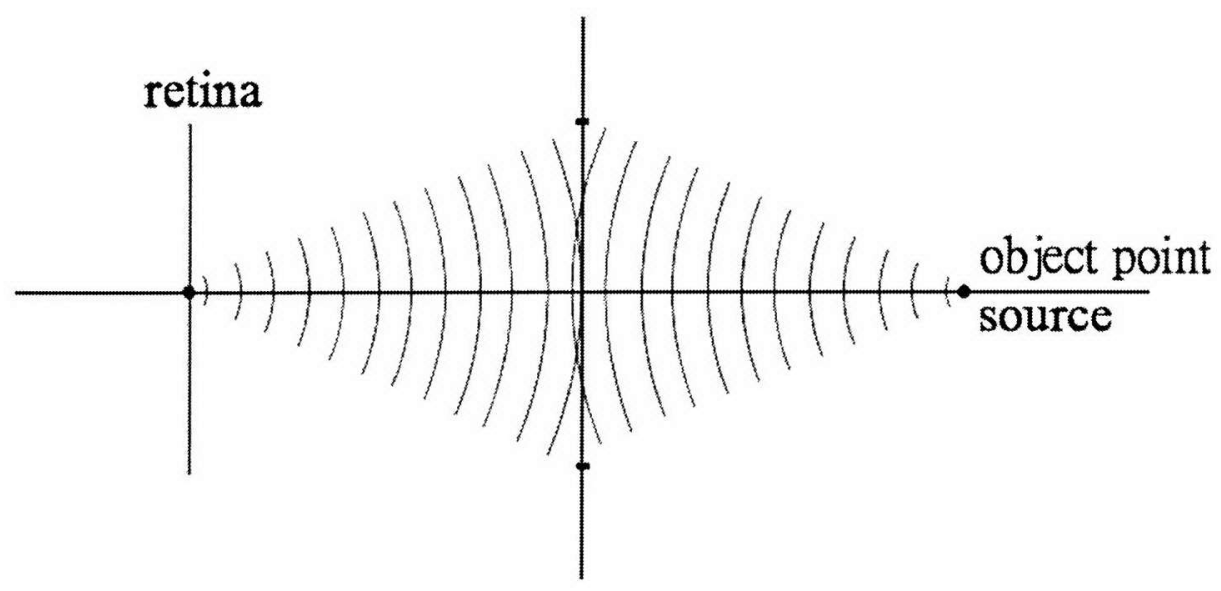

Figure 3-23 Illustration of how plane waves (infinite distance point source) (a) and spherical waves (finite distance point source) are focused on the retina 
Thus, the wavefront from a finite distance object should be added to the measured wavefront. One can view this operation as adding a wavefront aberration to the eye consisting of a positive wavefront (as defined by the positive axis taken to be from the pupil out towards the object point source).

In order to determine the wavefront caused by a point located at a finite distance away from the eye, it is common practice to approximate this wavefront from the sag of a circle with a radius equal to the distance between the object and the eye, at a radial distance PR from the apex [35]. This is shown in Figure 3-24. This wavefront aberration due to the finite viewing distance is computed at the pupil extent.

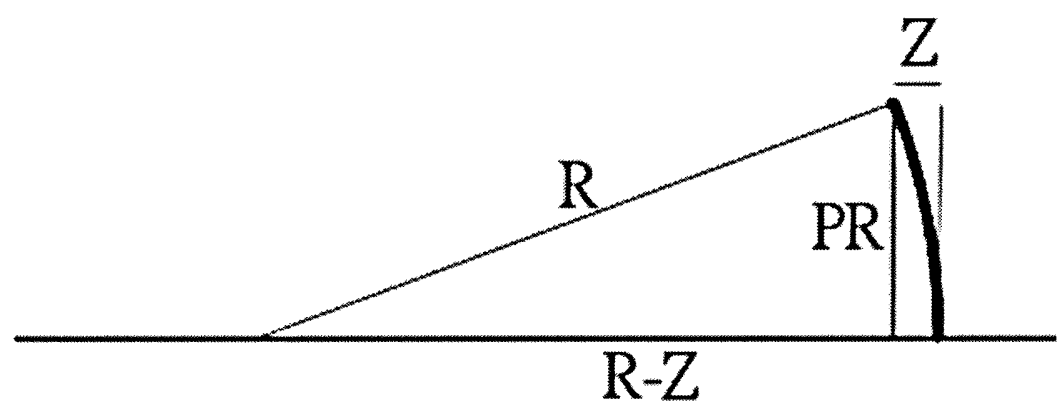

Figure 3-24 Geometry used to find wavefront error $(Z)$ due to viewing a point at a distance $(R)$ away from the eye

The derivation is as follows:

$$
\begin{array}{ccc}
P R^{2}+\left(R-Z_{o b j}\right)^{2} & = & R^{2} \\
\left(R-Z_{o b j}\right)^{2} & = & R^{2}-P R^{2} \\
R-Z_{o b j} & = & \sqrt{R^{2}-P R^{2}} \\
Z_{o b j} & = & R-\sqrt{R^{2}-P R^{2}}
\end{array}
$$

where $Z_{o b j}$ is the wavefront emerging from an object located at a finite distance in front of the eye, $R$ is that distance, and $P R$ is the pupil radius. 
A Taylor series expansion can be applied to Equation 3-21 to approximate the square root to the first order. The Taylor series expansion of $\sqrt{1+x}$ is

$$
\begin{aligned}
& \sqrt{1+x}=\sum_{n=0}^{\infty} \frac{(-1)^{n}(2 n) !}{(1-2 n) n !^{2} 4^{n}} x^{n} \\
& \text { for }|x|<1
\end{aligned}
$$

The result of applying the Taylor series expansion yields

$$
Z_{o b j}=\frac{P R^{2}}{2 R}
$$

where $Z_{o b j}$ is the Zernike coefficient due to the object, $P R$ is the pupil radius, and $R$ is the object distance. According to Equation 3-21, this will only be valid as long as $P R^{2}<R^{2}$, which will always be true for the practical viewing distance (used in this dissertation) of $\mathrm{R}=0.5 \mathrm{~m}$. This distance will always be larger than the maximum pupil diameter of the human eye.

Instead of computing the entire wavefront aberration surface and then adding it to the non-planar surface generated by the finite distance object, an equivalent operation can be performed on the second-order coefficient in the Zernike polynomial. The secondorder term of the Zernike polynomial corresponds to a sphere, which is the same surface that is generated by the finite object point source. This can be done because the Zernike polynomials are orthogonal, and thus can be manipulated independently of each other [49]. 


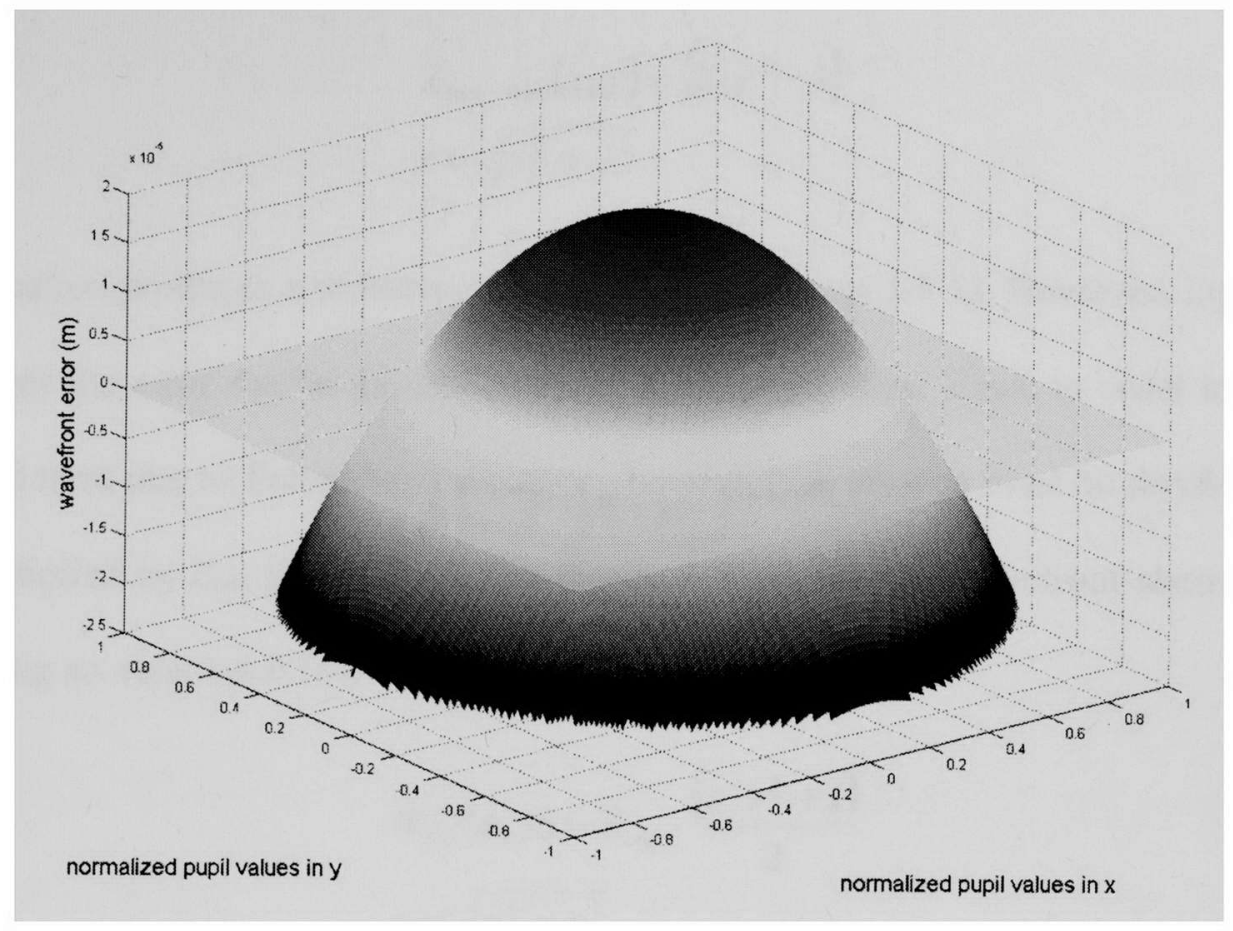

Figure 3-25 2-D wavefront aberration function for $Z_{\mathrm{def}}=-2 \times 10^{-3} \mathrm{~m}$ with a semi-transparent zero plane The Zernike polynomial for second-order sphere is

$$
\begin{aligned}
& W_{s p h}(x, y)=Z_{s p h} \cdot\left(2 \cdot r^{2}-1\right) \\
& r=\sqrt{x^{2}+y^{2}}
\end{aligned}
$$

where $W_{s p h}$ is the wavefront function generated across the pupil plane, $Z_{s p h}$ is the Zernike coefficient corresponding to $2^{\text {nd }}$ order sphere, and $x$ and $y$ are normalized units across the pupil plane $[35,49]$. Figure $3-25$ shows the two-dimensional wavefront aberration function corresponding to a Zernike defocus term equal to $-2 \times 10^{-3} \mathrm{~m}$, which is equivalent to -4 Diopters of defocus for a pupil radius of $4.5 \mathrm{~mm}$.

The nature of the Zernike polynomials makes the term for defocus be bipolar, with the surface for defocus defined as 


$$
\begin{aligned}
& Z_{\text {sph_surf }}(x, y)=\left(2 \cdot r^{2}-1\right) \\
& r=\sqrt{x^{2}+y^{2}}
\end{aligned}
$$

This equation produces a prototype surface with a range of [-1 1]. However, Equation 325 defines the error due to the finite object at the pupil edge. Thus, in order to create a spherical term due to finite object distance, the prototype surface must be divided by two and multiplied by $Z_{o b j}$ (Equation 3-26). Figure 3-26 shows the wavefront aberration due to viewing an object at $0.5 \mathrm{~m}$.

$$
\begin{aligned}
& W_{O D}(x, y)=Z_{o b j} \cdot \frac{\left(2 \cdot r^{2}+1\right)}{2} \\
& r=\sqrt{x^{2}+y^{2}}
\end{aligned}
$$

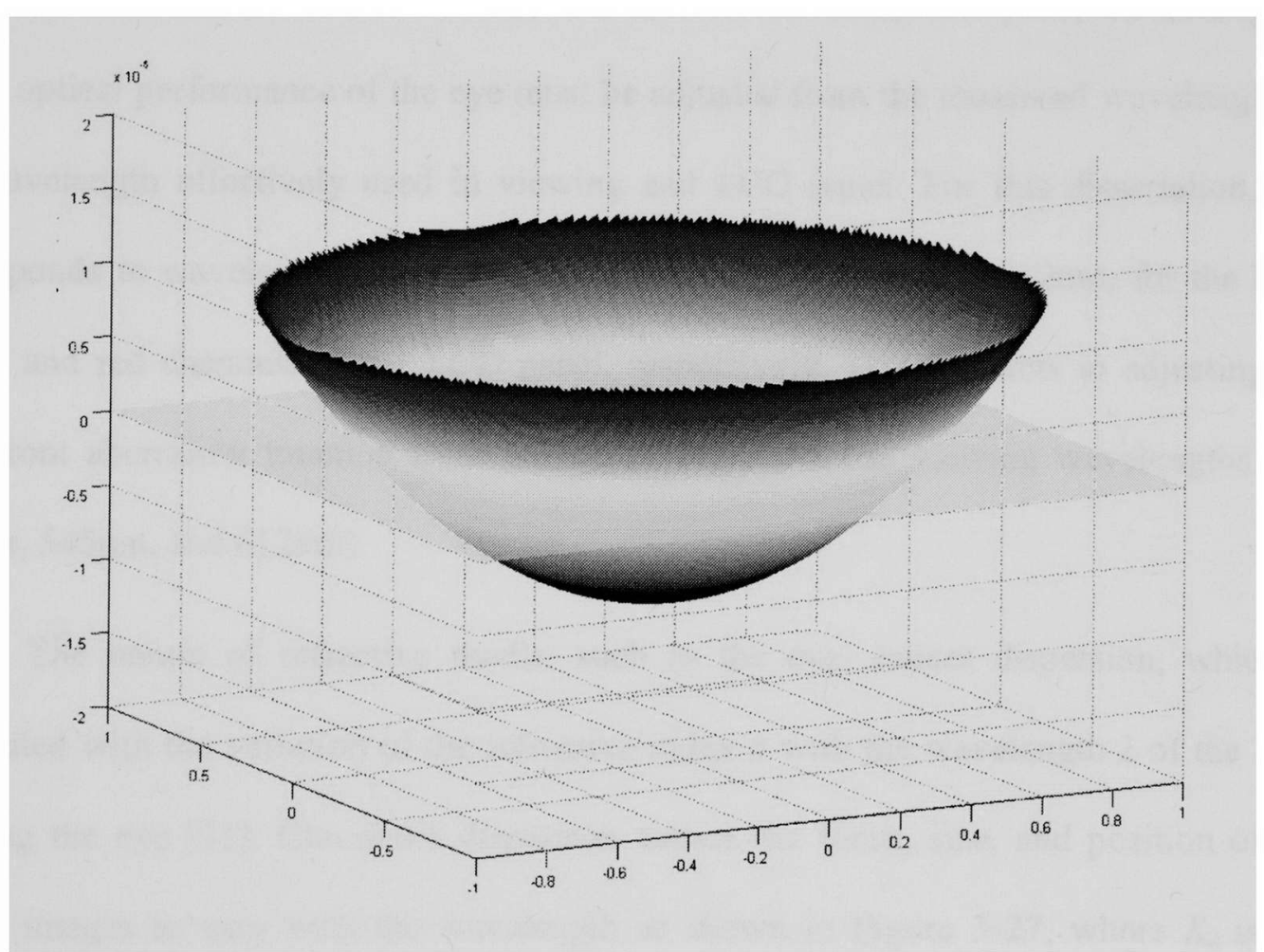

Figure 3-26 2-D wavefront aberration function due to viewing an object at $0.5 \mathrm{~m}$ with a semi-transparent plane zero plane 
In order to produce a new term that accounts for the finite viewing effect, both terms are added together to produce a new adjusted term $W_{s p h_{-} a d j}$.

$$
W_{\text {sph_adj }}(x, y)=W_{s p h}(x, y)+W_{O D}(x, y)
$$

\subsubsection{Chromatic Eye}

As mentioned in section 3.3.1, the LCD display is not monochromatic, but polychromatic. The question arises then, how is the color PSF formed? Three separate convolutions must take place, one for each channel of the LCD, with three distinct PSFs. The wavefront measurements generate Zernike polynomials that describe the wavefront aberration measured at a specific wavelength of light $(840 \mathrm{~nm}$ for the COAS-HD aberrometer from Wavefront Sciences, Inc. used in this research). Thus, the description of the optical performance of the eye must be adjusted from the measured wavelength, to the wavelength effectively used in viewing and LCD panel. For this dissertation, this corresponds to wavelengths of approximately $435 \mathrm{~nm}, 545 \mathrm{~nm}$, and $612 \mathrm{~nm}$, for the blue, green, and red channels of the LCD panel, respectively. This amounts to adjusting the wavefront aberration function from $840 \mathrm{~nm}$ to each of those viewing wavelengths (i.e. $435 \mathrm{~nm}, 545 \mathrm{~nm}$, and $612 \mathrm{~nm})$.

The nature of refractive media, such as the eye, causes dispersion, which is associated with the variation of the refractive index $n$ with the wavelength $\lambda$ of the light entering the eye [31]. Chromatic dispersion causes the focus, size, and position of the retinal images to vary with the wavelength as shown in Figure $3-27$, where $K_{\lambda}$ is the change in focus, and the rays of short wavelengths (S) are represented with broken lines, and the rays of long wavelengths $(\mathrm{L})$ are represented with solid lines. 
a $\Delta$ Focus $=K_{\lambda}$

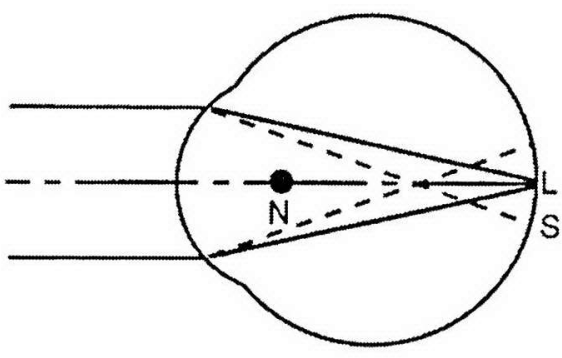

b

$\Delta$ Magnification $=z \cdot K_{\lambda}$

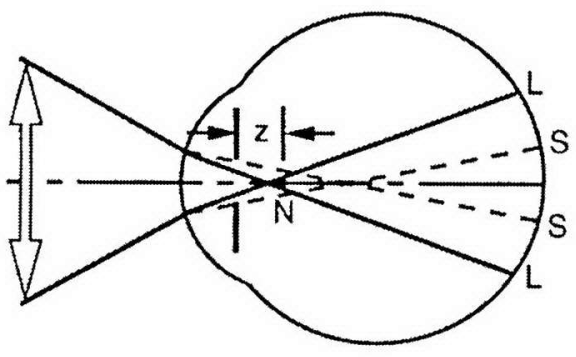

C

$\Delta$ Position $=\tau=K_{\lambda} z \sin \omega$

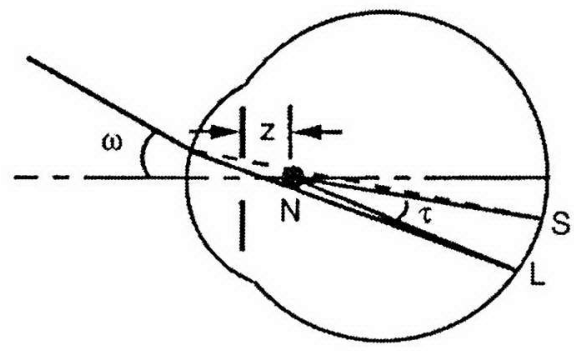

Figure 3-27 Chromatic aberrations cause difference of focus (a), magnification (b), and position (c) [31]

The magnification effect is negligible for the natural eye, being about $1 \%$ [31]. The change in position can also be neglected, given the fact that objects being precompensated will fall in line with the optical axis of the eye and they will be relatively small compared to the field of view of the human eye (a maximum of $14^{\circ}$ of visual angle for the icons vs. $180^{\circ}$ for the complete field of view of the human eye). 
Thus, the only factor that must be accounted for is the change in focus due to chromatic dispersion. Fortunately, Thibos et al. have developed an eye model called the "Indiana Eye", which can simultaneously account for chromatic aberrations, as well as the wavefront aberrations of the human eye [31]. According to this model, the change in focus for a reference wavelength is given by

$$
\Delta \text { Focus }=\frac{n\left(\lambda_{1}\right)-n\left(\lambda_{2}\right)}{n\left(\lambda_{1}\right)}
$$

where

$$
\begin{aligned}
& n(\lambda)=a+\frac{b}{\lambda-c} \\
& a=1.320535 \\
& b=0.004685 \\
& c=0.214012
\end{aligned}
$$

and $\lambda_{1}$ and $\lambda_{2}$ are the reference and desired wavelengths, respectively.

Figure 3-28 shows a plot of change in focus for the visible spectrum at a reference wavelength of $840 \mathrm{~nm}$. Table 3-1 shows the correction factors in units of Diopters (for a typical human eye) used for each of the wavelengths of the LCD panel.

Table 3-1 LCD wavelengths and their corresponding correction factors

\begin{tabular}{ccc} 
Wavelength (nm) & & Correction Factor (D) \\
\cline { 1 - 1 } 435 & & -1.838 \\
545 & & -0.9007 \\
612 & & -0.5785 \\
\hline
\end{tabular}




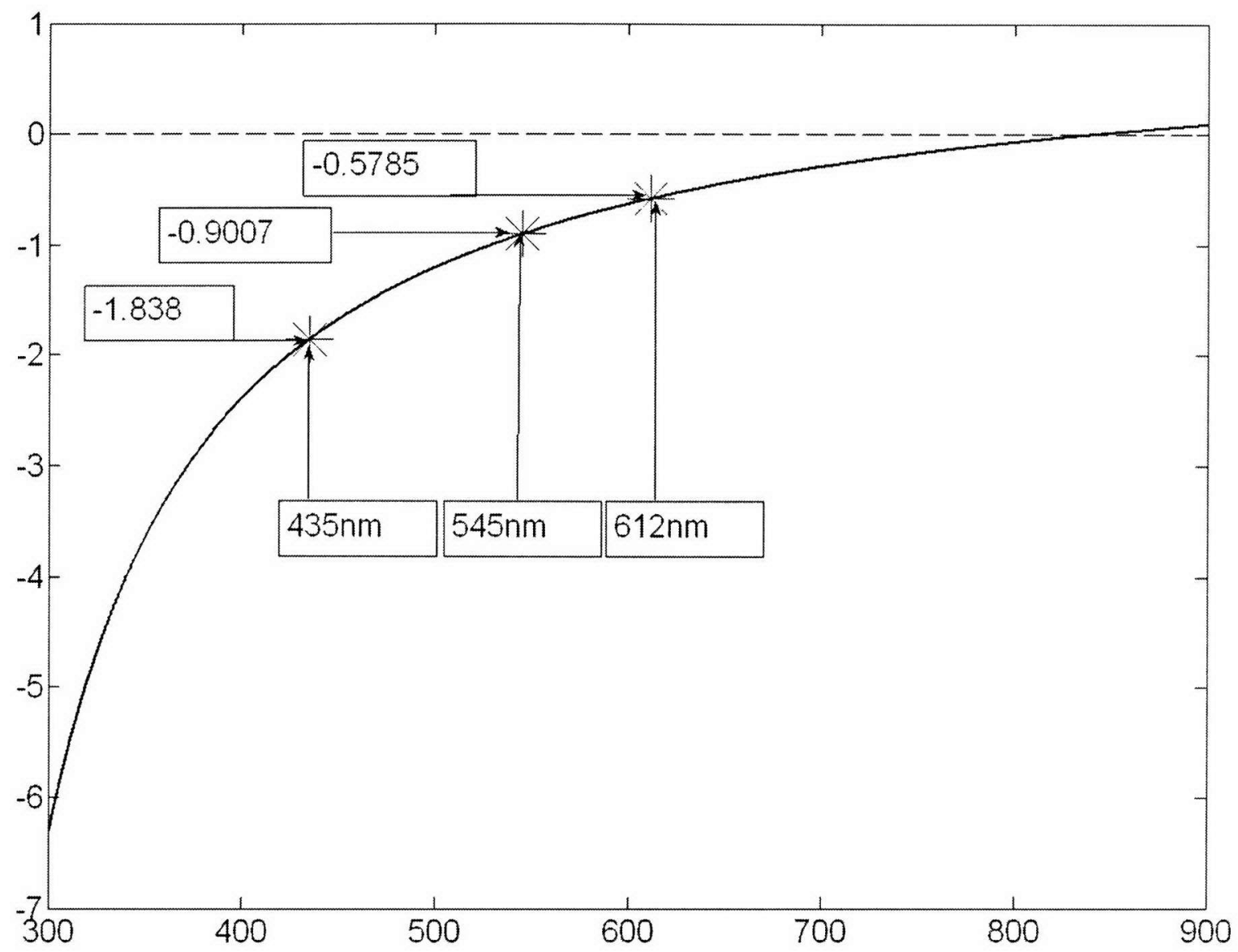

Figure 3-28 Wavelength Adjustment Curve

In order to apply this correction factor to a wavefront aberration function, a similar method to the one employed in section 3.3.3 can be used. The spherical term (in Diopters) for a given second order defocus term is given by

$$
M=\frac{2 \cdot Z_{s p h}}{P R^{2}+Z_{s p h}^{2}}
$$

where $M$ is the amount of defocus of the wavefront aberration (in Diopters), $P R$ is the pupil radius, and $Z_{s p h}$ is the Zernike term due to the wavefront aberration error at the edge of the pupil [35]. 
In order to compute a Zernike adjustment for the three different wavelengths in Table 3-1, Equation 3-30 can be used. Equation 3-30 can be rewritten as

$$
M \cdot Z_{s p h}^{2}-2 \cdot Z_{s p h}+P R^{2}=0
$$

and the quadratic formula

$$
\frac{-b \pm \sqrt{b^{2}-4 \cdot a \cdot c}}{2 a}
$$

can be applied to Equation 3-31 to solve for $Z_{s p h}$, which is given by

$$
Z_{s p h}=\frac{1 \pm \sqrt{1-M^{2} \cdot P R^{2}}}{M}
$$

Using the first order Taylor series expansion for $\sqrt{1+x}$, Equation 3-33 can be written

$$
Z_{s p h}=\left\{\begin{array}{c}
\frac{4-M^{2} \cdot P R^{2}}{2 \cdot M} \\
M \cdot \frac{P R^{2}}{2}
\end{array} .\right.
$$

Initially, the problem arises as to which solution of $Z_{s p h}$ to choose. For a practical viewing distance of 0.5 and a pupil radius of $4.5 \mathrm{~mm}, Z_{s p h}=-3.4572,-4.628 \mathrm{e}-006$, for each equation respectively. The wavefront aberration function is measured on the order of $\mu m$. The first solution will always be on the order of $\mathrm{m}$, excluding it altogether. Thus, the appropriate term to use is

$$
Z_{\text {color }}=M_{\text {color }} \cdot \frac{P R^{2}}{2},
$$


where $Z_{\text {color }}$ is the Zernike adjustment coefficient that is added to the second order Zernike coefficient of the measured wavefront, $M_{\text {color }}$ is the change in focus (in Diopters) for a particular wavelength, and $P R$ is the pupil radius. Therefore,

$$
\begin{aligned}
& W_{\text {color }}(x, y)=Z_{\text {color }} \cdot \frac{\left(2 \cdot r^{2}+1\right)}{2} \\
& r=\sqrt{x^{2}+y^{2}}
\end{aligned}
$$

gives the wavefront aberration adjustment for color, $W_{\text {color }}$, according to $Z_{\text {color }}$. Thus, Equation 3-36 can be used to adjust the measured wavefront for any arbitrary amount of defocus due to various wavelengths.

\subsection{Design of Experiments}

\subsubsection{Software Simulation Experimental Design}

As a first step in validating the effectiveness of the precompensation approach proposed, a software simulation of the precompensation process, including the software simulation of the optical processing of the eye was carried out. Two experiments were designed to asses the improvement that the precompensation processing delivers in retinal image quality to software-simulated optical systems described by unique PSFs using as target objects icons of various sizes. These experiments were performed using MATLAB $^{\circledR}$.

\section{Experiment 1}

Thirty-six spherical aberrations were artificially generated using Equations 3-24 and 3-25. The amounts of defocus were chosen at random in the range of -3 Diopters of defocus to -7 Diopters of defocus. The PSF corresponding to each aberration was 
generated using a viewing distance of $50 \mathrm{~cm}$. Six icons, each at three different sizes, were used as objects in the model of the human optical system given in Equation 1-1 and the output was computed (i.e. the retinal projection of the object). Next, each icon was precompensated using the methods described above, one for each unique PSF. For each of these treatments, the output of the model in Equation 1-1 was computed (i.e., the retinal projection of the precompensated object).

Thus, the experiment can be considered a factorial experiment with three fixed factors: Icon (I) - six levels, Size (S) - three levels, Method (M) - two levels. Each unique PSF was assigned at random to each treatment combination. The dependent variable, based on the output of each trial, is a measure of similarity between the object, and its corresponding retinal projection (described in the following section) for the various treatments. The model for the analysis of variance is as follows:

$$
Y_{i j k m}=\mu+I_{i}+S_{j}+M_{k}+S M_{j k}+\varepsilon_{i j k}
$$

blocking on Icons. Blocking was applied to the Icon variable in order to eliminate the variance that the Icon factor introduces into the model. It is assumed that the Icon factor does not interact with any of the other factors. The variables are shown in Table 3-2.

Table 3-2 Factors for software experiment 1

\begin{tabular}{ccc} 
Fixed Factors & Variable & Range \\
\hline Icon & $I_{i}$ & $i=1: 6$ \\
Size & $S_{j}$ & $j=1: 3$ \\
Method & $M_{k}$ & $k=1: 2$ \\
\hline
\end{tabular}




\section{Experiment 2}

Twenty spherical aberrations were artificially generated using Equations 3-24 and 3-25. The amounts of defocus were chosen at random in the range of -3 Diopters of defocus to -7 Diopters of defocus. The PSF corresponding to each aberration was generated using a viewing distance of $50 \mathrm{~cm}$. Six icons, each at three different sizes, were used as objects in the model of the human optical system given in Equation 1-1 and the output was computed (i.e. the retinal projection of the object). Next, each icon was precompensated using the methods described above. For each of these precompensated icons, the output of the model in Equation 1-1 was computed (i.e. the retinal projection of the precompensated object). This was repeated for each unique PSF.

Thus, the experiment can be considered a repeated measures experiment with three fixed factors: Icon (I) - six levels, Size (S) - three levels, Method (M) - two levels. Every treatment combination was applied to 20 unique PSFs. The dependent variable, based on the output of each trial, is a measure of similarity between the object, and its corresponding retinal projection (explained in the following section) for the various treatments. The experiment is treated as randomized complete block design (RCBD) experiment, taking the subjects as a random variable and blocking the subject variable. The model for the analysis of variance is as follows:

$$
Y_{i j k m}=\mu+I_{i}+S_{j}+I S_{i j}+M_{k}+I M_{i k}+S M_{j k}+I S M_{i j k}+P_{m}+\varepsilon_{i j k m}
$$

where $P$ is the subject variable. The variables are shown in Table 3-3. 
Table 3-3 Factors for software experiment 2

\begin{tabular}{ccc} 
Fixed Factors & Variable & Range \\
\hline Icon & $I_{i}$ & $i=1: 6$ \\
Size & $S_{j}$ & $j=1: 3$ \\
Method & $M_{k}$ & $k=1: 2$ \\
$\begin{array}{ccc}\text { Random Factors } \\
\text { Subject }\end{array}$ & $P_{m}$ & $m=1: 20$ \\
\hline
\end{tabular}

Difference Measure

The Difference Measure is computed as a sum over all pixels of the modulation of the square difference between the object and the retinal image by the Visible Differences Predictor (VDP).

$$
\begin{aligned}
& \left.S M=\sum_{x} \sum_{y} \cdot \operatorname{abs}(\operatorname{Vdg}(\operatorname{Object}(x, y))-\operatorname{Edge}(\operatorname{retprect}(x, y), \operatorname{retproj}(x, y)))\right)^{2} \\
&
\end{aligned}
$$

The VDP provides a map, on a per pixel basis, of the probability that a human observer will detect a difference in a pixel in a reference image as compared to the same reference image after some type of processing has been applied. The reader is referred to [47] for a detailed explanation of VDP. Additionally, $S M$ reflects the degree to which the images are similar, based on the probability of a human observer detecting a difference in the image.

\subsubsection{Artificial Eye Experimental Design}

The next logical step was to test this process in fixed optical system. An artificial eye was designed to simulate the optical system of the human eye. The details of the artificial eye design, including lens designs, are described in Appendix B. This experiment was designed to asses the improvement that the precompensation processing 
delivers in retinal image quality to real optical systems described by unique PSFs using as target objects icons from of various sizes. The experimental protocol was designed using MATLAB $^{\circledR}$, s GUI tools.

Five lenses with arbitrary aberrations were generated using a coating of Norland63 UV cured optical adhesive to provide high-order aberrations. The preparation of these lenses is detailed in Appendix B. After the creation of the five lenses, each lens had a unique aberration that must be measured in a wavefront analyzer to obtain the Zernike polynomials describing the aberration, and subsequently the PSF for each lens. Each PSF corresponding to each aberration was adjusted for color viewing (the lens are adjusted in a similar fashion as the human eye, the details of which are given in Appendix B) and for distance at a viewing distance of $50 \mathrm{~cm}$. Six icons, each at three different sizes, were used as objects to the artificial eye and the output was measured as a digital capture through the CCD of in the artificial eye (i.e. the "retinal" projection of the object). Next, each icon was precompensated using the methods described above and presented as objects to the artificial eye. For each of these precompensated icons, the output was measured as a digital capture through the $\mathrm{CCD}$ of in the artificial eye (.i.e. the retinal projection of the precompensated object). This was repeated for each of the five lenses.

Thus, the experiment can be considered a repeated measures experiment with three fixed factors: Icon (I) - six levels, Size (S) - three levels, Method (M) - two levels. Every treatment combination was applied to the 5 unique lenses. The dependent variable, based on the output of each trial, is a measure of similarity between the object, and its corresponding retinal capture (CCD image) for the various treatments, as described in 
Equation 3-39. The experiment is treated as randomized complete block design (RCBD) experiment, taking the lens factor as a random variable and blocking on it. The model for the analysis of variance is as follows:

$$
Y_{i j k m}=\mu+I_{i}+S_{j}+I S_{i j}+M_{k}+I M_{i k}+S M_{j k}+I S M_{i j k}+L_{m}+\varepsilon_{i j k m}
$$

where $L$ is the lens variable. The variables are shown in Table 3-4.

Table 3-4 Factors for artificial eye experiment

\begin{tabular}{ccc} 
Fixed Factors & Variable & Range \\
\hline Icon & $I_{i}$ & $i=1: 6$ \\
Size & $S_{j}$ & $j=1: 3$ \\
Method & $M_{k}$ & $k=1: 2$ \\
Random Factor & & \\
\cline { 1 - 1 } Lens & $L_{m}$ & $m=1: 5$ \\
\hline
\end{tabular}

\subsubsection{Human Subject Experimental Design}

In addition to assessing how well the precompensation process performs in simulation and for an artificial eye, it is necessary to test how well the precompensation process improves the recognition abilities of human subjects. This was accomplished using two experiments: one to measure the improvement in visual acuity as measured by a standard eye test, and the second to measure the improvement in object recognition.

Twenty subjects were chosen, and out of those subjects, five were controls, i.e. they did not have significant visual refractive errors. The remaining fifteen subjects were chosen as follows: Five subjects were chosen having only myopia, with at least -3 
Diopters of sphere, five subjects were chosen having both myopia and astigmatism, with at least -3 Diopters of sphere and having any amount of astigmatism stronger than -0.5 Diopters, and five subjects were chosen to have been diagnosed with Keratoconus in at least one eye. Table 3-5 shows the subject number and their prescription, along with the group.

Table 3-5 Subject number, group, and prescription

\begin{tabular}{|c|c|c|c|c|c|c|c|}
\hline \multirow[b]{3}{*}{ Subject } & \multicolumn{6}{|c|}{ Prescription } & \multirow{3}{*}{ Group } \\
\hline & & OD & & & OS & & \\
\hline & SPH & CYL & AXIS & $\mathrm{SPH}$ & CYL & AXIS & \\
\hline 1 & -6 & -0.5 & 150 & -6.25 & -0.75 & 180 & 3 \\
\hline 2 & -0.5 & & & -0.5 & -0.25 & 150 & 1 \\
\hline 3 & -5.5 & & & -5.25 & & & 2 \\
\hline 4 & -7 & & & -7.5 & -0.25 & 15 & 2 \\
\hline 5 & -3.5 & & & -3 & -0.25 & 118 & 2 \\
\hline 6 & -5.75 & -2.25 & 15 & -4.75 & -2.75 & 170 & 3 \\
\hline 7 & 0.25 & & & 0.75 & -0.25 & 180 & 1 \\
\hline 8 & -4 & -0.25 & 15 & -3.75 & -0.5 & 160 & 2 \\
\hline 9 & -5.25 & -1.5 & 165 & -5.25 & -1.75 & 15 & 3 \\
\hline 10 & 0.75 & -2.25 & 90 & 0.75 & 2.75 & 80 & 4 \\
\hline 11 & -7.25 & & & -6.5 & & & 2 \\
\hline 12 & -3 & -1.5 & 40 & -3 & -1 & 165 & 3 \\
\hline 13 & -0.25 & & & -0.75 & & & 1 \\
\hline 14 & 0 & & & 0 & & & 1 \\
\hline 15 & -1 & -4.25 & 8 & -1 & -4.25 & 8 & 3 \\
\hline 16 & 0.25 & & & 0.25 & -0.75 & 55 & 1 \\
\hline 17 & -3 & -2 & 10 & -0.75 & -2 & 170 & 4 \\
\hline 18 & -3 & -0.25 & 75 & -2.25 & -2 & 110 & 4 \\
\hline 19 & 0 & -1.25 & 160 & 0.25 & -1.25 & 60 & 4 \\
\hline 20 & -6.5 & -0.75 & 40 & -3 & -1 & 130 & 4 \\
\hline
\end{tabular}

\section{Experiment 1}

Experiment one was designed as a digital implementation of a regular eye test, similar to the eye tests used in ophthalmology to asses visual acuity [71]. This experiment was done as a first step in gauging how well the precompensation process works in 
assisting subjects with visual refractive errors to interact more efficiently by measuring their visual acuity before and after precompensation for each eye independently.

A tumbling E test was chosen over other methods of testing visual acuity, such as the Early Treatment Diabetic Retinopathy Study (ETDRS) eye test, to minimize the involvement of cognitive functions, except the recognition of orientation of the $\mathrm{E}$. The tumbling-E are mostly used for visual acuity screenings in children, illiterate persons, or non-English speakers [20]. An example of a tumbling E display is shown in Figure 3-29.

Used in conjunction with an appropriate scoring system, these charts are better suited for research and statistical analysis than the conventional letter charts used in refraction [55], such as the Snellen chart. Bailey and Lovie [71] proposed to measure of visual acuity in terms of "the logarithm of the angular limb width (in minutes of arc) of the smallest letters recognized at 6 meters". This notation is called the 'logMAR', standing for the logarithm of the minimum angle of resolution, and is still used in ophthalmology today [55]. Table 3-6 shows the relationship between the logMAR scale and the size of the strokes (in minutes of arc subtended) for each size of the letter E.

As $\log$ MAR values decrease with letter size, as seen in Table 3-6, the scoring systems and calculation of the corresponding visual acuity can be complicated [55]. The basic problem is related to the difference between the arithmetic mean and the geometric mean for a set of numbers [38]. As shown in Table 3-6, the letters on each line follow a geometric progression. In other words, they change in a uniform step on a logarithmic scale. Thus, any acceptable statistical analysis, such as mean, standard deviation, or 
variance, must be done first using the $\log$ MAR values, and then converting into a visual distance acuity score.

This can be accomplished using the following equations [17]:

$$
\log M A R=-\log (V)
$$

where $\mathrm{V}$ is the decimal acuity.

Table 3-6 The log of the Minimum Angle Resolved (logMAR) scale, corresponding minimum angles of resolution, and equivalent decimal $\mathrm{V}$ and Snellen distance acuities

\begin{tabular}{ccccc}
\hline \hline $\begin{array}{c}\text { logMAR } \\
\text { steps }\end{array}$ & $\begin{array}{c}\text { Angular Size of } \\
\text { detail (in minutes of } \\
\text { arc) }\end{array}$ & $\begin{array}{c}\text { Corresponding distance acuities } \\
\text { Decimal } \\
\text { at }\end{array}$ & $\begin{array}{c}\text { (denominator } \\
\text { of Snellen } \\
\text { Fraction }\end{array}$ & $\begin{array}{c}\text { at 20ft } \\
\text { (denominator } \\
\text { of Snellen } \\
\text { Fraction) }\end{array}$ \\
\hline 1.3 & 20 & 0.05 & 120 & 400 \\
1.2 & 15.9 & 0.063 & 95 & 320 \\
1.1 & 12.7 & 0.079 & 75 & 250 \\
\hline 1 & 10 & 0.1 & 60 & 200 \\
0.9 & 7.9 & 0.126 & 48 & 160 \\
0.8 & 6.3 & 0.158 & 38 & 125 \\
0.7 & 5 & 0.2 & 30 & 100 \\
0.6 & 4 & 0.251 & 24 & 80 \\
0.5 & 3.2 & 0.316 & 19 & 63 \\
0.4 & 2.5 & 0.398 & 15 & 50 \\
0.3 & 2 & 0.501 & 12 & 40 \\
0.2 & 1.6 & 0.631 & 9.5 & 32 \\
0.1 & 1.3 & 0.794 & 7.5 & 25 \\
0 & 1 & 1 & 6 & 20 \\
\hline-0.1 & 0.8 & 1.259 & 4.75 & 16 \\
-0.2 & 0.6 & 1.585 & 3.75 & 12.5 \\
-0.3 & 0.5 & 1.995 & 3 & 10 \\
\hline \hline
\end{tabular}

This decimal acuity can be converted back into the Snellen equivalent by

$$
\text { denominator of Snellen }=20 / \mathrm{V} \text {. }
$$


The score for an eye exam is usually found by number of letters the person read correctly. Although recording the last line that was completely read, or if the person reads at least up to a threshold, three out of the five letters counting as a complete line, is acceptable, it tends to reduce the precision measurement [38].

Thus, a more accurate way of computing the visual acuity score is as follows:

$$
t L V=c L V-0.1 \cdot(n / 5)
$$

where $t L V$ is the true $\operatorname{logMAR}$ value, $c L V$ is the $\log$ MAR value for the last line completely read, and $n$ is the number of letters read correctly on the subsequent line. For the purposes of this dissertation, a line is considered completely read if the subject correctly states the orientation of the first presentation of the letter $\mathrm{E}$ for that line. Equation 3-43 is only used when the subject incorrectly states the orientation of the current line.

The experiment was created in MATLAB $^{\circledR}$ using its GUI capabilities. An example of the GUI interface for testing is show in Figure 3-29. For this test, each subject was positioned approximately $50 \mathrm{~cm}$ from the screen. An eye patch was supplied to the subjects so they could perform the test monocularly. The test began by presenting the subject with an uncompensated letter $\mathrm{E}$, in a random orientation at the largest size, corresponding to a visual acuity of $\log M A R=1.0$. The subject was then asked to identify the orientation of the letter, i.e. whether the opening is pointing to the right, left, up or down. If the subject correctly identified the letter, the next smaller acuity level is presented and a "correct" score is recorded. Whenever the first letter (of each line) was 
identified incorrectly by the subject, the acuity level was increased by one and the entire line was tested.

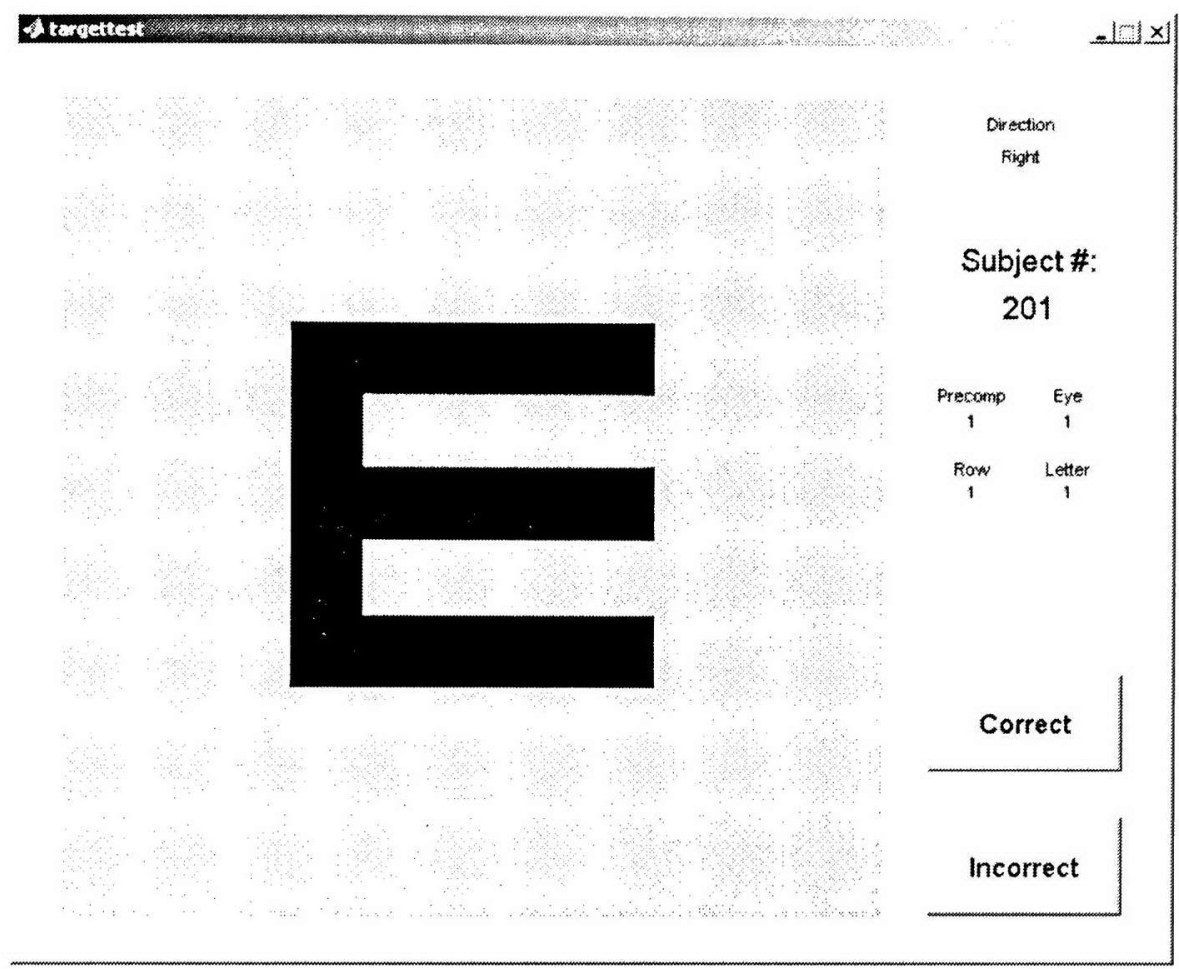

Figure 3-29 Example GUI interface for Human subject VA test

If the subject identified the very first letter incorrectly, that line was tested and this portion of the test was completed. If the subject reached the smallest line (corresponding to a $\log \mathrm{MAR}=0$ ), that entire line was tested, and then this portion of the test was complete. The same test was then applied using precompensated versions of the letter E. Once the uncompensated and precompensated tests were complete, the remaining eye was tested. The order of eyes tested was right eye first, then left eye.

Thus, the experiment design is organized as follows: Three factors were studied: "Group", which represents one of the four groups classifying the subjects, "Method", which indicates whether or not the images to be viewed by the subject were 
precompensated, and "Eye", which represents either the right or the left eye. The "Group" factor, G, consists of four levels, each level corresponding to one of the four groups: controls, myopic, myopic-astigmatic, or keratoconic. The "Method" factor, M, consists of two levels, the original letter $\mathrm{E}$ (level one) and the precompensated letter $\mathrm{E}$ (level 2). The experiment can be considered a repeated measures experiment with three fixed factors: Group (G) - four levels, Eye (E) - two levels, and Method (M) - two levels. Every treatment combination was applied to twenty subjects. The dependent variable, based on the output of each trial, is the visual acuity score as described by Equation 3-43. The experiment is treated as randomized complete block design (RCBD) experiment, taking subjects as a random factor and blocking on it. The model for the analysis of variance is as follows:

$$
Y_{i j k m}=\mu+G_{i}+L_{m(i)}+E_{j}+G E_{i j}+M_{k}+G M_{i k}+E M_{j k}+G E M_{i j k}+\varepsilon_{i j k m}
$$

with the subject factor nested within the group factor. The factors are summarized in Table 3-7.

Table 3-7 Factors for human subjects experiment 1

\begin{tabular}{ccc} 
Fixed Factors & Variable & Range \\
\hline Group & $G_{i}$ & $i=1: 4$ \\
Eye & $E_{j}$ & $j=1: 2$ \\
Method & $M_{k}$ & $k=1: 2$ \\
Random Factor & & \\
\cline { 1 - 1 } Subject & $P_{m}$ & $m=1: 20$ \\
\hline
\end{tabular}

\section{Experiment 2}

Experiment two was designed to asses the improvement that the precompensation processing delivers to subjects for recognizing a target icon from a group of icons of 
various sizes. It was designed using $\mathrm{MATLAB}^{\circledR}$, s GUI tools. Figure 3-30 and Figure 3-31 show examples of the stimulus and icon screens.

Each subject was positioned approximately $50 \mathrm{~cm}$ from the screen. An eye patch was supplied to the subject so they could perform the test monocularly. The test began by presenting the subject with a stimulus screen consisting of a large icon, with the maximum size being approximately $59 \mathrm{~mm}$ wide (e.g. Figure 3-30), uncompensated. The initial stimulus icon was selected at random from a pool of six different Microsoft Windows icons (save, print, briefcase, binoculars, folder, and image).
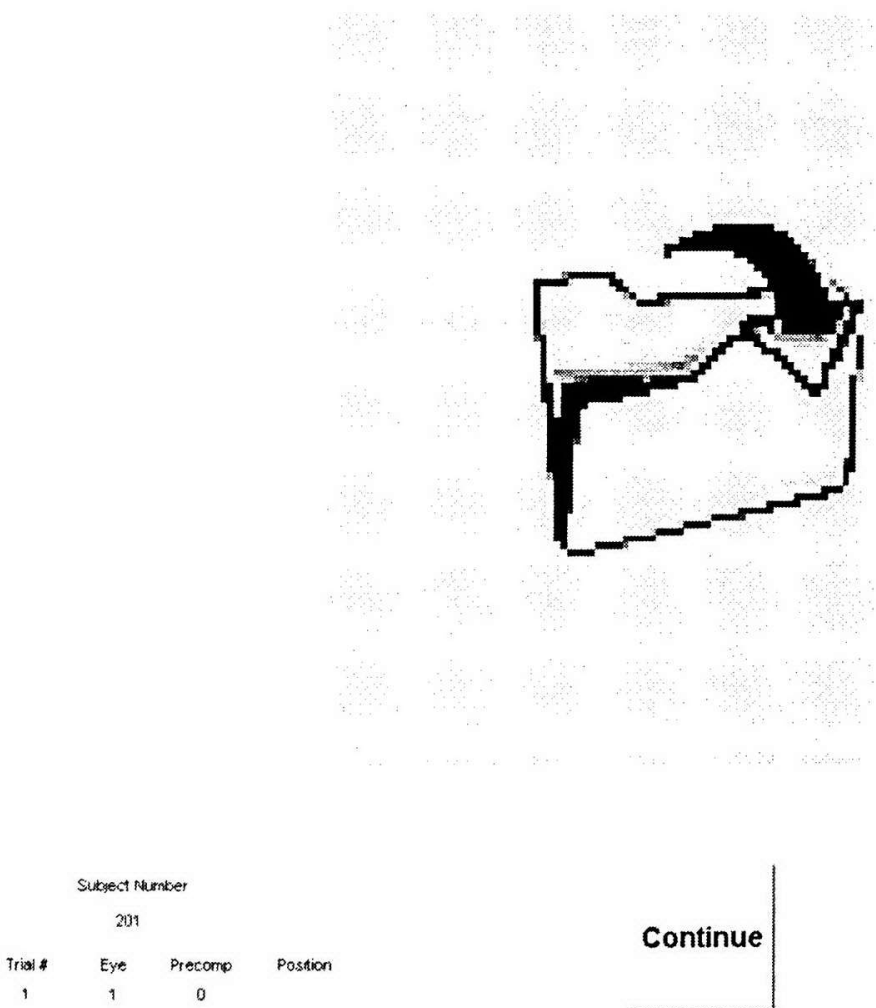

Figure 3-30 Stimulus Screens for the Icon Test 


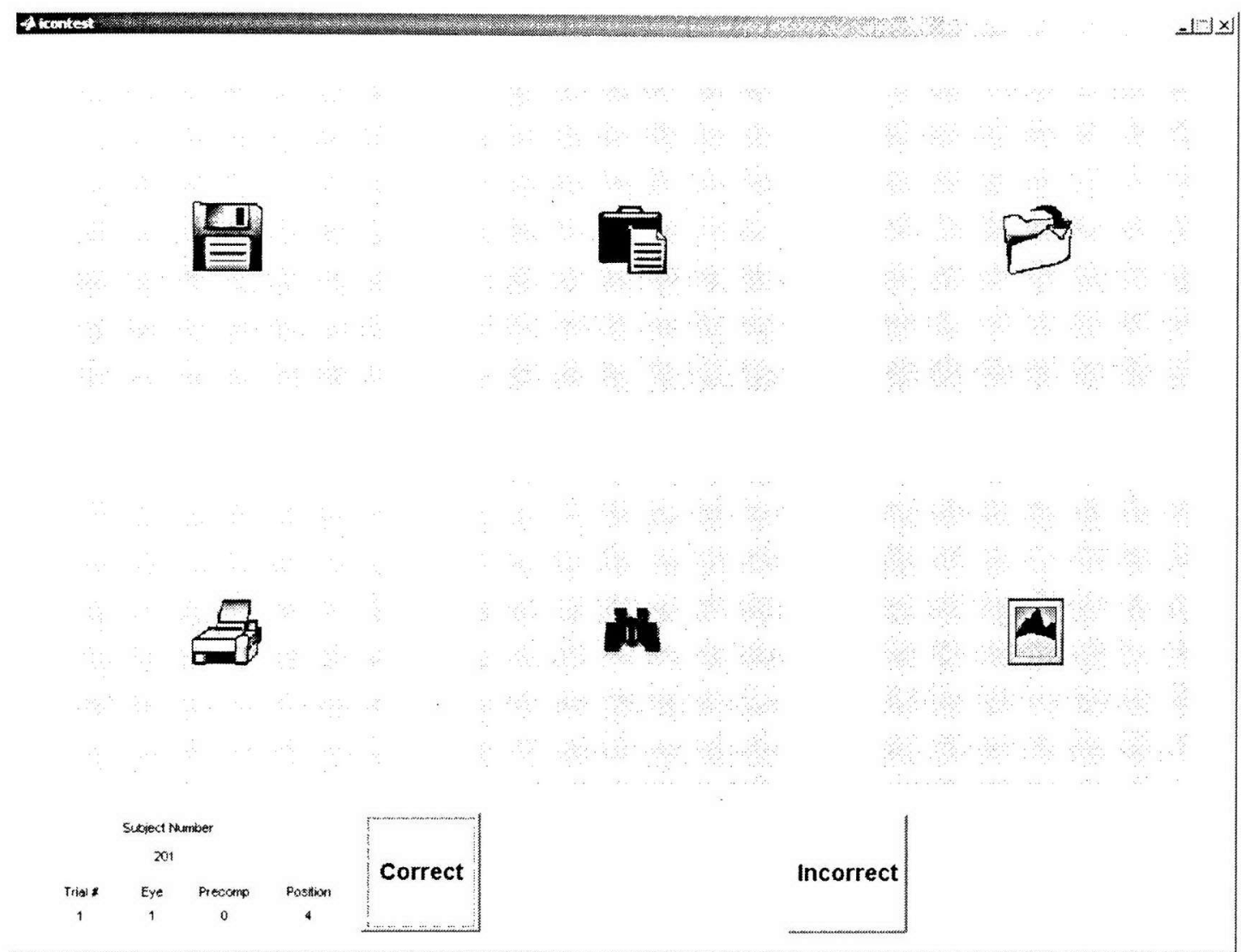

Figure 3-31 Target Screens for the Icon Test

The subject was then asked to respond when he/she was ready for the target icons to be displayed. Each of the six icons was displayed in a $2 \times 3$ array. The position and size of each icon was selected at random for each treatment level. The size of each icon could vary from $15 \mathrm{~mm}$ to $38 \mathrm{~mm}$ wide. The subject was then asked to identify the icon that matched the large stimulus icon. The answer for each target screen was recorded as a "correct" or "incorrect" identification of the icon. Once the subject was done with the target screen, another large icon was presented and the sequence began again. Each icon position and size combination was tested twice. This equates to thirty six trials for this portion of the test, i.e., uncompensated icons. The test was then repeated using precompensated stimulus and target icons. Once the uncompensated and precompensated 
tests were complete, the remaining eye was tested. The order of eyes tested was right eye first, then left eye.

Thus, the experiment can be considered a repeated measures experiment with four fixed factors: Group (G) - four levels, Eye (E) - two levels, Size (S) - three levels, and Method (M) - two levels. Every treatment combination was applied to the twenty subjects in a randomized order. The dependent variable is the number of correct icons per size, for each treatment combination. The experiment is treated as randomized complete block design (RCBD) experiment, taking subjects as a random factor and blocking on it. The model for the analysis of variance is as follows:

$$
\begin{aligned}
& Y_{i j k m}=\mu+G_{i}+P_{n(i)}+E_{j}+G E_{i j}+S_{k}+G S_{i k}+E S_{j k}+G E S_{i j k}+M_{m}+ \\
& G M_{i m}+E M_{j m}+G E M_{i j m}+S M_{k m}+G S M_{i k m}+E S M_{j k m}+G E S M i_{i j k m}+\varepsilon_{i j k m n}
\end{aligned}
$$

with subjects nested in groups.

The factors are summarized in Table 3-8.

Table 3-8 Factors for human subject experiment 2

\begin{tabular}{ccc} 
Fixed Factors & Variable & Range \\
\hline Group & $G_{i}$ & $i=1: 4$ \\
Eye & $E_{j}$ & $j=1: 2$ \\
Size & $S_{k}$ & $k=1: 3$ \\
Method & $M_{m}$ & $m=1: 2$ \\
Random Factor & & \\
\cline { 1 - 1 } Subject & $P_{n}$ & $n=1: 20$ \\
\hline
\end{tabular}

\subsection{Data Analysis Methods}

The data acquired from both the software simulation and the artificial eye, with and without precompensation, were analyzed by using mixed design analysis of variance 
(ANOVA). A mixed design ANOVA was applied to the data for each experiment to detect the effect that treatments have on image similarity by observing any statistically significance of the factors and their interactions. The data was found to be normal and these analyses were accompanied by orthogonal contrasts of the various factors.

A nested factorial mixed ANOVA was used for experiment one and two of the human subject tests. Although the number of subjects per group was low ( $\mathrm{n}=5$ subjects per group) it was found that the data satisfied the assumptions required for parametric analysis based on testing the Studentized residuals for outliers, normality, and testing the homogeneity of variance between cells. 


\section{CHAPTER 4}

\section{RESULTS}

This chapter presents the results obtained from the evaluation of the proposed method of precompensation based on the experiments designed to quantify the improvement on visual acuity and icon identification tasks, as described in Chapter 3. Data gathered from the experiments and the statistical analyses performed on these data are provided. The discussion and interpretation of the results are presented in Chapter 5.

\subsection{Software Simulation of the Entire Process}

Initially, the entire physical process of sight was simulated in software to provide a sense of the performance of the precompensation algorithm towards its application on digital images of icons presented on an LCD panel. The process of obtaining a simulated retinal representation of any image shown to a user is outlined in chapter three. Simulations of the retinal images of various icons used in subsequent tests are shown in Figure 4-1.

The simulated retinal images for a normal and precompensated image are shown, as well as the PSF used in the simulation and the original icon. These images were generated with $\mathrm{k}=0.005$ in the deconvolution step, Equation 3-6. After the precompensated images were generated, they were post-processed using ContrastLimited Adaptive Histogram Equalization using the MATLAB ${ }^{\circledR}$ function, adapthisteq with the default parameters. The reader is referred to [46] for a detailed description of adaptive histogram equalization. 
As can be seen from Figure 4-1, the retinal images are more clearly defined for the precompensated images as compared to the normal images, although the background gray level appears changed and contrast has been reduced.

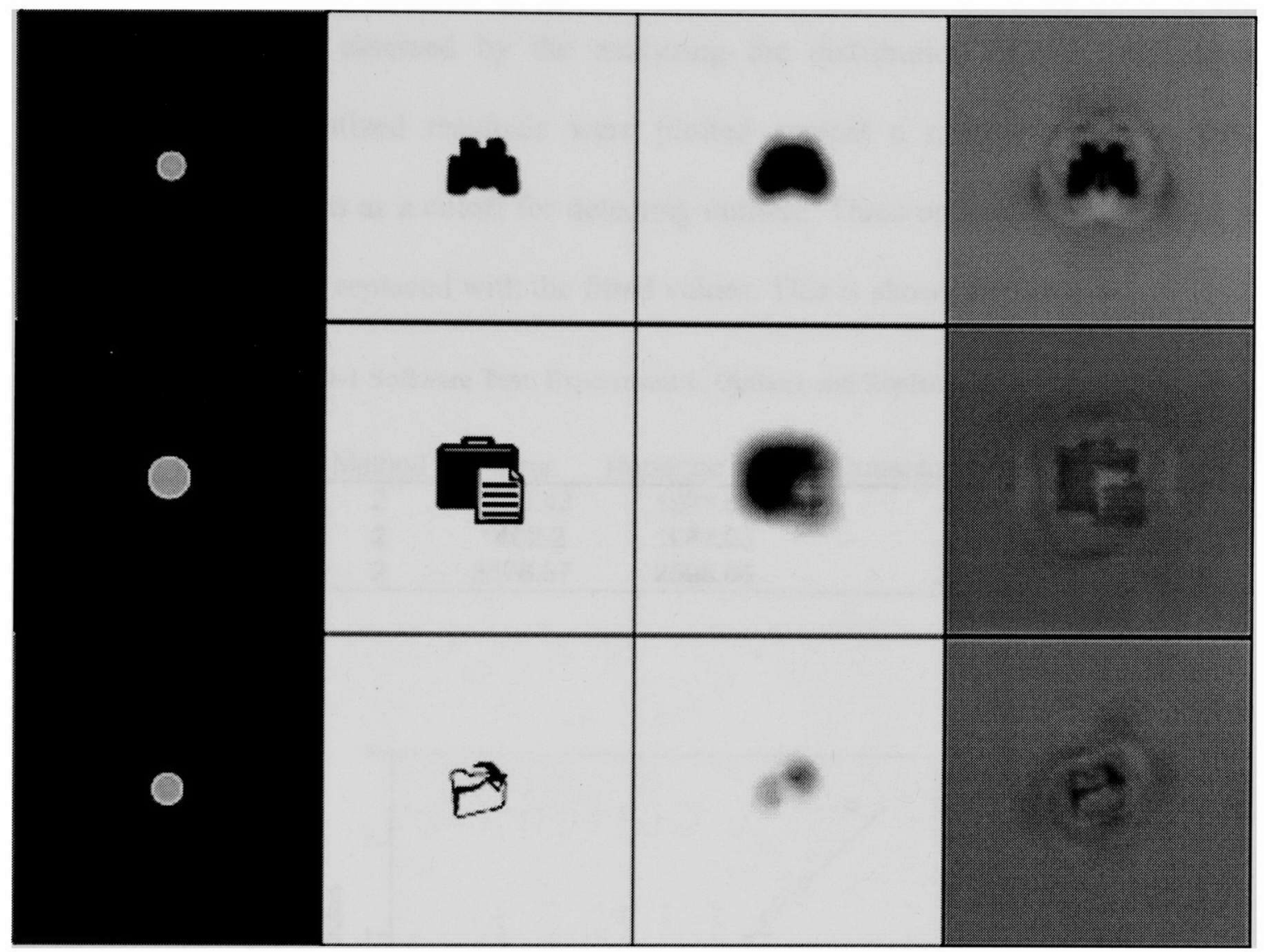

Figure 4-1 Three icons with retinal image simulation of normal and precompensated versions - Column one contains the PSF used to in precompensation and simulated viewing, column two contains the original icon, column three is the simulated retinal image of the icon, and column four is the simulated retinal image of the precompensation

\subsubsection{Experiment 1}

Experiment one for the software test is a factorial experiment blocking on the icon factor. The data collected for this experiment consists of four columns of data, each representing a treatment combination on thirty-six unique subjects. SPSS ${ }^{\circledR}$ was used to 
generate a statistical analysis. The data was first screened for outliers, normality and homogeneity of variance.

\section{$\underline{\text { Outliers }}$}

Outliers were assessed by the analyzing the distribution of the Studentized residuals. The Studentized residuals were plotted against a $t_{9}$ distribution. A $95 \%$ confidence interval was as a cutoff for detecting outliers. Three outliers were detected in the data and they were replaced with the fitted values. This is shown in Table 4-1.

Table 4-1 Software Test: Experiment 1, Outliers and Replacements

\begin{tabular}{ccccccc} 
Icon & Size & Method & Error & Replaced Value & Studentized Residual & T Quartile \\
\hline 6 & 3 & 2 & 1220.43 & 1923.82 & -2.5 & 0.01 \\
1 & 3 & 2 & 1402.2 & 1967.63 & -2.01 & 0.02 \\
4 & 3 & 2 & 3178.37 & 2596.66 & 2.07 & 0.98 \\
\hline
\end{tabular}

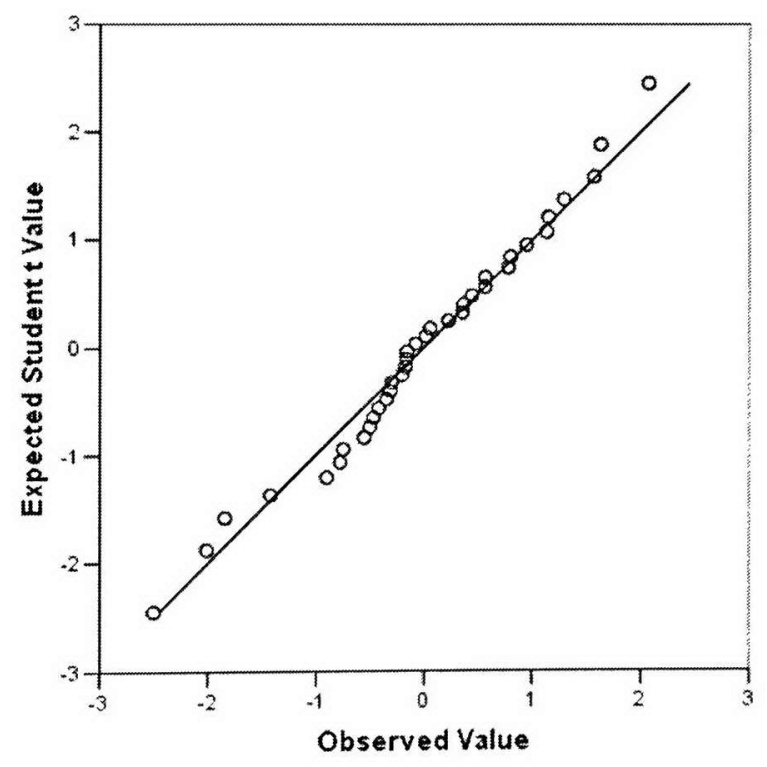

Figure 4-2 Software Test: Experiment 1, T Quantile Plot of Studentized Residuals 
Normality

Normality was assessed by testing the normality of the Studentized residuals. One of the assumptions of the ANOVA is that the distribution of the residuals is normal. A Tquantile plot $(\mathrm{DF}=9)$, a histogram, and both the Kolmogorov-Smirnov and Shapiro-Wilk test were applied to the data. The residuals of the data are normal, with a $p=0.777$ for the Shapiro-Wilk test. Figure 4-2, Figure 4-3, and Table 4-2 summarize these results.

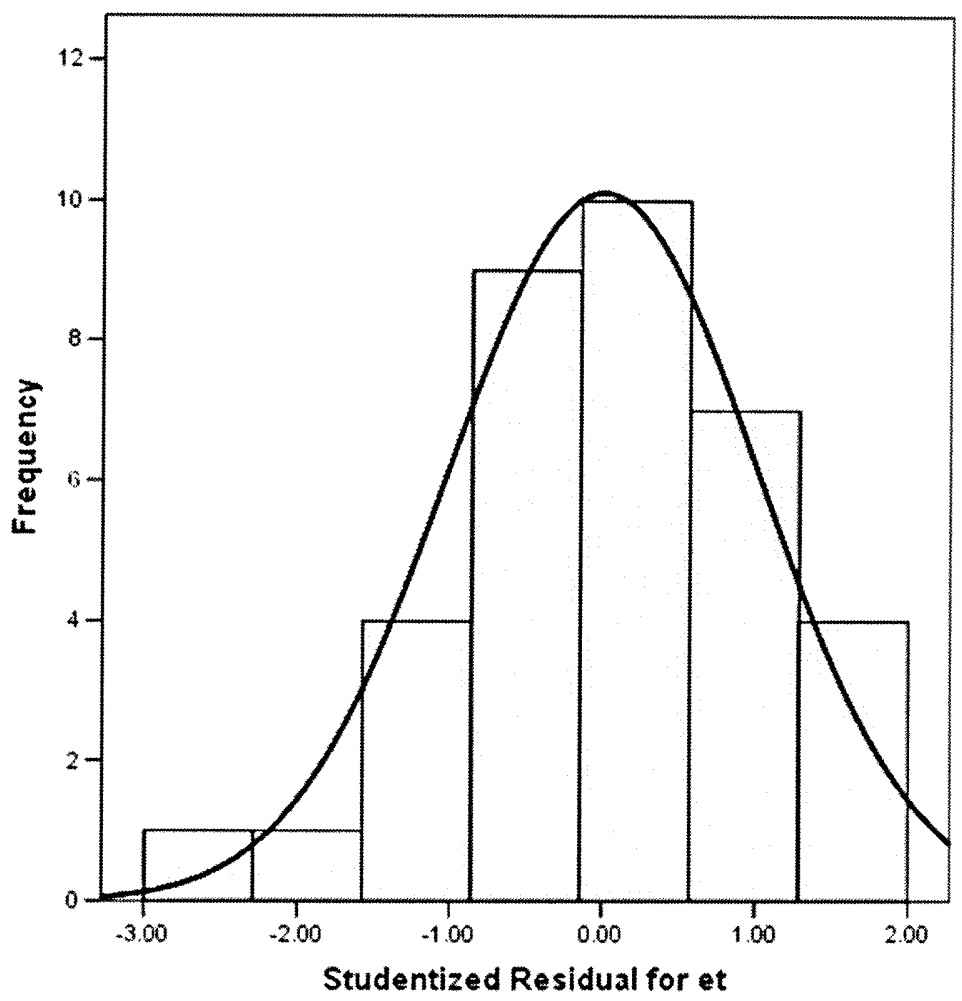

Figure 4-3 Software Test: Experiment 1, Histogram of Studentized Residuals with Normal Curve

Table 4-2 Software Test: Experiment 1, Normality Test on Studentized Residuals

\begin{tabular}{|l|r|r|r|r|r|r|}
\hline & \multicolumn{3}{|c|}{ Kolmogorov-Smirnov $^{\mathrm{a}}$} & \multicolumn{3}{c|}{ Shapiro-Wilk } \\
\cline { 2 - 7 } & Statistic & \multicolumn{1}{c|}{$\mathrm{df}$} & \multicolumn{1}{c|}{ Sig. } & Statistic & \multicolumn{1}{c|}{ df } & Sig. \\
\hline $\begin{array}{l}\text { Studentized } \\
\text { Residual for et }\end{array}$ & .092 & 36 & $.200^{*}$ & .981 & 36 & .777 \\
\hline
\end{tabular}




\section{Homogeneity of Variance}

The homogeneity of variance was tested by applying Levene's statistic to the distribution of each cell of the model [28]. Initially, the homogeneity of variance assumption was not met. However, after applying a power transformation of $y=x^{b}$, where $\mathrm{x}$ is the original data, $\mathrm{y}$ is the result of the transformation, and $\mathrm{b}$ is 0.118 (as suggested by the Spread vs. Level plot in SPSS ${ }^{\circledR}$ ), the assumption of equal variances was met. The results of the Levene test are shown in Table 4-3.

Table 4-3 Software Test: Experiment 1, Levene's Test for homogeneity of cell variances

\begin{tabular}{|c|c|c|c|c|c|}
\hline & & $\begin{array}{l}\text { Levene } \\
\text { Statistic }\end{array}$ & df1 & df2 & Sig. \\
\hline \multirow[t]{4}{*}{ et2 } & Based on Mean & .430 & 5 & 30 & .824 \\
\hline & Based on Median & .180 & 5 & 30 & .968 \\
\hline & $\begin{array}{l}\text { Based on Median and } \\
\text { with adjusted df }\end{array}$ & .180 & 5 & 25.314 & .967 \\
\hline & Based on trimmed mean & .402 & 5 & 30 & .844 \\
\hline
\end{tabular}

\section{ANOVA}

Upon verification of the required assumptions, an ANOVA was applied to the data. The results obtained are shown in Table 4-4. The factor of size and method were significant, with $\mathrm{p}<0.005$. No interactions were present. The results of the difference contrasts on size and method are shown in Table 4-5 and Table 4-6, respectively. The estimated marginal mean for the size and method factors are shown in Table 4-7 and Table 4-8. The Student-Newman Keuls test was applied to size and the results are show in Table 4-9. 
Table 4-4 ANOVA Table for Software Test: Experiment 1

\begin{tabular}{|l|r|r|r|r|r|}
\hline Source & $\begin{array}{c}\text { Type III Sum } \\
\text { of Squares }\end{array}$ & df & Mean Square & \multicolumn{1}{|c|}{ F } & \multicolumn{1}{c|}{ Sig. } \\
\hline Corrected Model & $8.761^{a}$ & 10 & .876 & 35.412 & .000 \\
Intercept & 474.187 & 1 & 474.187 & 19166.581 & .000 \\
icon & 2.141 & 5 & .428 & 17.308 & .000 \\
size & 5.466 & 2 & 2.733 & 110.458 & .000 \\
method & 1.083 & 1 & 1.083 & 43.790 & .000 \\
size* method & .071 & 2 & .036 & 1.436 & .257 \\
Error & .619 & 25 & .025 & & \\
Total & 483.567 & 36 & & & \\
Corrected Total & 9.379 & 35 & & & \\
\hline
\end{tabular}

Table 4-5 Software Test: Experiment 1 Contrast on Size

\begin{tabular}{|c|c|c|c|}
\hline \multirow{2}{*}{\multicolumn{3}{|c|}{ size Difference Contrast }} & $\begin{array}{c}\text { Depende } \\
\text { nt } \\
\text { Variable }\end{array}$ \\
\hline & & & et2 \\
\hline \multirow[t]{7}{*}{ Level 2 vs. Level 1} & \multicolumn{2}{|l|}{ Contrast Estimate } & .387 \\
\hline & \multicolumn{2}{|l|}{ Hypothesized Value } & 0 \\
\hline & \multicolumn{2}{|c|}{ Difference (Estimate - Hypothesized) } & .387 \\
\hline & \multicolumn{2}{|l|}{ Std. Error } & .064 \\
\hline & \multicolumn{2}{|l|}{ Sig. } & .000 \\
\hline & \multirow{2}{*}{$\begin{array}{l}95 \% \text { Confidence Interval } \\
\text { for Difference }\end{array}$} & Lower Bound & .255 \\
\hline & & Upper Bound & .519 \\
\hline \multirow[t]{7}{*}{ Level 3 vs. Previous } & \multirow{3}{*}{\multicolumn{2}{|c|}{$\begin{array}{l}\text { Contrast Estimate } \\
\text { Hypothesized Value } \\
\text { Difference (Estimate - Hypothesized) }\end{array}$}} & .756 \\
\hline & & & 0 \\
\hline & & & .756 \\
\hline & \multicolumn{2}{|l|}{ Std. Error } & .056 \\
\hline & \multicolumn{2}{|l|}{ Sig. } & .000 \\
\hline & \multirow{2}{*}{$\begin{array}{l}95 \% \text { Confidence Interval } \\
\text { for Difference }\end{array}$} & Lower Bound & .641 \\
\hline & & Upper Bound & .870 \\
\hline
\end{tabular}


Table 4-6 Software Test: Experiment 1 Contrast on Method

\begin{tabular}{|l|r|r|}
\hline method Difference & \multicolumn{1}{c|}{$\begin{array}{c}\text { Depende } \\
\text { nt } \\
\text { Contrast }\end{array}$} \\
\cline { 2 - 3 } & \multicolumn{1}{|c|}{ et2 } \\
\hline Level 2 vs. Level 1 & $\begin{array}{l}\text { Contrast Estimate } \\
\text { Hypothesized Value }\end{array}$ & .347 \\
& Difference (Estimate - Hypothesized) & 0 \\
& Std. Error & .347 \\
& Sig. & .052 \\
& $95 \%$ Confidence Interval & .000 \\
& for Difference & .239 \\
& Upwer Bound & .455 \\
\hline
\end{tabular}

Table 4-7 Software Test: Experiment 1, Estimated Marginal Mean for size

\begin{tabular}{|l|r|r|r|r|}
\hline & & & \multicolumn{2}{|c|}{$95 \%$ Confidence Interval } \\
\cline { 4 - 5 } size & Mean & Std. Error & Lower Bound & Upper Bound \\
\hline 1.00 & 3.184 & .045 & 3.091 & 3.278 \\
2.00 & 3.571 & .045 & 3.477 & 3.664 \\
3.00 & 4.133 & .045 & 4.040 & 4.227 \\
\hline
\end{tabular}

Table 4-8 Software Test: Experiment 1, Estimated Marginal Mean for method

\begin{tabular}{|l|r|r|r|r|}
\hline & & & \multicolumn{2}{|c|}{$95 \%$ Confidence Interval } \\
\cline { 4 - 5 } method & Mean & Std. Error & Lower Bound & Upper Bound \\
\hline 1.00 & 3.456 & .037 & 3.379 & 3.532 \\
2.00 & 3.803 & .037 & 3.726 & 3.879 \\
\hline
\end{tabular}

Table 4-9 Software Test: Experiment 1, S-N-K for size

\begin{tabular}{|l|r|r|r|r|}
\hline & & \multicolumn{3}{|c|}{ Subset } \\
\cline { 3 - 5 } size & $\mathrm{N}$ & 1 & \multicolumn{1}{|c|}{2} & \multicolumn{1}{c|}{3} \\
\hline 1.00 & 12 & 3.1840 & & \\
2.00 & 12 & & 3.5708 & \\
3.00 & 12 & & & 4.1331 \\
Sig. & & 1.000 & 1.000 & 1.000 \\
\hline
\end{tabular}




\section{$\underline{\text { Key Findings }}$}

The factors size and method showed significant effects on the mean of the Difference Measure. Additionally, the S-N-K test revealed that for the size factor, each size was significantly different from every other size, which is reinforced by the difference contrast on size, shown in Table 4-5. The levels of size correspond to increasingly larger icons, which points to the fact that the larger the icon, the larger the error measure should be because the measure itself is not normalized. Similarly, the error measure for level one of the method factor is significantly lower than for level two, indicating the precompensation algorithm was successful in lowering the error measure as compared to the same measure without precompensation.

\subsubsection{Experiment 2}

Experiment two for the software test is a repeated measures design that is treated as a $\mathrm{RBCD}$, blocking on subjects, which is a random factor. The data collected for this experiment consists of five columns of data, each representing a treatment combination on twenty unique subjects. SPSS ${ }^{\circledR}$ was used to generate a statistical analysis. The data was first screened for outliers, normality and homogeneity of variance. After removing the outliers, the first pass of the data screening revealed that the data was not normal. A constant of 300 was first added to the data, followed by a 3/4th root transformation. The transformed data was then screened for normality and homogeneity of variance. The results are as follows. 


\section{$\underline{\text { Outliers }}$}

Outliers were assessed by analyzing the distribution of the Studentized residuals. The Studentized residuals were plotted against a $t_{189}$ distribution. A $95 \%$ confidence interval was as a cutoff for detecting outliers. Thirty-Two outliers were detected in the data and they were replaced with the fitted values. These outliers represent $4.4 \%$ of the data. This is shown in Table 4-10.

Table 4-10 Software Test: Experiment 2, Outliers

\begin{tabular}{|c|c|c|c|c|c|}
\hline Icon & Size & Method & Subject & Error & Replaced Value \\
\hline 6 & 3 & 2 & 8 & 395.55 & 1214.86 \\
\hline 5 & 3 & 2 & 8 & 2293.29 & 3055.73 \\
\hline 4 & 3 & 2 & 8 & 2055.71 & 2762.82 \\
\hline 2 & 3 & 2 & 8 & 1950.07 & 2627.44 \\
\hline 3 & 3 & 2 & 8 & 2341.58 & 2929.88 \\
\hline 5 & 2 & 2 & 8 & 973.38 & 1466.31 \\
\hline 1 & 3 & 2 & 8 & 1003.78 & 1484.55 \\
\hline 5 & 2 & 2 & 2 & 1192.05 & 1641.67 \\
\hline 6 & 3 & 2 & 15 & 926.64 & 1371.58 \\
\hline 5 & 2 & 2 & 15 & 1199.36 & 1623.03 \\
\hline 3 & 3 & 1 & 18 & 1647.65 & 2025.47 \\
\hline 3 & 3 & 1 & 13 & 1644.29 & 2018.31 \\
\hline 3 & 3 & 1 & 17 & 1670.74 & 2034.85 \\
\hline 5 & 3 & 2 & 2 & 2881.77 & 3231.1 \\
\hline 5 & 1 & 2 & 8 & 305.58 & 654.42 \\
\hline 2 & 3 & 2 & 2 & 2481.57 & 2802.81 \\
\hline 4 & 2 & 2 & 2 & 838.37 & 1153.27 \\
\hline 3 & 2 & 1 & 8 & 1356.26 & 725.26 \\
\hline 4 & 2 & 1 & 8 & 976.22 & 475.47 \\
\hline 3 & 1 & 1 & 8 & 581.63 & 147.01 \\
\hline 5 & 2 & 1 & 8 & 1088.14 & 653.76 \\
\hline 1 & 2 & 1 & 8 & 465.96 & 32.81 \\
\hline 4 & 1 & 1 & 8 & 430.01 & -2.94 \\
\hline 1 & 3 & 1 & 2 & 1334.25 & 905.38 \\
\hline 3 & 2 & 1 & 2 & 1302.84 & 900.63 \\
\hline 5 & 3 & 2 & 1 & 3901.11 & 3517.43 \\
\hline 1 & 1 & 1 & 8 & 178.19 & -198.15 \\
\hline 6 & 1 & 1 & 8 & 267.59 & -96.4 \\
\hline 1 & 3 & 1 & 8 & 1078.14 & 730.01 \\
\hline 4 & 3 & 1 & 3 & 2154.58 & 1812.88 \\
\hline 2 & 1 & 1 & 8 & 283.5 & -28.93 \\
\hline
\end{tabular}


Normality was assessed by testing the normality of the Studentized residuals. One of the assumptions of the ANOVA is that the distribution of the residuals is normal. A Tquantile plot $(\mathrm{DF}=189)$, a histogram, and both skewness and kurtosis values were obtained for the data. The residuals of the data are approximately normal for the histogram and quantile plots. The skewness and kurtosis values do not indicate any strong deviation from normality. Figure 4-4, Figure 4-5, Table 4-11 summarize these results.

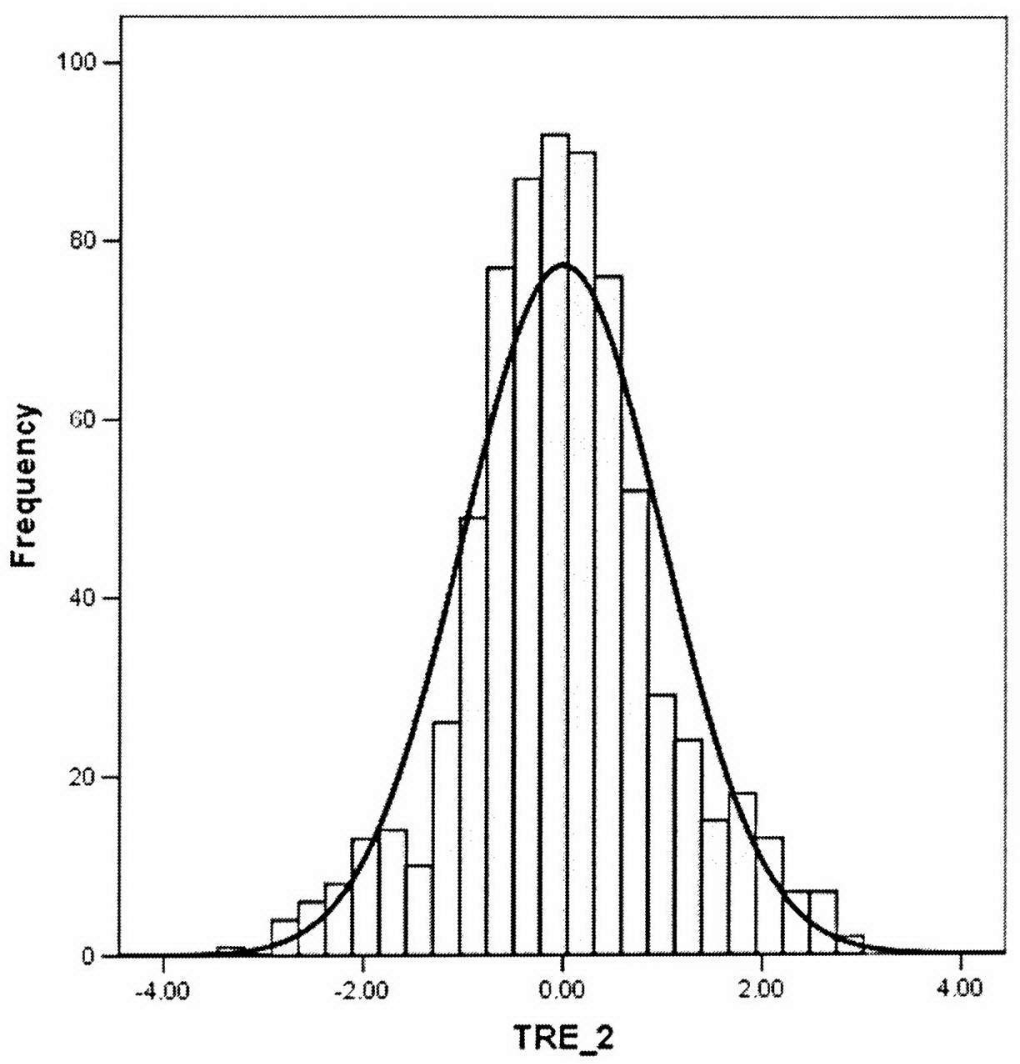

Figure 4-4 Software Test: Experiment 2, Histogram of residuals 


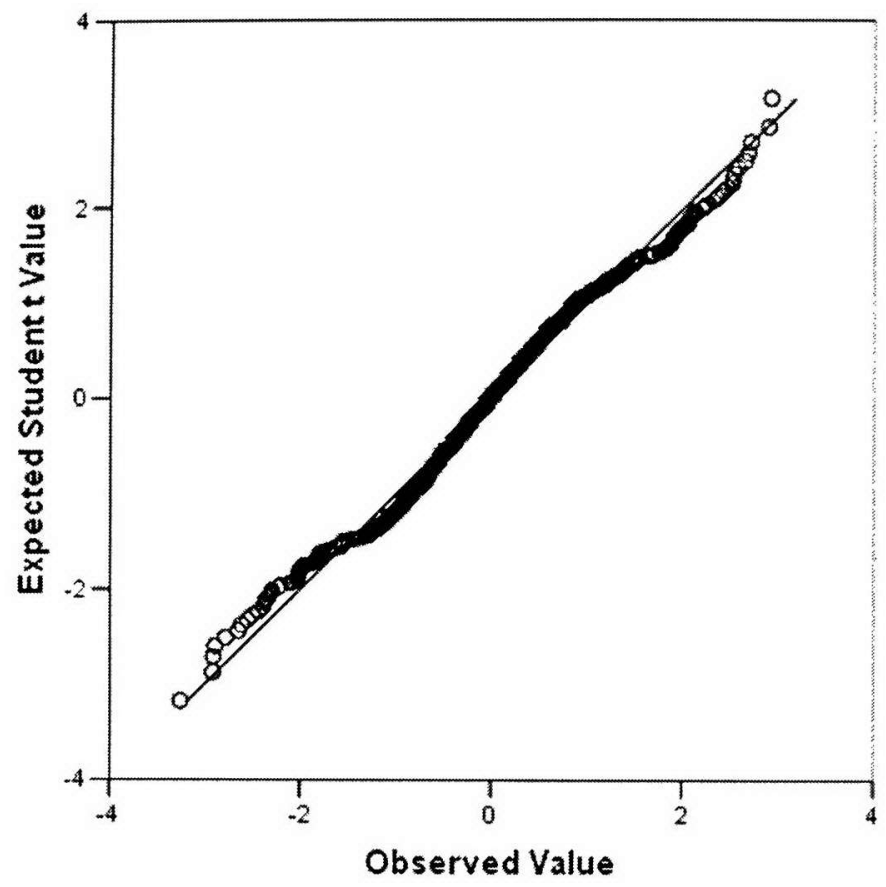

Figure 4-5 Software Test: Experiment 2, T-quantile plot of residuals

Table 4-11 Software Test: Experiment 2, Skewness and Kurtosis of residuals

\begin{tabular}{|l|r|r|r|r|r|}
\hline & \multicolumn{1}{|c|}{$\mathrm{N}$} & \multicolumn{2}{|c|}{ Skewness } & \multicolumn{2}{c|}{ Kurtosis } \\
\cline { 2 - 6 } & Statistic & \multicolumn{1}{c|}{ Statistic } & \multicolumn{1}{c|}{ Std. Error } & Statistic & Std. Error \\
\hline TRE_2 & 720 & .016 & .091 & .645 & .182 \\
Valid N (listwise) & 720 & & & & \\
\hline
\end{tabular}

\section{Homogeneity of Variance}

The homogeneity of variance was tested by applying Levene's statistic to the distribution of each cell of the model. The assumption of equal variances was not met and no transformation was found that would make the variances equal. This is acceptable because the ANOVA with equal sample sizes has been found to be robust against these violations [5]. The results of the Levene test are shown in Table 4-12. 
Table 4-12 Software Test: Experiment 2, Levene's test of homogeneity of cell variances

\begin{tabular}{|c|c|c|c|c|c|}
\hline & & $\begin{array}{l}\text { Levene } \\
\text { Statistic }\end{array}$ & $\mathrm{df1}$ & $\mathrm{df} 2$ & Sig. \\
\hline \multirow[t]{4}{*}{ ErrorNOT } & Based on Mean & 6.808 & 35 & 684 & .000 \\
\hline & Based on Median & 5.245 & 35 & 684 & .000 \\
\hline & $\begin{array}{l}\text { Based on Median and } \\
\text { with adjusted df }\end{array}$ & 5.245 & 35 & 563.852 & .000 \\
\hline & Based on trimmed mean & 6.600 & 35 & 684 & .000 \\
\hline
\end{tabular}

Table 4-13 Software Test: Experiment 2, ANOVA Table

\begin{tabular}{|c|c|c|c|c|c|c|}
\hline Source & & $\begin{array}{l}\text { Type III Sum } \\
\text { of Squares }\end{array}$ & df & Mean Square & $F$ & Sig. \\
\hline \multirow[t]{2}{*}{ Intercept } & Hypothesis & 40527710.3 & 1 & 40527710.33 & 5095.840 & .000 \\
\hline & Error & 151108.836 & 19 & $7953.097^{a}$ & & \\
\hline \multirow[t]{2}{*}{ Icon } & Hypothesis & 1271024.683 & 5 & 254204.937 & 1283.286 & .000 \\
\hline & Error & 131729.223 & 665 & $198.089^{b}$ & & \\
\hline \multirow[t]{2}{*}{ Size } & Hypothesis & 4585849.311 & 2 & 2292924.656 & 11575.221 & .000 \\
\hline & Error & 131729.223 & 665 & $198.089^{b}$ & & \\
\hline \multirow[t]{2}{*}{ Method } & Hypothesis & 1347202.827 & 1 & 1347202.827 & 6800.996 & .000 \\
\hline & Error & 131729.223 & 665 & $198.089^{b}$ & & \\
\hline \multirow[t]{2}{*}{ Subjects } & Hypothesis & 151108.836 & 19 & 7953.097 & 40.149 & .000 \\
\hline & Error & 131729.223 & 665 & $198.089^{b}$ & & \\
\hline \multirow[t]{2}{*}{ Icon * Size } & Hypothesis & 228054.381 & 10 & 22805.438 & 115.127 & .000 \\
\hline & Error & 131729.223 & 665 & $198.089^{b}$ & & \\
\hline \multirow[t]{2}{*}{ Icon * Method } & Hypothesis & 61580.519 & 5 & 12316.104 & 62.175 & .000 \\
\hline & Error & 131729.223 & 665 & $198.089^{b}$ & & \\
\hline \multirow[t]{2}{*}{ Size * Method } & Hypothesis & 133694.968 & 2 & 66847.484 & 337.462 & .000 \\
\hline & Error & 131729.223 & 665 & $198.089^{b}$ & & \\
\hline \multirow{2}{*}{$\begin{array}{l}\text { Icon * Size * } \\
\text { Method }\end{array}$} & Hypothesis & 10274.481 & 10 & 1027.448 & 5.187 & .000 \\
\hline & Error & 131729.223 & 665 & $198.089^{b}$ & & \\
\hline
\end{tabular}

\section{ANOVA}

Upon verification of the required assumptions, an ANOVA was applied to the data. The results of which are shown in Table 4-13. The results of the contrasts on icon, size, and method are shown in Table 4-14, Table 4-15, and Table 4-16, respectively. The Student-Newman Keuls test was applied to size and the results are show in Table 4-18 
and Table 4-19, and Table 4-20. The estimated marginal mean for the size and method factors are shown in Table 4-21, and Table 4-17.

Table 4-14 Software Test: Experiment 2, Contrast table for icon factor

\begin{tabular}{|c|c|c|}
\hline & & $\begin{array}{c}\text { Depende } \\
\text { nt } \\
\text { Variable }\end{array}$ \\
\hline Icon Difference Contr & & ErrorNOT \\
\hline Level 2 vs. Level 1 & Contrast Estimate & 67.231 \\
\hline & Hypothesized Value & 0 \\
\hline & Difference (Estimate - Hypothesized) & 67.231 \\
\hline & Std. Error & 1.817 \\
\hline & Sig. & .000 \\
\hline & 95\% Confidence Interval Lower Bound & 63.664 \\
\hline & for Difference Upper Bound & 70.799 \\
\hline Level 3 vs. Previous & Contrast Estimate & 64.104 \\
\hline & Hypothesized Value & 0 \\
\hline & Difference (Estimate - Hypothesized) & 64.104 \\
\hline & Std. Error & 1.574 \\
\hline & Sig. & .000 \\
\hline & $95 \%$ Confidence Interval Lower Bound & 61.014 \\
\hline & for Difference $\quad$ Upper Bound & 67.194 \\
\hline Level 4 vs. Previous & Contrast Estimate & 15.653 \\
\hline & Hypothesized Value & 0 \\
\hline & Difference (Estimate - Hypothesized) & 15.653 \\
\hline & Std. Error & 1.484 \\
\hline & Sig. & .000 \\
\hline & $95 \%$ Confidence Interval & 12.739 \\
\hline & for Difference $\quad$ Upper Bound & 18.566 \\
\hline Level 5 vs. Previous & Contrast Estimate & 41.887 \\
\hline & Hypothesized Value & 0 \\
\hline & Difference (Estimate - Hypothesized) & 41.887 \\
\hline & Std. Error & 1.436 \\
\hline & Sig. & .000 \\
\hline & $95 \%$ Confidence Interval Lower Bound & 39.067 \\
\hline & for Difference $\quad$ Upper Bound & 44.708 \\
\hline Level 6 vs. Previous & Contrast Estimate & -69.325 \\
\hline & Hypothesized Value & 0 \\
\hline & Difference (Estimate - Hypothesized) & -69.325 \\
\hline & Std. Error & 1.407 \\
\hline & Sig. & .000 \\
\hline & $95 \%$ Confidence Interval Lower Bound & -72.088 \\
\hline & Upper Bound & -66.561 \\
\hline
\end{tabular}


Table 4-15 Software Test: Experiment 2, Contrast table for size factor

\begin{tabular}{|c|c|c|c|}
\hline \multirow{2}{*}{\multicolumn{3}{|c|}{ Size Difference Contrast }} & $\begin{array}{c}\text { Depende } \\
\text { nt } \\
\text { Variable }\end{array}$ \\
\hline & & & ErrorNOT \\
\hline \multirow[t]{7}{*}{ Level 2 vs. Level 1} & \multicolumn{2}{|l|}{ Contrast Estimate } & 62.854 \\
\hline & \multicolumn{2}{|l|}{ Hypothesized Value } & 0 \\
\hline & \multicolumn{2}{|c|}{ Difference (Estimate - Hypothesized) } & 62.854 \\
\hline & \multicolumn{2}{|l|}{ Std. Error } & 1.285 \\
\hline & \multicolumn{2}{|l|}{ Sig. } & .000 \\
\hline & \multirow{2}{*}{$\begin{array}{l}95 \% \text { Confidence Interval } \\
\text { for Difference }\end{array}$} & Lower Bound & 60.331 \\
\hline & & Upper Bound & 65.376 \\
\hline \multirow[t]{7}{*}{ Level 3 vs. Previous } & \multirow{3}{*}{\multicolumn{2}{|c|}{$\begin{array}{l}\text { Contrast Estimate } \\
\text { Hypothesized Value } \\
\text { Difference (Estimate - Hypothesized) }\end{array}$}} & 160.308 \\
\hline & & & 0 \\
\hline & & & 160.308 \\
\hline & \multicolumn{2}{|l|}{ Std. Error } & 1.113 \\
\hline & \multicolumn{2}{|l|}{ Sig. } & .000 \\
\hline & \multirow{2}{*}{$\begin{array}{l}95 \% \text { Confidence Interval } \\
\text { for Difference }\end{array}$} & Lower Bound & 158.123 \\
\hline & & Upper Bound & 162.493 \\
\hline
\end{tabular}

Table 4-16 Software Test: Experiment 2, Contrast table for method factor

\begin{tabular}{|ll|r|}
\hline Method Difference & & $\begin{array}{c}\text { Depende } \\
\text { nt } \\
\text { Variable }\end{array}$ \\
\cline { 3 - 3 } Contrast & ErrorNOT \\
\hline Level 2 vs. Level 1 & Contrast Estimate & 86.513 \\
& Hypothesized Value & 0 \\
& Difference (Estimate - Hypothesized) & 86.513 \\
& Std. Error & 1.049 \\
& Sig. & .000 \\
95\% Confidence Interval $\quad$ Lower Bound & 84.453 \\
for Difference & Upper Bound & 88.573 \\
\hline
\end{tabular}

Table 4-17 Software Test: Experiment 2, Estimated marginal means for method factor

\begin{tabular}{|l|l|r|r|r|}
\hline & & & \multicolumn{2}{|c|}{$95 \%$ Confidence Interval } \\
\cline { 4 - 5 } Method & Mean & Std. Error & Lower Bound & Upper Bound \\
\hline 1.00 & 193.996 & .742 & 192.539 & 195.452 \\
2.00 & 280.508 & .742 & 279.052 & 281.965 \\
\hline
\end{tabular}


Table 4-18 Software Test: Experiment 2, S-N-K table for icon factor

\begin{tabular}{|l|r|r|r|r|}
\hline & & \multicolumn{3}{|c|}{ Subset } \\
\cline { 3 - 5 } icon & $\mathrm{N}$ & 1 & \multicolumn{1}{|c|}{2} & \multicolumn{1}{|c|}{3} \\
\hline 6.00 & 120 & 179.4814 & & \\
1.00 & 120 & 181.5318 & & \\
2.00 & 120 & & 248.7633 & \\
4.00 & 120 & & 252.1680 & \\
3.00 & 120 & & & 279.2514 \\
5.00 & 120 & & & 282.3157 \\
Sig. & & .260 & .061 & .092 \\
\hline
\end{tabular}

Table 4-19 Software Test: Experiment 2, S-N-K for size factor

\begin{tabular}{|l|r|r|r|r|}
\hline & & \multicolumn{3}{|c|}{ Subset } \\
\cline { 3 - 5 } Size & \multicolumn{1}{|c|}{$\mathrm{N}$} & \multicolumn{1}{|c|}{1} & \multicolumn{1}{c|}{3} \\
\hline 1.00 & 240 & 152.3892 & & \\
2.00 & 240 & & 215.2428 & \\
3.00 & 240 & & & 344.1239 \\
Sig. & & 1.000 & 1.000 & 1.000 \\
\hline
\end{tabular}

Table 4-20 Software Test: Experiment 2, Estimated marginal means for icon factor

\begin{tabular}{|l|l|r|r|r|}
\hline & & & \multicolumn{2}{|c|}{$95 \%$ Confidence Interval } \\
\cline { 4 - 5 } Icon & Mean & Std. Error & Lower Bound & Upper Bound \\
\hline 1.00 & 181.532 & 1.285 & 179.009 & 184.055 \\
2.00 & 248.763 & 1.285 & 246.240 & 251.286 \\
3.00 & 279.251 & 1.285 & 276.729 & 281.774 \\
4.00 & 252.168 & 1.285 & 249.645 & 254.691 \\
5.00 & 282.316 & 1.285 & 279.793 & 284.839 \\
6.00 & 179.481 & 1.285 & 176.959 & 182.004 \\
\hline
\end{tabular}

Table 4-21 Software Test: Experiment 2, Estimated marginal means for size factor

\begin{tabular}{|l|l|r|r|r|}
\hline & & & \multicolumn{2}{|c|}{$95 \%$ Confidence Interval } \\
\cline { 4 - 5 } Size & Mean & Std. Error & Lower Bound & Upper Bound \\
\hline 1.00 & 152.389 & .908 & 150.605 & 154.173 \\
2.00 & 215.243 & .908 & 213.459 & 217.027 \\
3.00 & 344.124 & .908 & 342.340 & 345.908 \\
\hline
\end{tabular}




\section{$\underline{\text { Key Findings }}$}

All of the factors of interest tested in the ANOVA were found to be significant, including all of the interactions $(\mathrm{p}<0.005)$. The method factor resulted in a $54 \%$ decrease in the untransformed similarity factor when comparing blurred retinal images to precompensated retinal images (1536.2 vs. 823.03 , respectively). The size*method interaction was found to be significant indicating that perhaps the method will work better for certain sizes when testing repeated measures. Method one resulted in a lower but different Difference Measure across all icons pointing to the possibility that the precompensation process may perform better for certain icon designs, than for others.

\subsection{Artificial Eye Test Results}

The images captured through the artificial eye for normal and precompensated images are shown, as well as the PSFs for each lens used in the inverse process and the original icon. These images were generated with $\mathrm{k}=0.005$ in the deconvolution step, Equation 3-6. After the precompensated images were generated, they were postprocessed using Contrast-Limited Adaptive Histogram Equalization using the MATLAB ${ }^{\circledR}$ function, adapthisteq with the default parameters.

As can be seen from Figure 4-6, the retinal image captures through the artificial are more clearly defined for the precompensated images as compared to the normal images, although the background gray level appears changed and contrast has been reduced.

The data for the artificial eye experiment was collected as a repeated measures experiment but is treated as a Randomized Block Design, consisting of the subject factor 
(random: five levels), the Icon factor ( fixed: six levels), the Size factor (fixed: four levels), and the Method factor (fixed: two levels). This is done because a repeated measure design is a special case of randomized block design with blocking on the subject factor [33]. Treating the experiment in this way allows for easy assumption verification in SPSS $^{\circledR}$. Thus, a $240 \times 5$ matrix of data was used in $\operatorname{SPSS}^{\circledR}$ to perform the statistical analysis. The data was first screened for outliers, normality and homogeneity of variance. The first pass of the data screening revealed that the data was not normal. A square root transformation was applied to the data and then the transformed data was screened. The results are as follows.

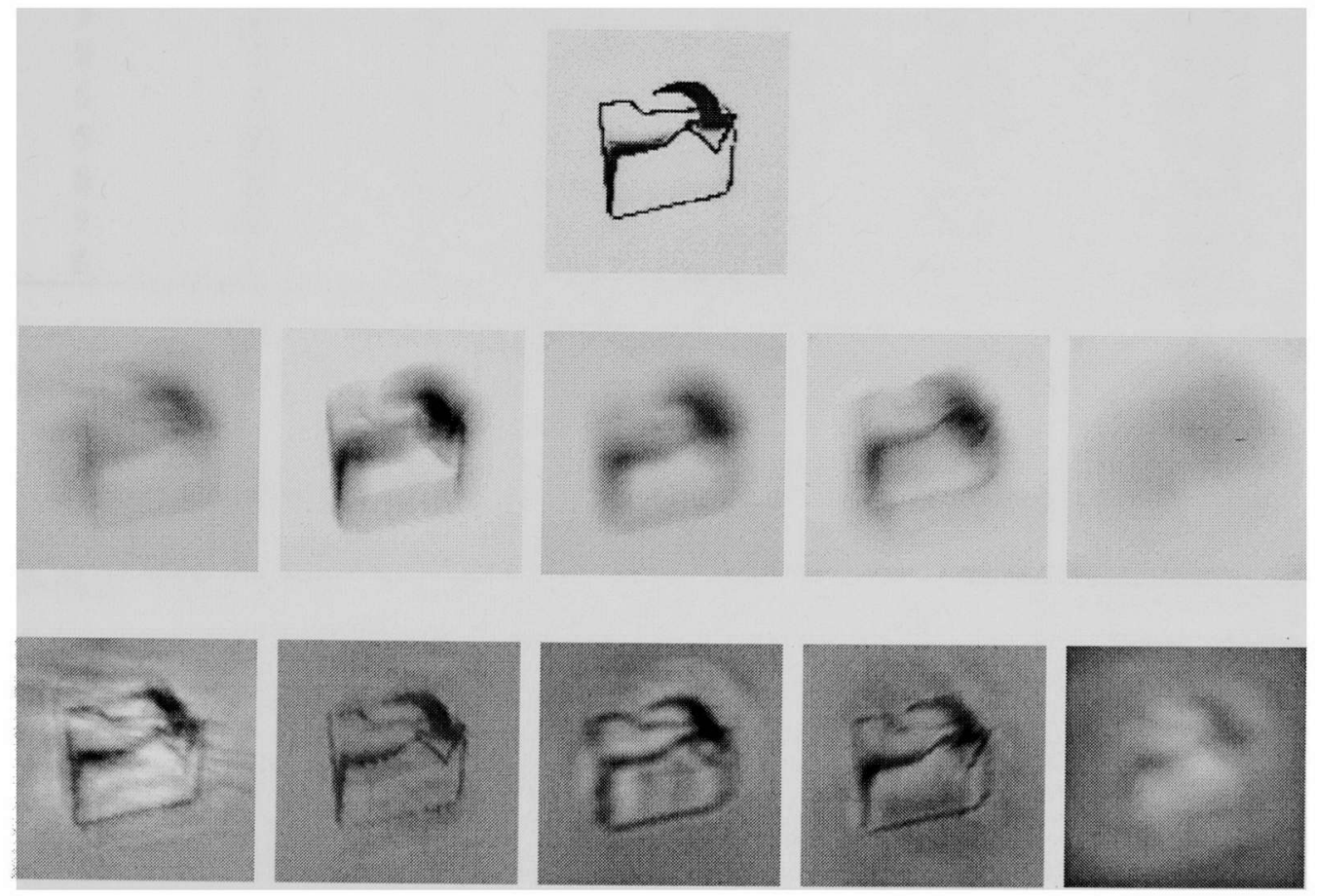

Figure 4-6 Folder icon with retinal image capture of normal and precompensated versions for each artificial eye lens. Top - original icon capture through unaberrated artificial eye. First row - uncompensated icon through each lens. Bottom row - Corresponding precompensated icon capture. 
Outliers were assessed by analyzing the distribution of the Studentized residuals. The Studentized residuals were plotted against a $t_{59}$ distribution. A $95 \%$ confidence interval was used as a cutoff for detecting outliers. Nine outliers were detected in the data and they were replaced with the fitted values. These outliers represent $3.75 \%$ of the data. This is shown in Table 4-22.

Table 4-22 Artificial Eye Test: Outliers

\begin{tabular}{ccccccc} 
Lens & Icon & Size & Method & Error & Sqrt of Error & Replaced Values \\
\hline 1 & 1 & 4 & 2 & 4069.05 & 63.79 & 72.31 \\
2 & 4 & 1 & 2 & 3308.73 & 57.52 & 64.51 \\
5 & 2 & 4 & 2 & 5741.89 & 75.78 & 75.78 \\
5 & 1 & 3 & 2 & 4948.15 & 70.34 & 63.05 \\
5 & 3 & 3 & 2 & 4917.96 & 70.13 & 62.17 \\
5 & 5 & 1 & 2 & 5477.14 & 74.01 & 60.34 \\
5 & 5 & 3 & 2 & 6313.2 & 79.46 & 65.61 \\
5 & 4 & 1 & 2 & 7966.97 & 89.26 & 74.86 \\
5 & 1 & 4 & 2 & 8771.57 & 93.66 & 79.22 \\
\hline
\end{tabular}

\section{Normality}

Normality was assessed by testing the Studentized residuals against a Tdistribution. One of the assumptions of the ANOVA is that the distribution of the residuals is normal. A T-quantile plot $(\mathrm{DF}=59)$, a histogram, and both skewness and kurtosis values were obtained for the data. The residuals of the data are approximately normal for the histogram and quantile plots. The skewness and kurtosis values do not indicate any strong deviation from normality. Figure 4-7, Figure 4-8, and Table 4-23 summarize these results. 


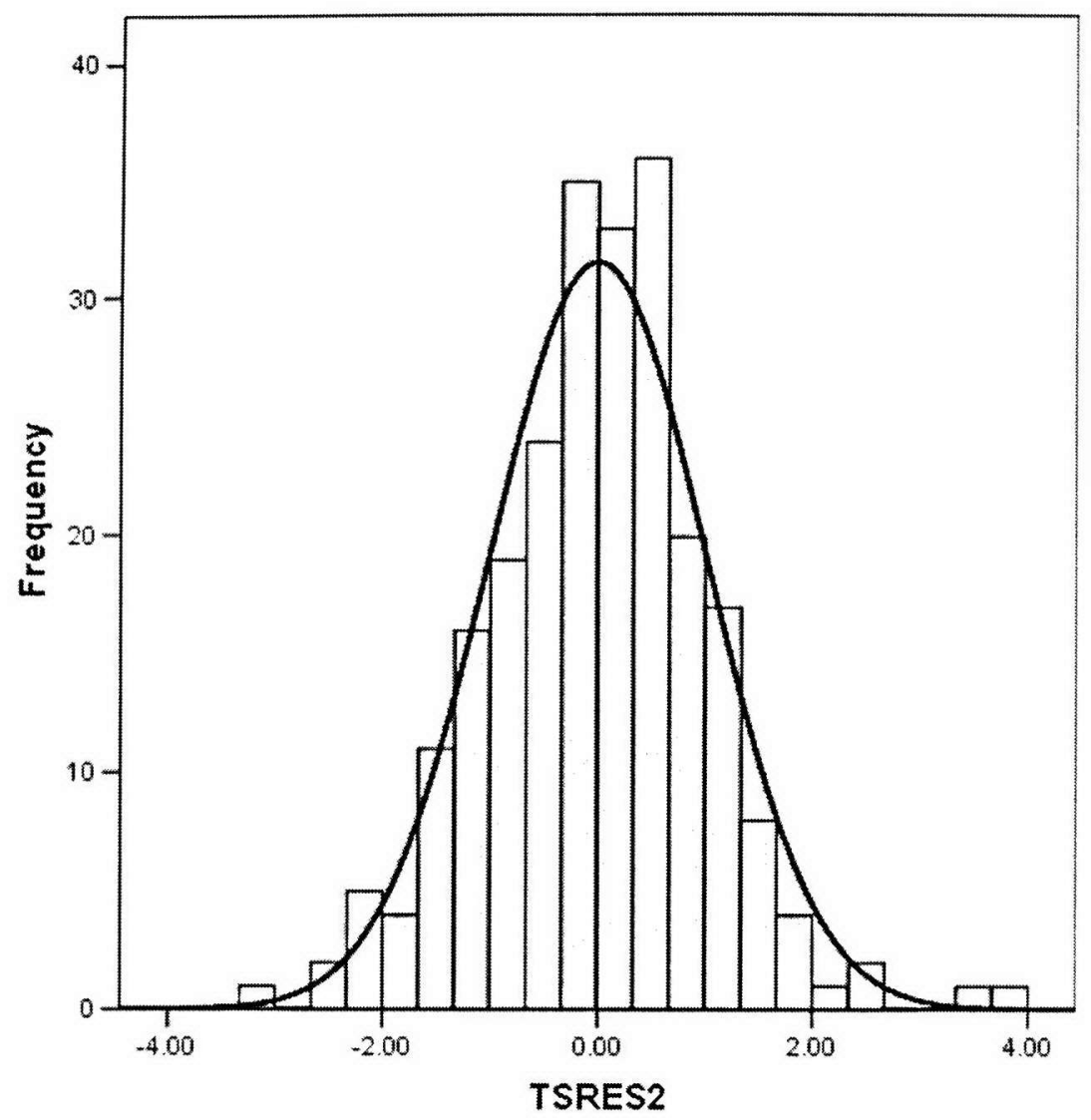

Figure 4-7 Artificial Eye Test: Histogram of the residuals

Homogeneity of Variance

The homogeneity of variance was tested by applying Levene's test and the assumption of equal variances was met. The results of the Levene test are shown in Table $4-24$.

$\underline{\text { ANOVA }}$

Upon verification of the required assumptions, an ANOVA was applied to the data. The results obtained are shown in Table 4-25. The results of the contrasts on icon, size, and method are shown in Table 4-26, Table 4-27, and Table 4-28, respectively. The Student- 
Newman Keuls test was applied to size and the results are show in Table 4-29 and Table 4-30. The estimated marginal means for the size and method factors are shown in Table 4-31, Table 4-32, and Table 4-33.

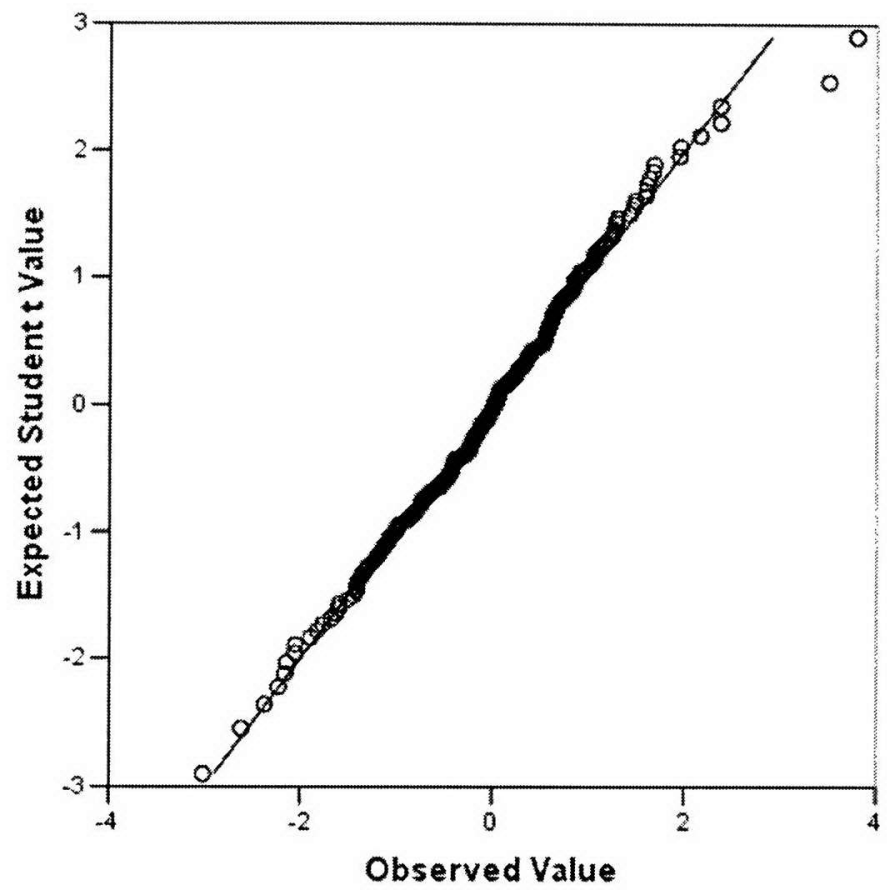

Figure 4-8 Artificial Eye Test: T-quantile plot of the Studentized residuals

Table 4-23 Artificial Eye Test: Skewness and Kurtosis for the Studentized residuals

\begin{tabular}{|l|r|r|r|r|r|}
\hline & \multicolumn{1}{|c|}{$\mathrm{N}$} & \multicolumn{2}{|c|}{ Skewness } & \multicolumn{2}{c|}{ Kurtosis } \\
\cline { 2 - 6 } & \multicolumn{1}{|c|}{ Statistic } & Statistic & \multicolumn{1}{c|}{ Std. Error } & Statistic & Std. Error \\
\hline TSRES2 & 240 & .111 & .157 & .984 & .313 \\
Valid N (listwise) & 240 & & & & \\
\hline
\end{tabular}


Table 4-24 Artificial Eye Test: Levene's test for homogeneity of variance

\begin{tabular}{|ll|r|r|r|r|}
\hline & $\begin{array}{r}\text { Levene } \\
\text { Statistic }\end{array}$ & \multicolumn{1}{c|}{ df1 } & \multicolumn{1}{c|}{ df2 } & \multicolumn{1}{c|}{ Sig. } \\
\hline ET & .756 & 47 & 192 & .871 \\
& Based on Mean & .439 & 47 & 192 & .999 \\
Based on Median & .439 & 47 & 114.295 & .999 \\
Based on Median and & .699 & 47 & 192 & .927 \\
with adjusted df & & & \\
Based on trimmed mean & & &
\end{tabular}

Table 4-25 Artificial Eye Test: ANOVA table

\begin{tabular}{|ll|r|r|r|r|r|}
\hline Source & & $\begin{array}{c}\text { Type III Sum } \\
\text { of Squares }\end{array}$ & df & Mean Square & \multicolumn{1}{c|}{ F } & Sig. \\
\hline Intercept & Hypothesis & 301104.903 & 1 & 301104.903 & 655.310 & .000 \\
Icon & Error & 1837.939 & 4 & $459.485^{\mathrm{a}}$ & & \\
& Hypothesis & 1150.719 & 5 & 230.144 & 60.430 & .000 \\
Size & Error & 715.989 & 188 & $3.808^{\mathrm{b}}$ & & \\
& Hypothesis & 5475.548 & 3 & 1825.183 & 479.245 & .000 \\
Method & Error & 715.989 & 188 & $3.808^{\mathrm{b}}$ & & \\
& Hypothesis & 13227.981 & 1 & 13227.981 & 3473.320 & .000 \\
Lens & Error & 715.989 & 188 & $3.808^{\mathrm{b}}$ & & \\
& Hypothesis & 1837.939 & 4 & 459.485 & 120.649 & .000 \\
Icon * Size & Error & 715.989 & 188 & $3.808^{\mathrm{b}}$ & & \\
& Hypothesis & 27368.073 & 15 & 1824.538 & 479.076 & .000 \\
Icon * Method & Error & 715.989 & 188 & $3.808^{\mathrm{b}}$ & & \\
& Hypothesis & 142.263 & 5 & 28.453 & 7.471 & .000 \\
Size * Method & Error & 715.989 & 188 & $3.808^{\mathrm{b}}$ & & \\
& Hypothesis & 190.125 & 3 & 63.375 & 16.641 & .000 \\
Icon * Size * & Error & 715.989 & 188 & $3.808^{\mathrm{b}}$ & & \\
Method & Hypothesis & 1459.866 & 15 & 97.324 & 25.555 & .000 \\
\hline
\end{tabular}


Table 4-26 Artificial Eye Test: Contrast table for icon factor

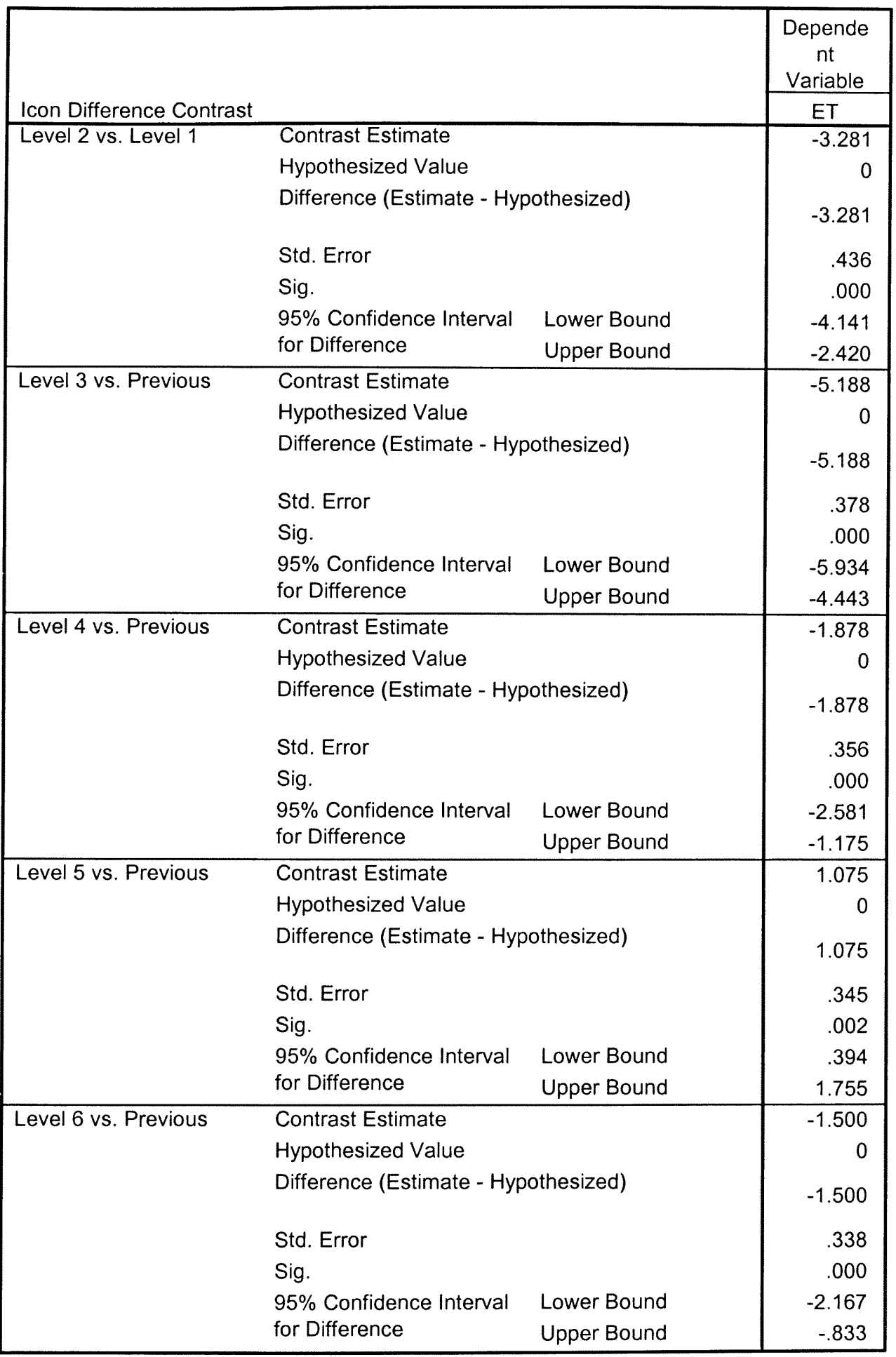


Table 4-27 Artificial Eye Test: Contrast table for size factor

\begin{tabular}{|c|c|c|c|}
\hline & & & $\begin{array}{c}\text { Depende } \\
\text { nt } \\
\text { Variable } \\
\end{array}$ \\
\hline Size Difference Cont & & & ET \\
\hline Level 2 vs. Level 1 & Contrast Estimate & & .540 \\
\hline & Hypothesized Value & & 0 \\
\hline & Difference (Estimate - Hyp & thesized) & .540 \\
\hline & Std. Error & & .356 \\
\hline & Sig. & & .131 \\
\hline & $95 \%$ Confidence Interval & Lower Bound & -.163 \\
\hline & for Difference & Upper Bound & 1.243 \\
\hline Level 3 vs. Previous & Contrast Estimate & & 11.688 \\
\hline & Hypothesized Value & & 0 \\
\hline & Difference (Estimate - Hyp & thesized) & 11.688 \\
\hline & Std. Error & & .309 \\
\hline & Sig. & & .000 \\
\hline & $95 \%$ Confidence Interval & Lower Bound & 11.080 \\
\hline & for Difference & Upper Bound & 12.297 \\
\hline Level 4 vs. Previous & Contrast Estimate & & .222 \\
\hline & Hypothesized Value & & 0 \\
\hline & Difference (Estimate - Hyp & thesized) & .222 \\
\hline & Std. Error & & .291 \\
\hline & Sig. & & .447 \\
\hline & $95 \%$ Confidence Interval & Lower Bound & -.352 \\
\hline & for Difference & Upper Bound & .796 \\
\hline
\end{tabular}

Table 4-28 Artificial Eye Test: Contrast table for method factor

\begin{tabular}{|ll|r|}
\hline Method Difference & & $\begin{array}{c}\text { Depende } \\
\text { nt } \\
\text { Variable }\end{array}$ \\
\cline { 3 - 3 } Contrast & \multicolumn{1}{|c|}{ ET } \\
\hline Level 2 vs. Level 1 & Contrast Estimate & 14.848 \\
& Hypothesized Value & 0 \\
& Difference (Estimate - Hypothesized) & 14.848 \\
& Std. Error & .252 \\
& Sig. & .000 \\
& $95 \%$ Confidence Interval $\quad$ Lower Bound & 14.351 \\
& for Difference & 15.345 \\
\hline
\end{tabular}


Table 4-29 Artificial Eye Test: S-N-K for icon factor

\begin{tabular}{|l|r|r|r|r|r|}
\hline & \multirow{2}{*}{ Icon } & $\mathrm{N}$ & \multicolumn{4}{|c|}{ Subset } \\
\cline { 3 - 6 } & \multicolumn{1}{|c|}{1} & 2 & \multicolumn{1}{|c|}{3} \\
\hline 3.00 & 40 & 32.4662 & & & \\
4.00 & 40 & & 34.0471 & & \\
6.00 & 40 & & 34.1705 & & \\
2.00 & 40 & & & 36.0140 & \\
5.00 & 40 & & & 36.5300 & \\
1.00 & 40 & & & & 39.2945 \\
Sig. & & 1.000 & .778 & .238 & 1.000 \\
\hline
\end{tabular}

Table 4-30 Artificial Eye Test: S-N-K for size factor

\begin{tabular}{|l|r|r|r|r|}
\hline & \multirow{2}{*}{ Size } & $N$ & \multicolumn{3}{|c|}{ Subset } \\
\cline { 3 - 5 } & $\mathrm{N}$ & \multicolumn{1}{|c|}{2} & \multicolumn{1}{c|}{3} \\
\hline 1.00 & 60 & 31.1988 & & \\
2.00 & 60 & 31.7389 & & \\
4.00 & 60 & & 35.5868 & \\
3.00 & 60 & & & 43.1571 \\
Sig. & & .131 & 1.000 & 1.000 \\
\hline
\end{tabular}

Table 4-31 Artificial Eye Test: Estimated marginal means for icon factor

\begin{tabular}{|l|c|r|r|r|}
\hline & & & \multicolumn{2}{|c|}{$95 \%$ Confidence Interval } \\
\cline { 4 - 5 } Icon & Mean & Std. Error & Lower Bound & Upper Bound \\
\hline 1.00 & 39.295 & .309 & 38.686 & 39.903 \\
2.00 & 36.014 & .309 & 35.405 & 36.623 \\
3.00 & 32.466 & .309 & 31.857 & 33.075 \\
4.00 & 34.047 & .309 & 33.438 & 34.656 \\
5.00 & 36.530 & .309 & 35.921 & 37.139 \\
6.00 & 34.171 & .309 & 33.562 & 34.779 \\
\hline
\end{tabular}

Table 4-32 Artificial Eye Test: Estimated marginal means for size factor

\begin{tabular}{|l|c|r|r|r|}
\hline & & & \multicolumn{2}{|c|}{$95 \%$ Confidence Interval } \\
\cline { 4 - 5 } Size & Mean & Std. Error & Lower Bound & Upper Bound \\
\hline 1.00 & 31.199 & .252 & 30.702 & 31.696 \\
2.00 & 31.739 & .252 & 31.242 & 32.236 \\
3.00 & 43.157 & .252 & 42.660 & 43.654 \\
4.00 & 35.587 & .252 & 35.090 & 36.084 \\
\hline
\end{tabular}




\begin{tabular}{|l|c|r|r|r|}
\hline & & & \multicolumn{2}{|c|}{$95 \%$ Confidence Interval } \\
\cline { 4 - 5 } Method & Mean & Std. Error & Lower Bound & Upper Bound \\
\hline 1.00 & 27.996 & .178 & 27.645 & 28.348 \\
2.00 & 42.844 & .178 & 42.493 & 43.196 \\
\hline
\end{tabular}

\section{$\underline{\text { Key Findings }}$}

All the main effects and interactions were significant $(\mathrm{p}<0.005)$, suggesting that the precompensation algorithm can successfully improve the similarity of the icons displayed for the camera system, with means of 783.8 vs. 1835.6 for method one and two for the Difference Measure, respectively. The interactions found may be indicative that each unique lens may produce Difference Measures that cause significant variation from the mean, depending on the icon-size combination.

\subsection{Human Subject Test Results}

\subsubsection{Experiment 1}

Experiment one for the experimentation with human subjects is a factorial experiment blocking on the icon factor. The data collected for this experiment consists of five columns of data, each representing a treatment combination on twenty unique subjects. SPSS ${ }^{\circledR}$ was used to perform the statistical analysis. The data was first screened for outliers, normality and homogeneity of variance.

\section{$\underline{\text { Outliers }}$}

Outliers were assessed by analyzing the distribution of the Studentized residuals. The Studentized residuals were plotted against a $t_{56}$ distribution. A 95\% confidence interval was used as a cutoff for detecting outliers. Six outliers were detected in the data 
and they were replaced with the fitted values. These outliers represent $7.5 \%$ of the data. This is shown in Table 4-34.

Table 4-34 Human Subject Test: Experiment 1, Outliers

\begin{tabular}{cccccc} 
Group & Eye & Method & Subject & Acuity & Replaced Value \\
\hline 3 & 2 & 1 & 11 & 0 & 0.23 \\
3 & 1 & 2 & 12 & 0.04 & 0.26 \\
3 & 1 & 2 & 11 & 0.5 & 0.17 \\
3 & 2 & 1 & 12 & 0.62 & 0.33 \\
4 & 2 & 2 & 19 & 0.44 & 0.22 \\
4 & 2 & 1 & 17 & 0.3 & 0.09 \\
\hline
\end{tabular}

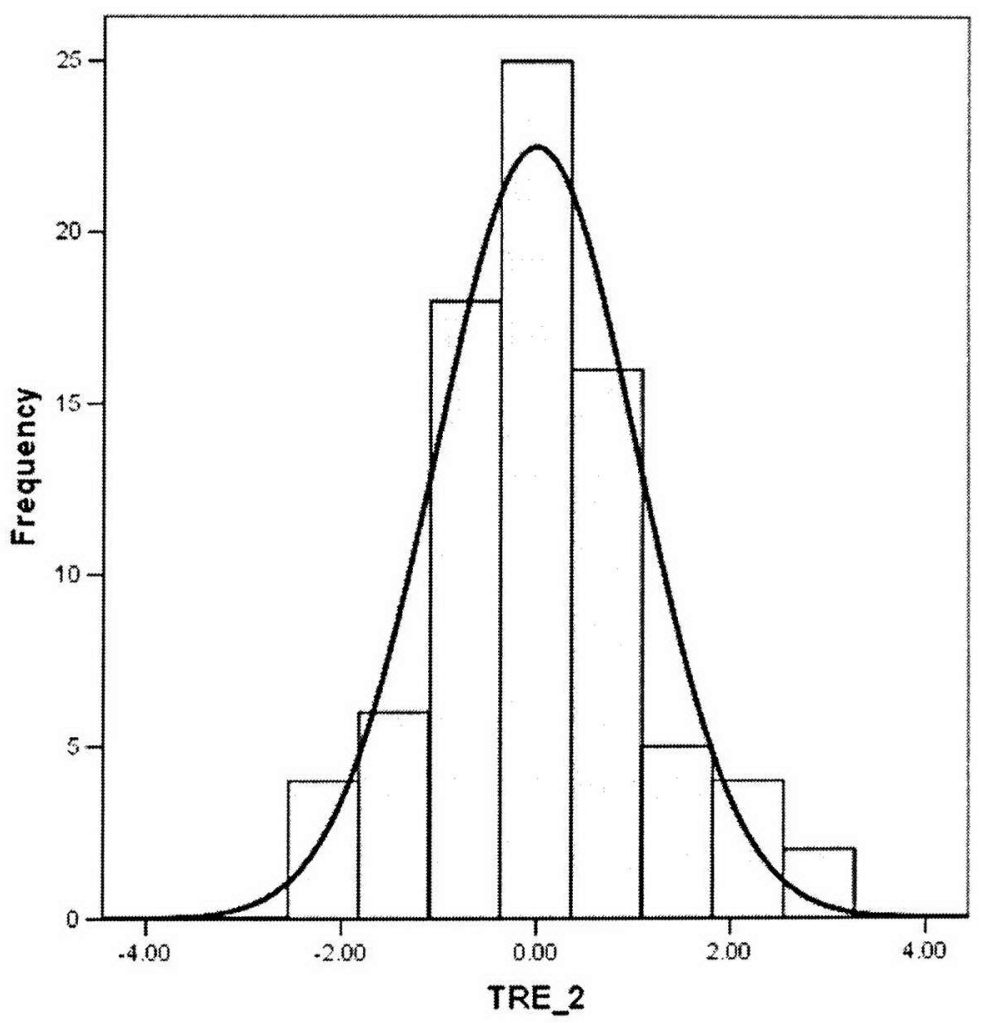

Figure 4-9 Human Subject Test: Experiment 1, Histogram of residuals 


\section{Normality}

Normality was assessed by testing the normality of the Studentized residuals. One of the assumptions of the ANOVA is that the distribution of the residuals is normal. A Tquantile plot $(\mathrm{DF}=56)$, a histogram, and both skewness and kurtosis values were obtained for the data. The residuals of the data are approximately normal for the histogram and quantile plots. The skewness and kurtosis values do not indicate any strong deviation from normality. Figure 4-9, Figure 4-10, and Table 4-35 summarize these results.

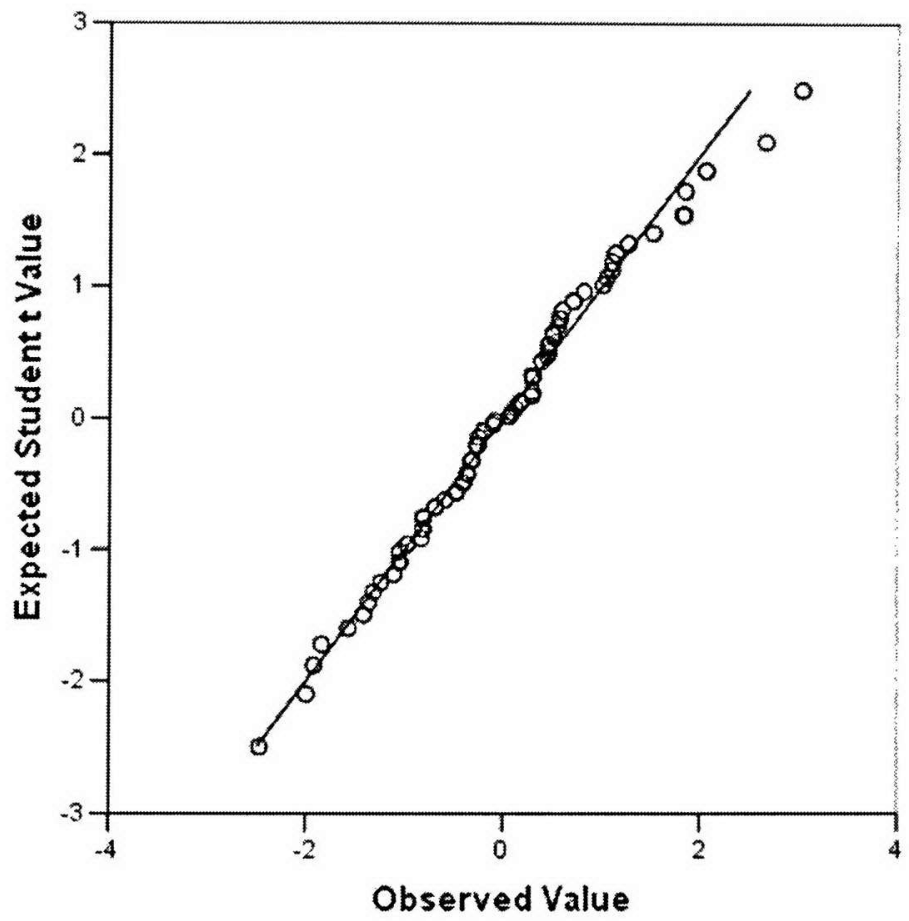

Figure 4-10 Human Subject Test: Experiment 1, T-quantile plot of residuals

Table 4-35 Human Subject Test: Experiment 1, Skewness and Kurtosis values for residuals

\begin{tabular}{|l|r|r|r|r|r|}
\hline & \multicolumn{1}{|c|}{$\mathrm{N}$} & \multicolumn{2}{|c|}{ Skewness } & \multicolumn{2}{c|}{ Kurtosis } \\
\cline { 2 - 6 } & Statistic & \multicolumn{1}{|c|}{ Statistic } & Std. Error & \multicolumn{1}{c|}{ Statistic } & Std. Error \\
\hline TRE_2 & 80 & .326 & .269 & .575 & .532 \\
Valid N (listwise) & 80 & & & & \\
\hline
\end{tabular}




\section{Homogeneity of Variance}

The homogeneity of variance was tested by applying Levene's statistic to the distribution of each cell of the model. The assumption of equal variances was not met and no transformation could be found that would make the variances equal, but this is still acceptable, as mentioned in the previous section. The results of the Levene test are shown in Table 4-36.

Table 4-36 Human Subject Test: Experiment 1, Levene Test for Homogeneity of Variance

\begin{tabular}{|ll|r|r|r|r|}
\hline & Levene & \multicolumn{1}{|c|}{ df1 } & \multicolumn{1}{c|}{ df2 } & \multicolumn{1}{c|}{ Sig. } \\
\hline AcuityNO & Based on Mean & 4.450 & 11 & 48 & .000 \\
& Based on Median & 1.090 & 11 & 48 & .389 \\
& Based on Median and & 1.090 & 11 & 23.453 & .410 \\
& with adjusted df & 4.215 & 11 & 48 & .000 \\
\hline
\end{tabular}

\section{$\underline{\text { ANOVA }}$}

Upon verification of the required assumptions, an ANOVA was applied to the data. The results obtained are shown in Table 4-37. Unfortunately, SPSS ${ }^{\circledR}$ does not allow for contrasts using the mixed linear model analysis. Main effects comparisons, however, were provided with the analysis. The estimated means and pair-wise comparisons of groups are shown in Table 4-39 and Table 4-41. The estimated marginal means for the significant interactions are shown in Table 4-42 and Table 4-40. 
Table 4-37 Human Subject Tests: Experiment 1, ANOVA of Fixed Effects

\begin{tabular}{|l|r|r|r|r|}
\hline Source & Numerator df & $\begin{array}{r}\text { Denominator } \\
\text { df }\end{array}$ & \multicolumn{1}{|c|}{ F } & \multicolumn{1}{c|}{ Sig. } \\
\hline$G$ & 3 & 14.974 & 3.304 & .049 \\
$E$ & 1 & 32.706 & .092 & .764 \\
$M$ & 1 & 32.706 & .477 & .494 \\
$G{ }^{*} E$ & 3 & 32.706 & 3.036 & .043 \\
$G{ }^{*} M$ & 3 & 32.706 & 1.996 & .134 \\
$E^{*} M$ & 1 & 32.706 & 4.845 & .035 \\
$G{ }^{*} E * M$ & 3 & 32.706 & 2.653 & .065 \\
\hline
\end{tabular}

Table 4-38 Human Subject Tests: Experiment 1, Covariates

\begin{tabular}{|ll|r|r|}
\hline Parameter & & Estimate & Std. Error \\
\hline Repeated & Var: $[E=1.00]^{*}[M=1.00]$ & .004647 & .002645 \\
Measures & Var: $[E=1.00]^{*}[M=2.00]$ & .007618 & .003909 \\
& Var: $[E=2.00]^{*}[M=1.00]$ & .002440 & .002171 \\
& Var: $[E=2.00]^{*}[M=2.00]$ & .019325 & .007288 \\
$P(G)$ [subject = P] & Variance & .016335 & .006793 \\
\hline
\end{tabular}

Table 4-39 Human Subject Tests: Experiment 1, Estimated Means for Groups

\begin{tabular}{|l|r|r|r|r|r|}
\hline & & & & \multicolumn{2}{|c|}{$95 \%$ Confidence Interval } \\
\cline { 5 - 6 } $\mathrm{G}$ & Mean & Std. Error & $\mathrm{df}$ & Lower Bound & Upper Bound \\
\hline 1.00 & .021 & .061 & 14.974 & -.109 & .151 \\
2.00 & .264 & .061 & 14.974 & .134 & .394 \\
3.00 & .124 & .061 & 14.974 & -.006 & .253 \\
4.00 & .041 & .061 & 14.974 & -.089 & .170 \\
\hline
\end{tabular}

Table 4-40 Human Subject Test: Experiment 1, E*M Interaction Means

\begin{tabular}{|ll|r|r|r|r|r|}
\hline & & & & & \multicolumn{2}{|c|}{$95 \%$ Confidence Interval } \\
\cline { 6 - 7 } & $M$ & Mean & Std. Error & df & Lower Bound & Upper Bound \\
\hline 1.00 & 1.00 & .139 & .032 & 21.240 & .072 & .206 \\
& 2.00 & .079 & .035 & 24.832 & .008 & .151 \\
\hline 2.00 & 1.00 & .100 & .031 & 11.432 & .033 & .167 \\
& 2.00 & .131 & .042 & 25.202 & .044 & .218 \\
\hline
\end{tabular}


Table 4-41 Human Subject Tests: Experiment 1, Pair-wise Comparison of Groups

\begin{tabular}{|c|c|c|c|c|c|c|c|}
\hline \multirow[b]{2}{*}{ (I) $G$} & \multirow[b]{2}{*}{ (J) $G$} & \multirow{2}{*}{$\begin{array}{c}\text { Mean } \\
\text { Difference } \\
(1-J)\end{array}$} & \multirow[b]{2}{*}{ Std. Error } & \multirow[b]{2}{*}{$\mathrm{df}$} & \multirow[b]{2}{*}{ Sig. $^{a}$} & \multicolumn{2}{|c|}{$\begin{array}{l}\text { 95\% Confidence Interval for } \\
\text { Difference }^{\mathrm{a}}\end{array}$} \\
\hline & & & & & & Lower Bound & Upper Bound \\
\hline \multirow[t]{3}{*}{1.00} & 2.00 & $-.243^{\star}$ & .086 & 14.974 & .013 & -.426 & -.060 \\
\hline & 3.00 & -.103 & .086 & 14.974 & .252 & -.286 & .081 \\
\hline & 4.00 & -.020 & .086 & 14.974 & .822 & -.203 & .163 \\
\hline \multirow[t]{3}{*}{2.00} & 1.00 & $.243^{\star}$ & .086 & 14.974 & .013 & .060 & .426 \\
\hline & 3.00 & .141 & .086 & 14.974 & .123 & -.043 & .324 \\
\hline & 4.00 & $.223^{*}$ & .086 & 14.974 & .020 & .040 & .406 \\
\hline \multirow[t]{3}{*}{3.00} & 1.00 & .103 & .086 & 14.974 & .252 & -.081 & .286 \\
\hline & 2.00 & -.141 & .086 & 14.974 & .123 & -.324 & .043 \\
\hline & 4.00 & .083 & .086 & 14.974 & .351 & -.100 & .266 \\
\hline \multirow[t]{3}{*}{4.00} & 1.00 & .020 & .086 & 14.974 & .822 & -.163 & .203 \\
\hline & 2.00 & $-.223^{\star}$ & .086 & 14.974 & .020 & -.406 & -.040 \\
\hline & 3.00 & -.083 & .086 & 14.974 & .351 & -.266 & .100 \\
\hline
\end{tabular}

Table 4-42 Human Subject Test: Experiment 1, E*G Interaction Means

\begin{tabular}{|ll|r|r|r|r|r|}
\hline & & & & & \multicolumn{2}{|c|}{$95 \%$ Confidence Interval } \\
\cline { 5 - 7 } & $\mathrm{G}$ & \multicolumn{1}{|c|}{ Mean } & Std. Error & \multicolumn{1}{c|}{ df } & Lower Bound & Upper Bound \\
\hline 1.00 & 1.00 & $-6.9 \mathrm{E}-018$ & .062 & 18.200 & -.131 & .131 \\
& 2.00 & .306 & .062 & 18.200 & .175 & .437 \\
& 3.00 & .131 & .062 & 18.200 & $-6.61 \mathrm{E}-005$ & .261 \\
& 4.00 & $-5.6 \mathrm{E}-017$ & .062 & 18.200 & -.131 & .131 \\
\hline 2.00 & 1.00 & .042 & .066 & 17.942 & -.097 & .181 \\
& 2.00 & .222 & .066 & 17.942 & .083 & .361 \\
& 3.00 & .116 & .066 & 17.942 & -.022 & .255 \\
4.00 & .081 & .066 & 17.942 & -.057 & .220 \\
\hline
\end{tabular}

\section{$\underline{\text { Key Findings }}$}

The Group factor, the Group*Eye, and the Eye*Method Interactions were found to be significant at the $5 \%$ significance level $(\mathrm{p}=0.049, \mathrm{p}=0.043$, and $\mathrm{p}=0.035$, respectively). The method factor, the primary factor of interest, is not significant. This may be evidence the acuity test did not challenge the subjects enough to assess their true acuity. Although there was significance among groups, it is not a very strong 
significance, noting that it is only slightly less than the $5 \%$ significance level. These findings will be discussed in further detail in chapter five.

\subsubsection{Experiment 2}

Experiment two for the experimentation with human subjects is a factorial experiment blocking on the icon factor. The data collected for this experiment consists of 6 columns of data, each representing a treatment combination on 20 unique subjects. SPSS $^{\circledR}$ was used to perform the statistical analysis. The data was first screened for outliers, normality and homogeneity of variance. Initially, the data was slightly nonnormal. After applying a power transform, with $b=4$, the data was found to be normal.

\section{$\underline{\text { Outliers }}$}

Outliers were assessed by analyzing the distribution of the Studentized residuals. The Studentized residuals were plotted against a $t_{113}$ distribution. A $95 \%$ confidence interval was used as a cutoff for detecting outliers.

Table 4-43 Human Subject Test: Experiment 2, Outliers

\begin{tabular}{|c|c|c|c|c|c|c|}
\hline Group & Size & Eye & Method & Subject & Correct & Replaced Value \\
\hline 2 & 3 & 1 & 1 & 11 & 2 & 7.52 \\
\hline 4 & 1 & 1 & 1 & 20 & 5 & 10.33 \\
\hline 3 & 1 & 2 & 1 & 1 & 3 & 8.02 \\
\hline 3 & 1 & 1 & 2 & 6 & 1 & 5.57 \\
\hline 3 & 2 & 2 & 1 & 6 & 2 & 5.37 \\
\hline 2 & 1 & 1 & 2 & 4 & 1 & 4.35 \\
\hline 3 & 1 & 1 & 1 & 1 & 5 & 8.22 \\
\hline 2 & 3 & 2 & 2 & 4 & 2 & 5.15 \\
\hline 2 & 2 & 1 & 2 & 4 & 12 & 6.75 \\
\hline 2 & 3 & 1 & 1 & 4 & 9 & 4.35 \\
\hline 3 & 2 & 1 & 1 & 1 & 12 & 7.82 \\
\hline 2 & 3 & 2 & 2 & 11 & 12 & 8.32 \\
\hline 2 & 1 & 1 & 2 & 11 & 11 & 7.52 \\
\hline 3 & 2 & 2 & 2 & 6 & 11 & 7.57 \\
\hline 3 & 1 & 2 & 1 & 6 & 7 & 3.77 \\
\hline 3 & 2 & 1 & 2 & 6 & 10 & 6.77 \\
\hline 2 & 2 & 2 & 2 & 4 & 10 & 7.15 \\
\hline
\end{tabular}


Seventeen outliers were detected in the data and they were replaced with the fitted values for the model. These outliers represent $7.08 \%$ of the data. This is shown in Table 4-43.

\section{Normality}

Normality was assessed by testing the normality of the Studentized residuals. One of the assumptions of the ANOVA is that the distribution of the residuals is normal. A Tquantile plot $(\mathrm{DF}=113)$, a histogram, and both skewness and kurtosis values were obtained for the data. The residuals of the data are approximately normal for the histogram and quantile plots. The skewness and kurtosis values do not indicate any strong deviation from normality. Figure 4-11, Figure 4-12, and Table 4-44 summarize these results.

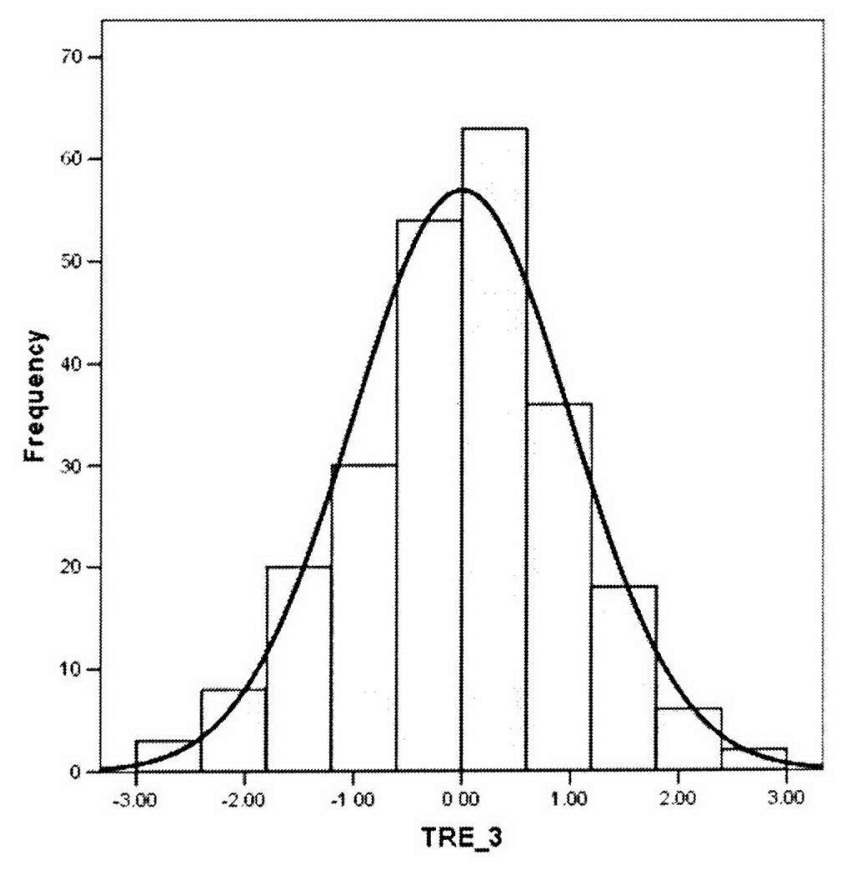

Figure 4-11 Human Subject Test: Experiment 2, Histogram of Studentized Residuals 


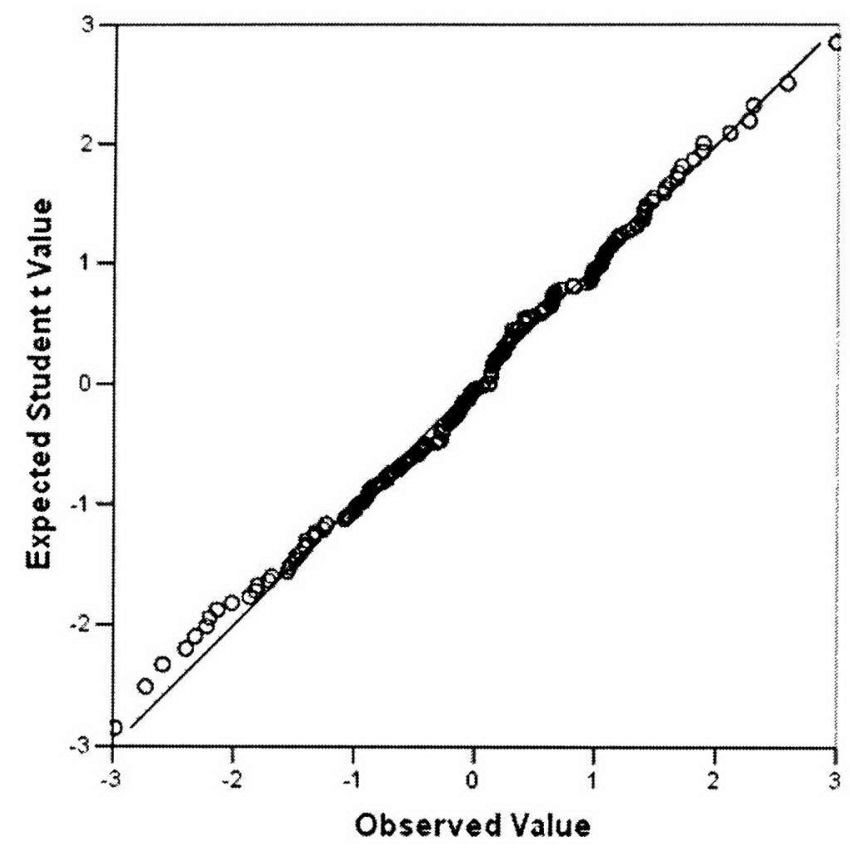

Figure 4-12 Human Subject Test: Experiment 2, T-quantile plot of Residuals

Table 4-44 Human Subject Test: Experiment 2, Skewness and Kurtosis for the Studentized Residuals

\begin{tabular}{|l|r|r|r|r|r|}
\hline & \multicolumn{1}{|c|}{$\mathrm{N}$} & \multicolumn{2}{|c|}{ Skewness } & \multicolumn{2}{c|}{ Kurtosis } \\
\cline { 2 - 6 } & Statistic & Statistic & Std. Error & Statistic & Std. Error \\
\hline TRE_3 & 240 & -.158 & .157 & .317 & .313 \\
Valid N (listwise) & 240 & & & & \\
\hline
\end{tabular}

\section{Homogeneity of Variance}

The homogeneity of variance was tested by applying Levene's statistic to the distribution of each cell of the model. The assumption of equal variances was not met and no transformation could be found that would make the variances equal, but this is still acceptable, as mentioned in the previous section. The results of the Levene test are shown in Table 4-45. 
Table 4-45 Human Subject Test: Experiment 2, Levene's Test for Homogeneity of Variance

\begin{tabular}{|ll|r|r|r|r|}
\hline & \multicolumn{1}{|c|}{$\begin{array}{c}\text { Levene } \\
\text { Statistic }\end{array}$} & \multicolumn{1}{c|}{ df1 } & \multicolumn{1}{c|}{ df2 } & \multicolumn{1}{c|}{ Sig. } \\
\hline CorrectNOT & Based on Mean & 2.169 & 41 & 168 & .000 \\
& Based on Median & .616 & 41 & 168 & .965 \\
& Based on Median and & .616 & 41 & 112.748 & .960 \\
& with adjusted df & 1.884 & 41 & 168 & .003 \\
& Based on trimmed mean & & & \\
\hline
\end{tabular}

\section{ANOVA}

Upon verification of the required assumptions, an ANOVA was applied to the data. The results obtained are shown in Table 4-46. Unfortunately, SPSS ${ }^{\circledR}$ does not allow for contrasts using the mixed linear model analysis. The estimated marginal means for the main effects are shown in Table 4-48, Table 4-49, Table 4-50, and Table 4-51.

Table 4-46 Human Subject Test: Experiment 2, ANOVA Table

\begin{tabular}{|l|r|r|r|r|}
\hline Source & Numerator df & $\begin{array}{r}\text { Denominator } \\
\text { df }\end{array}$ & \multicolumn{1}{c|}{$\mathrm{F}$} & \multicolumn{1}{c|}{ Sig. } \\
\hline Intercept & 1 & 16.395 & 137.018 & .000 \\
Group & 3 & 16.395 & 2.717 & .078 \\
Size & 2 & 97.645 & 6.567 & .002 \\
Eye & 1 & 114.696 & .470 & .494 \\
Method & 1 & 114.696 & 48.450 & .000 \\
Group * Size & 6 & 97.645 & 2.536 & .025 \\
Group * Eye & 3 & 114.696 & 1.929 & .129 \\
Group * Method & 3 & 114.696 & 10.024 & .000 \\
Size * Eye & 2 & 97.645 & 3.835 & .025 \\
Size * Method & 2 & 97.645 & 5.744 & .004 \\
Eye * Method & 1 & 114.696 & .184 & .669 \\
Group * Size * Eye & 6 & 97.645 & 1.594 & .157 \\
Group * Size * Method & 6 & 97.645 & 1.010 & .423 \\
Group * Eye * Method & 3 & 114.696 & .192 & .902 \\
Size * Eye * Method & 2 & 97.645 & 1.047 & .355 \\
Group * Size * Eye * & 6 & 97.645 & 2.054 & .066 \\
Method & & &
\end{tabular}


Table 4-47 Human Subject Test: Experiment 2, Covariates

\begin{tabular}{|c|c|c|c|}
\hline Parameter & & Estimate & Std. Error \\
\hline \multirow[t]{12}{*}{$\begin{array}{l}\text { Repeated } \\
\text { Measures }\end{array}$} & $\begin{array}{l}\text { Var: }[\text { Size }=1.00]^{*}[\text { Eye }=1 . \\
00]^{*}[\text { Method }=1.00]\end{array}$ & $2 E+007$ & 8527971 \\
\hline & $\begin{array}{l}\text { Var: }[\text { Size }=1.00]^{*}[\text { Eye }=1 \\
00]^{*}[\text { Method }=2.00]\end{array}$ & 2733453 & 1267980 \\
\hline & $\begin{array}{l}\text { Var: }[\text { Size }=1.00]^{\star}[\text { Eye }=2 . \\
00]^{*}[\text { Method }=1.00]\end{array}$ & $3 E+007$ & $1 E+007$ \\
\hline & $\begin{array}{l}\text { Var: }[\text { Size }=1.00]^{*}[\text { Eye }=2 . \\
00]^{*}[\text { Method }=2.00]\end{array}$ & 2110188 & 1152646 \\
\hline & $\begin{array}{l}\text { Var: }[\text { Size }=2.00]^{*}[\text { Eye }=1 . \\
00]^{*}[\text { Method }=1.00]\end{array}$ & $2 E+007$ & 6424051 \\
\hline & $\begin{array}{l}\text { Var: }[\text { Size }=2.00]^{*}[\text { Eye }=1 . \\
00]^{*}[\text { Method }=2.00]\end{array}$ & $1 E+007$ & 4894842 \\
\hline & $\begin{array}{l}\text { Var: }[\text { Size }=2.00]^{*}[\text { Eye }=2 \text {. } \\
00]^{*}[\text { Method }=1.00]\end{array}$ & $1 \mathrm{E}+007$ & 5440252 \\
\hline & $\begin{array}{l}\text { Var: }[\text { Size }=2.00]^{*}[\text { Eye }=2 . \\
00]^{*}[\text { Method }=2.00]\end{array}$ & $1 E+007$ & 5353906 \\
\hline & $\begin{array}{l}\text { Var: }[\text { Size }=3.00]^{*}[\text { Eye }=1 . \\
00]^{*}[\text { Method }=1.00]\end{array}$ & 5227318 & 2214492 \\
\hline & $\begin{array}{l}\text { Var: }[\text { Size }=3.00]^{*}[\text { Eye }=1 . \\
00]^{*}[\text { Method }=2.00]\end{array}$ & 7219433 & 2903128 \\
\hline & $\begin{array}{l}\text { Var: }[\text { Size }=3.00]^{*}[\text { Eye }=2 . \\
00]^{*}[\text { Method }=1.00]\end{array}$ & $1 E+007$ & 4720118 \\
\hline & $\begin{array}{l}\text { Var: }[\text { Size }=3.00]^{*}[\text { Eye }=2 . \\
00]^{*}[\text { Method }=2.00]\end{array}$ & 7121300 & 2750932 \\
\hline Subject(Group) & Variance & $3 E+007$ & $1 E+007$ \\
\hline
\end{tabular}

Table 4-48 Human Subject Test: Experiment 2, Estimated Means for Group

\begin{tabular}{|l|c|r|r|r|r|}
\hline & & & & \multicolumn{2}{|c|}{$95 \%$ Confidence Interval } \\
\cline { 5 - 6 } Group & Mean & Std. Error & df & Lower Bound & Upper Bound \\
\hline Control & 19923.333 & 2645.582 & 16.395 & 14325.925 & 25520.742 \\
Myopic & 10307.728 & 2645.582 & 16.395 & 4710.320 & 15905.137 \\
Myopic Astigmat & 13585.968 & 2645.582 & 16.395 & 7988.560 & 19183.377 \\
Keratoconic & 18118.442 & 2645.582 & 16.395 & 12521.033 & 23715.850 \\
\hline
\end{tabular}

Table 4-49 Human Subject Test: Experiment 2, Estimated Means for Size

\begin{tabular}{|l|c|r|r|r|r|}
\hline & & & & \multicolumn{2}{|c|}{$95 \%$ Confidence Interval } \\
\cline { 5 - 7 } Size & Mean & Std. Error & df & Lower Bound & Upper Bound \\
\hline 1.00 & 14827.234 & 1374.691 & 18.999 & 11949.959 & 17704.508 \\
2.00 & 15081.621 & 1371.926 & 18.878 & 12208.893 & 17954.350 \\
3.00 & 16542.749 & 1340.165 & 17.274 & 13718.654 & 19366.844 \\
\hline
\end{tabular}


Table 4-50 Human Subject Test: Experiment 2, Estimated Means for Eye

\begin{tabular}{|l|c|r|r|r|r|}
\hline & & & & \multicolumn{2}{|c|}{$95 \%$ Confidence Interval } \\
\cline { 5 - 6 } Eye & Mean & Std. Error & df & Lower Bound & Upper Bound \\
\hline 1.00 & 15325.852 & 1338.788 & 17.179 & 12503.498 & 18148.205 \\
2.00 & 15641.884 & 1346.634 & 17.593 & 12808.013 & 18475.756 \\
\hline
\end{tabular}

Table 4-51 Human Subject Test: Experiment 2, Estimated Means for Method

\begin{tabular}{|l|c|r|r|r|r|}
\hline & & & & \multicolumn{2}{|c|}{$95 \%$ Confidence Interval } \\
\cline { 5 - 7 } Method & Mean & Std. Error & df & Lower Bound & Upper Bound \\
\hline 1.00 & 13879.718 & 1358.070 & 18.172 & 11028.453 & 16730.984 \\
2.00 & 17088.018 & 1327.186 & 16.609 & 14282.869 & 19893.166 \\
\hline
\end{tabular}

\section{Key Findings}

The main effects of Size and Method were found to be significant, with $p<0.002$ and $\mathrm{p}<0.001$, respectively. The Group*Size, Group*Method, Size*Eye, and Size* Method interactions were found to be significant at the $5 \%$ significance level $(p=0.025, p=0.000, p=0.025$, and $p=0.004$, respectively). This indicates that the method improves the identification of icons for human subjects. This may also indicate that the previous test was not strong enough, in terms of visual acuity, to elicit a response from the subjects that would have been a true measure of their acuity. A more detailed discussion and interpretation of the statistical results presented in this chapter is included in Chapter 5. 


\section{CHAPTER 5}

\section{DISCUSSION}

The results of the experiments that were performed to assess the effectiveness of the proposed algorithm for precompensation of images for display on an LCD panel have shown that images which have been processed resulted in a lower error measure for the software and artificial eye tests. Additionally, for the human subject tests the precompensation method improved the identification of icons in a significant way by increasing the number of icons identified per trial from 9.825 to $11.15(\mathrm{p}<0.005)$.

Experiment one for the software tests was carried out in order to quantify the performance of the proposed algorithm for custom enhancement of digital images based on unique PSFs. The result from the statistical analysis confirmed that the precompensation process was in fact, improving the similarity between the retinal images recorded and the intended undistorted images. There was a significant decrease in the Difference Measure $(\mathrm{p}<0.005)$ when comparing the simulated retinal images without any processing vs. those obtained with the proposed algorithm. The estimated mean for the untransformed Difference Measure was decreased from 1218.4 to 732.4 , indicative of an improvement in similarity. However, the other factor of interest, size, also proved to be significant, with $\mathrm{p}<0.005$. One would expect that the size of the icon would have an impact on the mean similarity because the Difference Measure is an absolute measurement, not a relative measure. In other words, the larger the icon, the more possibility for the raw error measure to be larger, since the measure is not normalized by the mean, for example. The interaction of the method and size factors is not significant, which is encouraging. This indicates that regardless of size, applying the method to the 
images results in a retinal image that is more similar to the intended image. The difference contrast applied to the size factor indicates that every level of the factor size is different from the effects of the previous levels. This finding is reinforced by looking at the S-N-K test on size, shown in Table 4-9.

The encouraging results of the first experiment in the software simulation prompted further verification of the effectiveness of the precompensation. It was decided to perform a repeated measures experiment (treated as a complete randomized block design, blocking on subjects) on twenty unique PSFs, in a way that would be similar to the how the human subjects were to be tested. The results from the statistical analysis yielded interesting findings. For experiment two of the software simulation, all of the factors of interest tested in the ANOVA were found to be significant, including all of the interactions. The method factor resulted in a 54\% decrease in the untransformed similarity factor when comparing blurred retinal images to precompensated retinal images (1598.62 vs. 861.64 , respectively). Additionally, the size*method interaction was found to be significant. For the previous experiment, this interaction was not significant, as mentioned above, whereas for the second software experiment, that is not the case. This may indicate that perhaps the method will work better for certain sizes when testing repeated measures. The interaction plot for size*method (Figure 5-1) reveals that the lack of precompensation does cause the Difference Measure to be slightly higher for certain sizes as compared to the other icon sizes. For size three, it indicates that a lack of precompensation causes the Difference Measure to be higher than the other two icon sizes. 


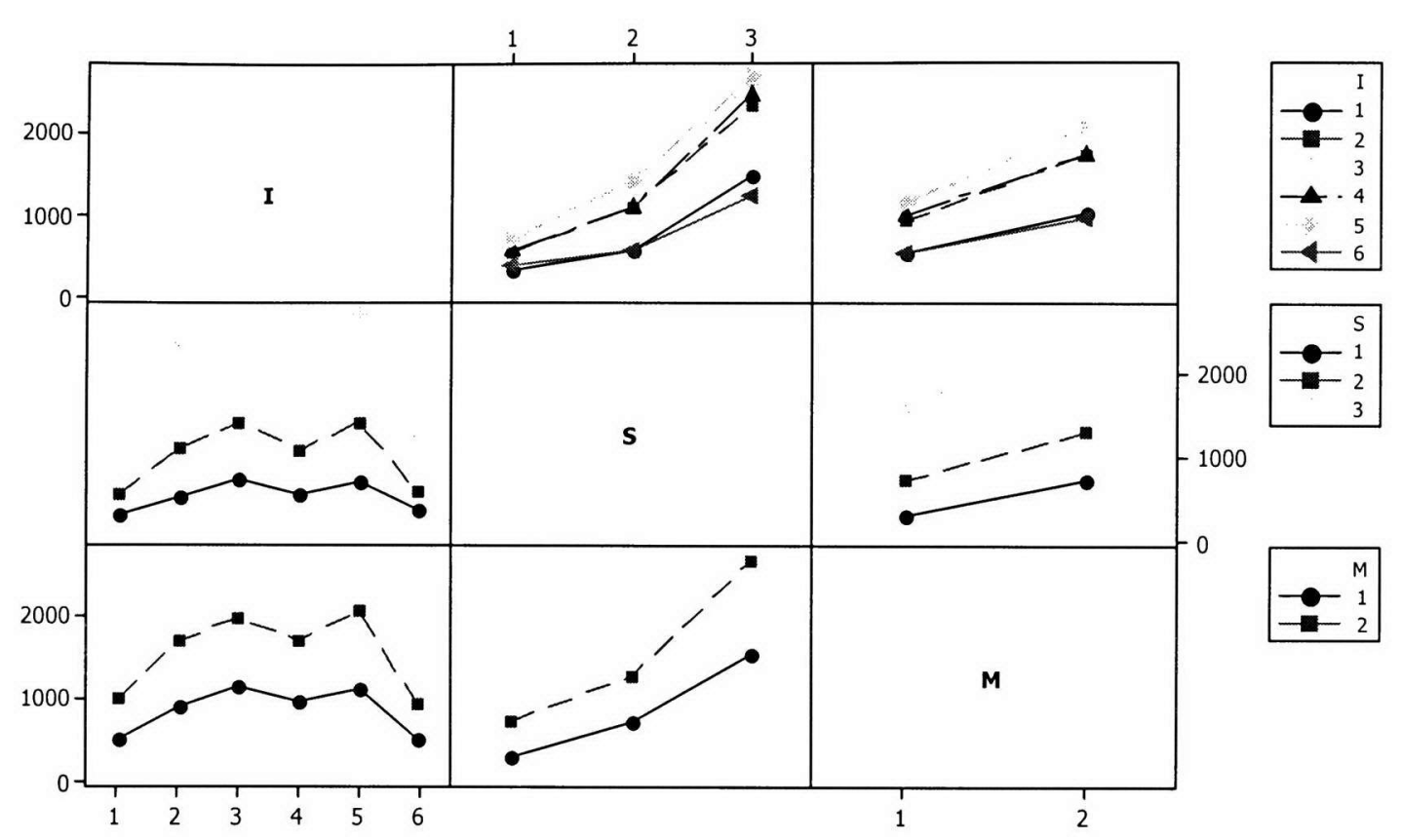

Figure 5-1 Software Test: Experiment 2, Interaction plot matrix (I-icon, S-size, M-method)
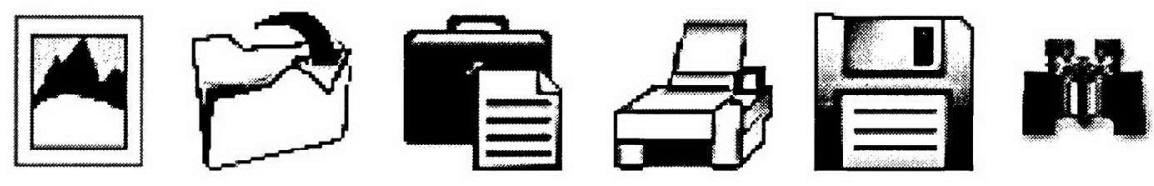

Figure 5-2 Icons used in testing - from left to right, icons one through six

The other interesting interaction is the method*icon interaction. As can be seen from the interaction plot in Figure 5-1, method one resulted in a lower Difference Measure across all icons, although for some icons, the amount of change in similarity due to the method factor is larger for icons $2,3,4,5$, than for icons 1 and 6 . This points the possibility that the precompensation process may perform better for certain icon designs, than for others. Additionally, some icons may have a lower Difference Measure than others, even without precompensation applied, as is evident by the main effects plot for icons, shown in Figure 5-3, and the S-N-K test, shown in Table 4-18. 


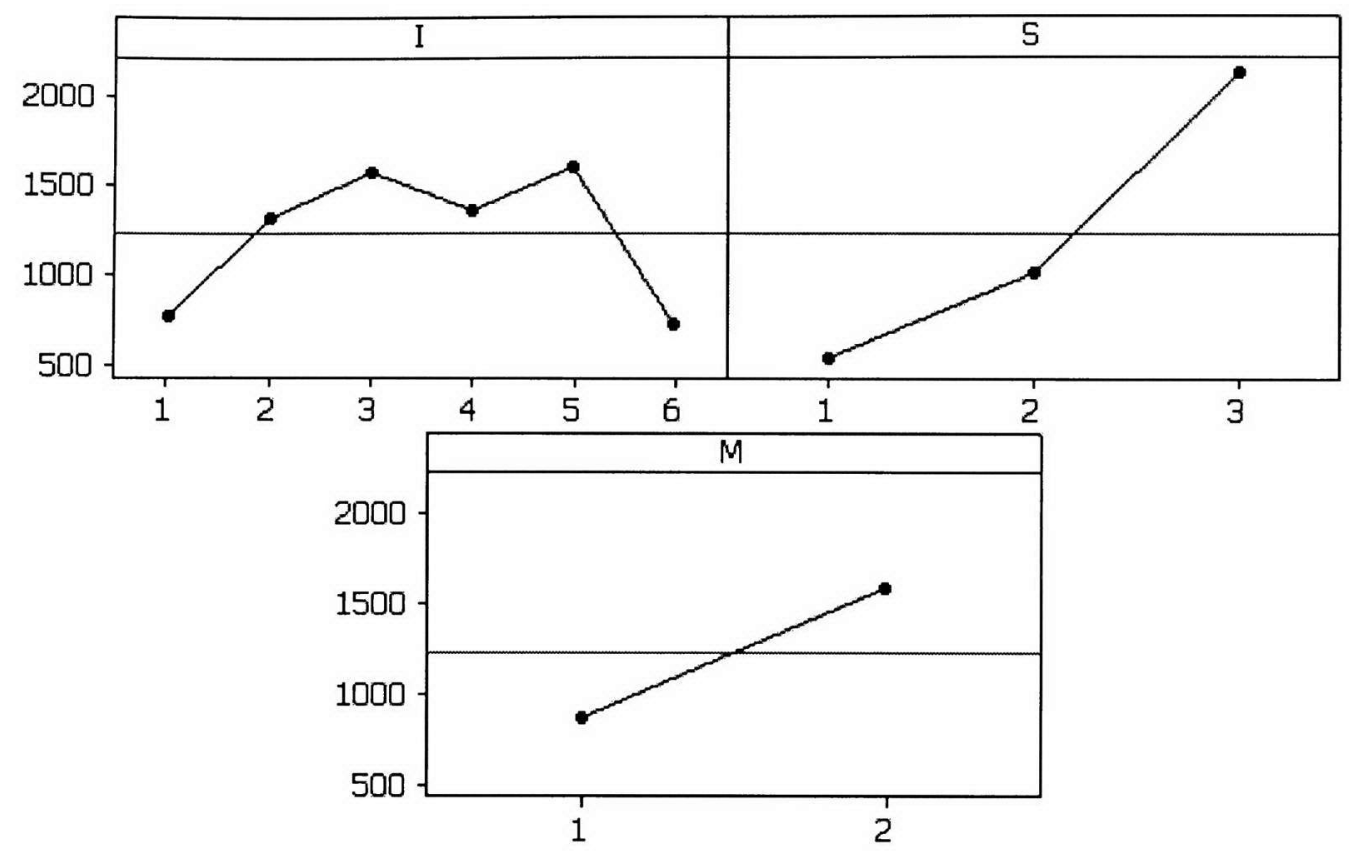

Figure 5-3 Software Test: Experiment 2 - Main effects plots (I-icon, S-size, M-method)

The artificial eye test was the next logical step in testing the proposed algorithm. The artificial eye provided a static, real optical system for allowing the method to be verified. Five lenses, each taken to be a subject, were tested in a way similar the software experiment two. The results agreed strongly with the findings from experiment two of the software tests, in that all the main effects and interactions were significant, even though the five lenses used each had a unique PSF that was not constrained to a simple defocus distortion, as in the software examples. This finding suggests that the precompensation algorithm can successfully improve the similarity of the icons displayed for the camera system having high-order PSFs. The similarity improved, with means of 783.8 vs. 1835.6 for method 1 and 2, respectively. This can be seen in Figure 5-5.

Although there were interactions found, the precompensation algorithm consistently produced a lower Difference Measure across all icons, and sizes. This is 
shown by the icon*method and size*method interactions in Figure 5-4. Interestingly, the icon*size interaction shows a large interaction for cases where icon three is at size four and icon four is at size one. The fact that the icon*size interaction is significant in this way may be indicative of that each unique lens may produce Difference Measures that cause significant variation from the mean, depending on the icon-size combination. This finding prompted an investigation into the outliers that were replaced and, interestingly, 7 of the 9 outliers that were replaced came from lens number 5 .

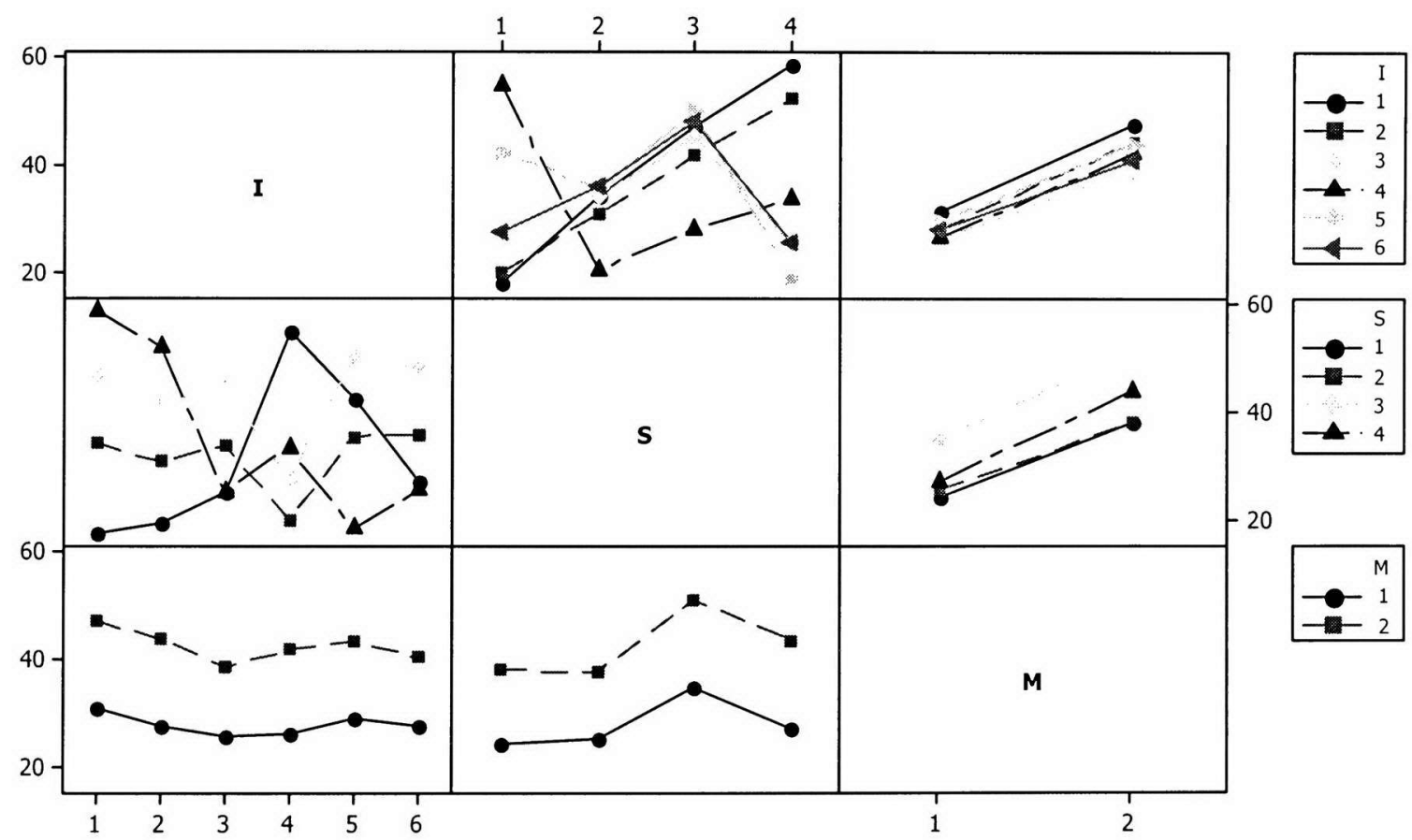

Figure 5-4 Artificial Eye Test: Interaction plot matrix

Upon computing the equivalent ophthalmic prescription from the Zernike polynomial using the method described in [35], it was found that lens 5 had an equivalent prescription of -12.4332 sphere, -1.5244 cylinder at $162.4^{\circ}$. This is far beyond the range of most human eyes, where only about $7 \%$ of myopes have high myopia $(<-6.0$ 
Diopters) [4], leading to the conclusion that the lens itself is not representative of the population of human eyes with low vision. Additionally, it has been found that the larger the PSF (higher amount of sphere), the harder it is to generate an effective precompensation for display on conventional LCD panels. This will be elaborated on further in this section.

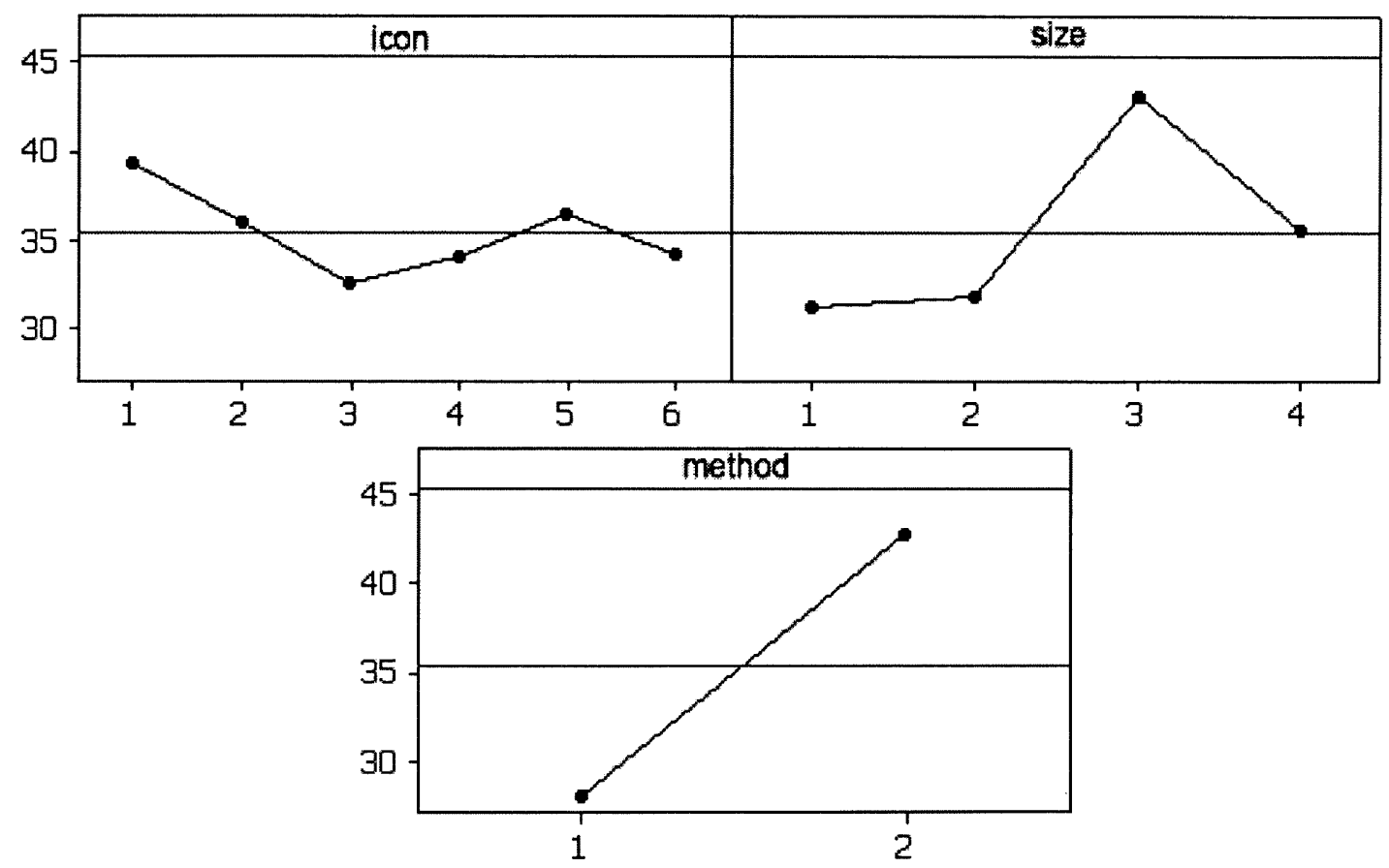

Figure 5-5 Artificial Eye Test - Main effects plots

Helping users with low vision is the ultimate goal of this research work. Thus, two experiments were performed to asses the improvement in vision by means of tasks involving human subjects as participants. The first experiment was designed to assess the improvement in visual acuity, a common measure of visual performance used in ophthalmology [71]. The results for this experiment were surprising, when compared to the previous software and artificial eye test results. The Group factor and the Group*Eye 
and Eye*Method interactions were found to be significant at the 5\% significance level $(\mathrm{p}=0.049, \mathrm{p}=0.043$, and $\mathrm{p}=0.035$, respectively). Although there was significance among groups, it is not a very strong significance, noting that it is only slightly less than the $5 \%$ significance level. This may be evidence that different groups may perform differently in acuity tests, and perhaps even other visual tasks. The interactions are unexpected in that the eyes should not interact with any factor, including group. If these interactions are truly present, this would mean that the responses from the left and right eye would be different, depending on which group and which method is used. Interestingly, the eye factor itself is not significant, which is as expected. Figure 5-6 shows the interaction plot matrix for this experiment.

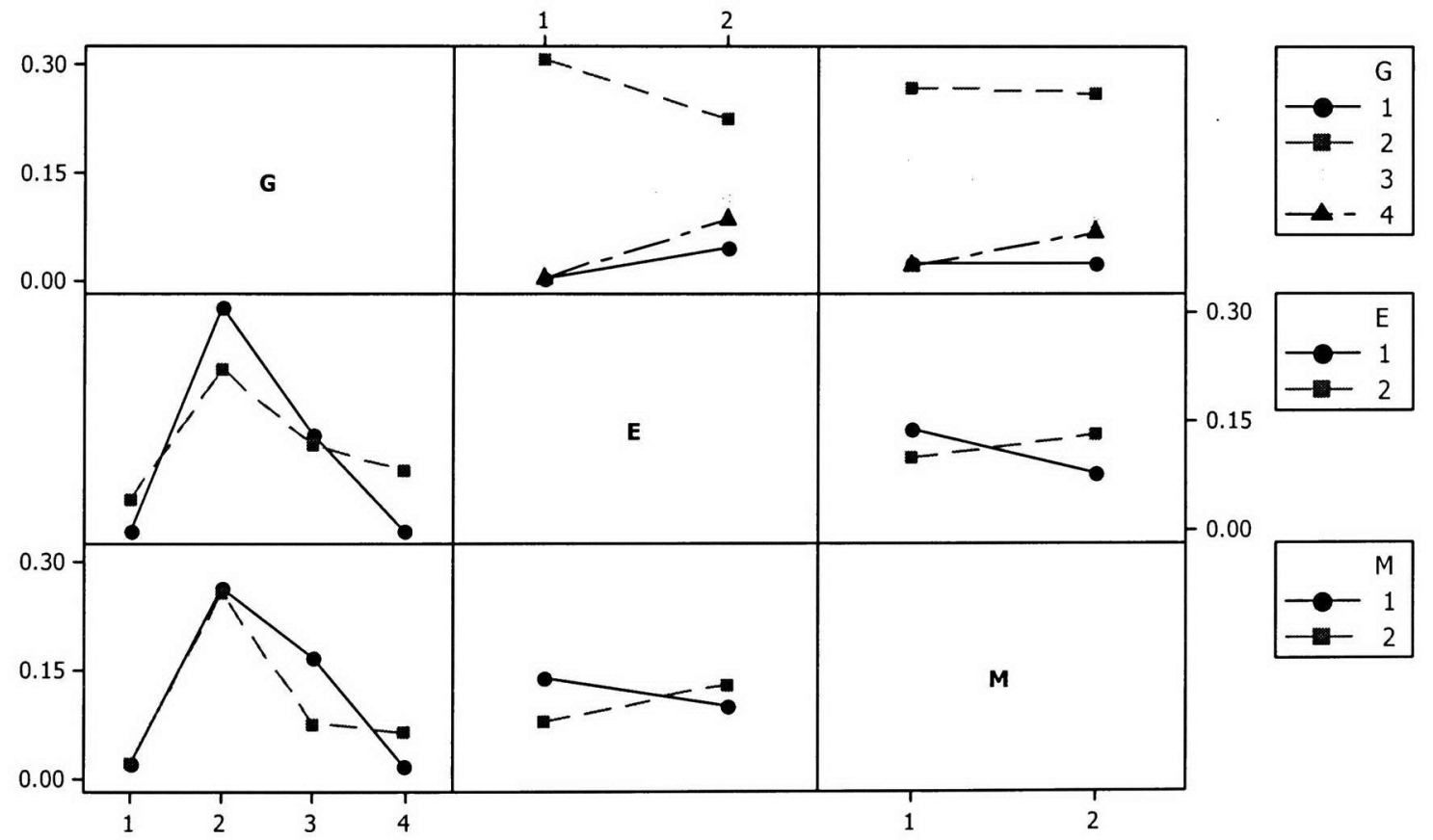

Figure 5-6 Human subject tests: Experiment 1 - Interaction plot matrix 


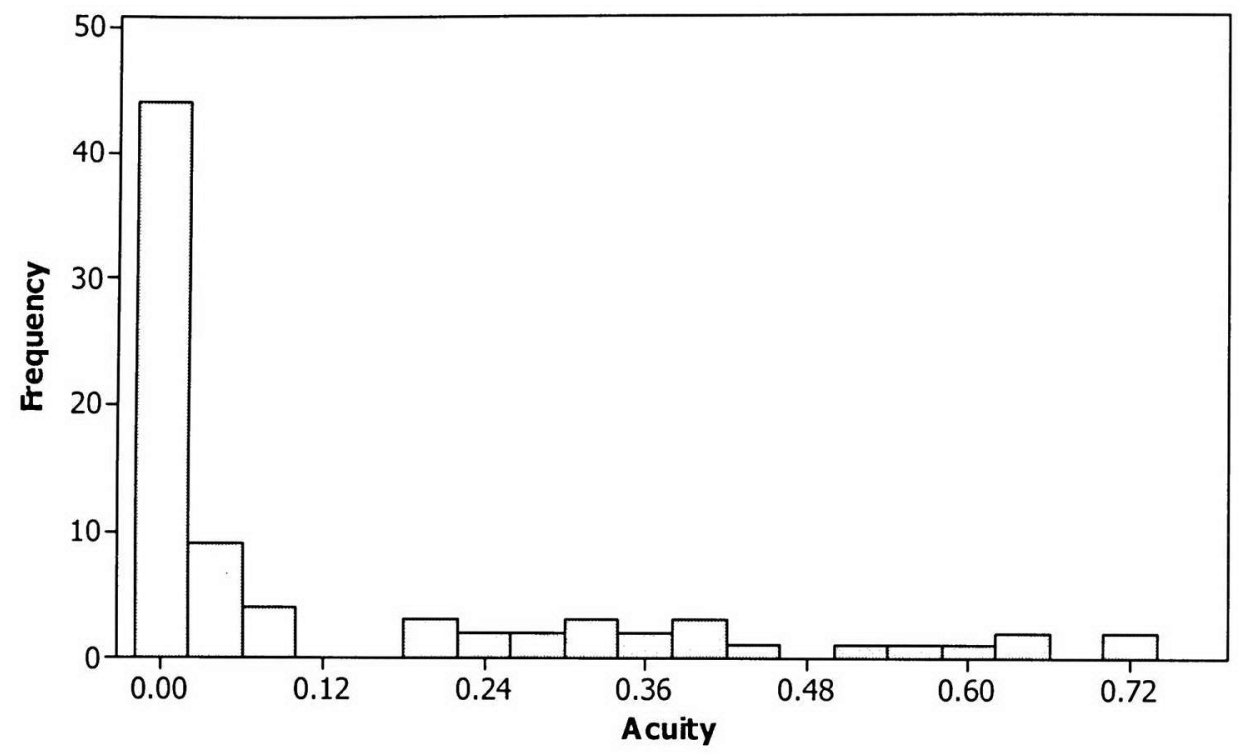

Figure 5-7 Human subject tests: Experiment 1 - Histogram of acuity responses

Additionally, the primary factor of interest, method, is not significant. This is contrary to the findings from the previous experiments. The assumption of the eye test applied to each subject is that at some point, the subject will no longer be able to identify the orientation of the letter E. But if a histogram of the responses is computed (shown in Figure 5-7), it is evident that the visual acuity score for most of the subjects was recorded to be 0.0 . A test on the proportion of visual acuity scores that were equal to 0.0 revealed that at least $50 \%$ of the visual acuity scores were 0.0 , regardless of which method was used. It can be concluded that the acuity test was not challenging enough to clearly reveal specific differences in the visual acuity of the subjects, at the viewing distance used.

The second experiment conducted on the subjects was an icon matching test. The purpose of this test was to evaluate the effectiveness with which the proposed algorithm can improve the identification of icons displayed on-screen. The main effects of Size and Method were found to be significant, with $p<0.002$ and $p<0.001$, respectively. The 
Group*Size, Group*Method, Size*Eye, and Size*Method interactions were found to be significant at the $5 \%$ significance level $(p=0.025, p=0.000, p=0.025$, and $p=0.004$, respectively). This indicates that the method significantly improves the identification of icons. The amount of improvement although significant, is not as large as expected, with the actual mean number of icons identified rising from 9.825 to 11.5 , for icons viewed without and with precompensation, respectively (show in Figure 5-8). Additionally, the number of icons correctly identified goes up as the size of the icons increases, as can be seen in the main effect plot for size, Figure 5-8. This is expected, as the larger the object, the easier it is to identify it.

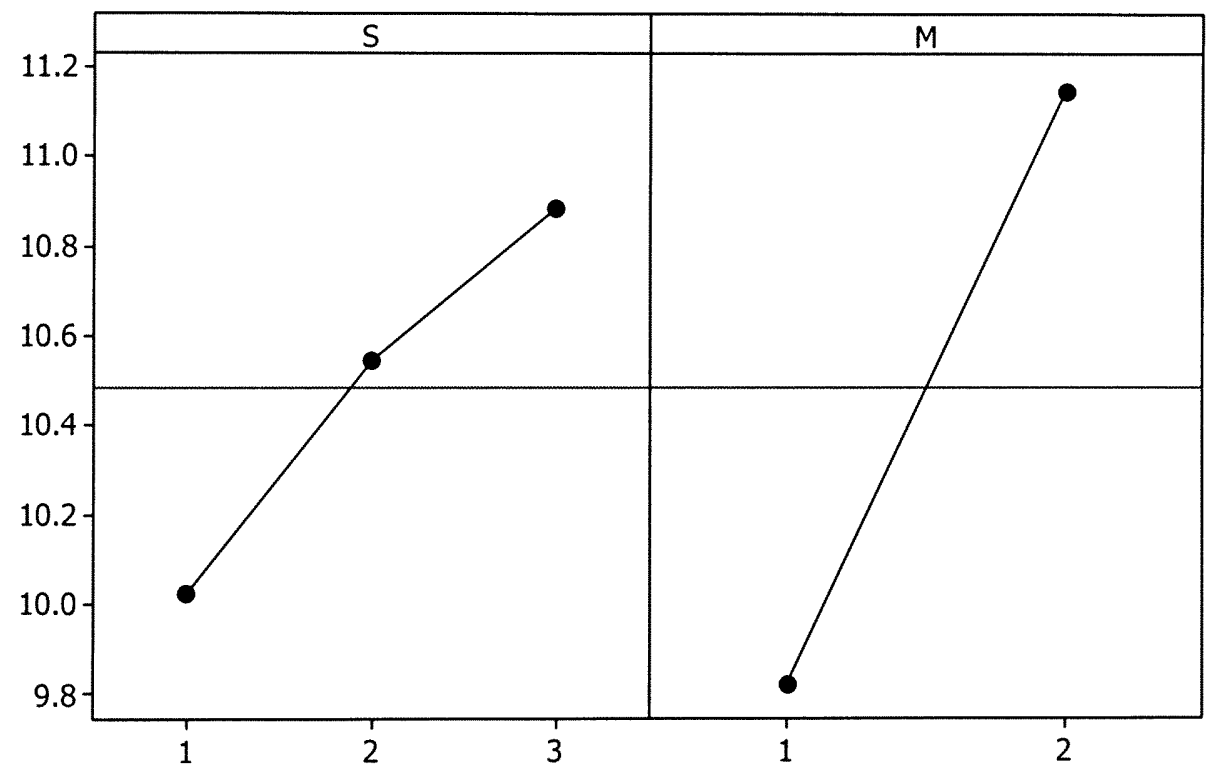

Figure 5-8 Human subject tests: Experiment 2 - Main effects plots (S-size, M-method)

Analysis of the significant interactions reveals some interesting findings. For the group*size interaction, the interaction plot (shown in Figure 5-9) shows that for group one, the control group, there was equal performance across all icon sizes. This is as expected, since the control subjects were chosen to have no significant visual aberrations. 
For group four, the keratocones, there is a slight increase in the number of identified icons as the size increases, although it is not large. This is primarily due to the fact that although the subjects from group four have Keratoconus, their prescriptions (except for the last subject in this group) are similar to the controls category (i.e. their spherical distortion is less severe than -2 Diopters). In other words, for purposes of viewing the computer screen, most of the keratoconic subjects performed nearly as well as the controls, with the exception of subject number 20. For groups two and three, the myopes and myopic-astigmats, the change is apparent. As icons increase in size, the number of icons identified also increases. This suggests that the size of the aberration greatly affects the number of icons that are able to be identified. Keratoconus is a high-order aberration, where as myopia and astigmatism (or a combination) are $2^{\text {nd }}$ order aberrations.

The group*method interaction reveals similar findings. For the control group, the method does not improve the number of icons identified. This is expected because the control group should not need any precompensation to correctly identify the icons. The keratoconic group, as mentioned above, behaves similar to the control group, in that the number of icons identified is not greatly affected by whether or not they are viewing normal or precompensated icons. For groups two and three (myopic and myopicastigmatic), the method was successful in improving the identification of icons by approximately three icons for group two and two icons for group three. This indicates that not all aberrations influence vision equally. This phenomena has been documented in the literature [16]. The last interaction, size*method, reveals that for the largest size icon, the precompensation algorithm does not improve the identification of icons. This is as expected because for large icons, the original acuity requirement implicit in the 
identification task is less stringent and may be met by most subjects in these groups. For the smaller icon sizes, i.e. sizes one and two, the method does improve the identification.

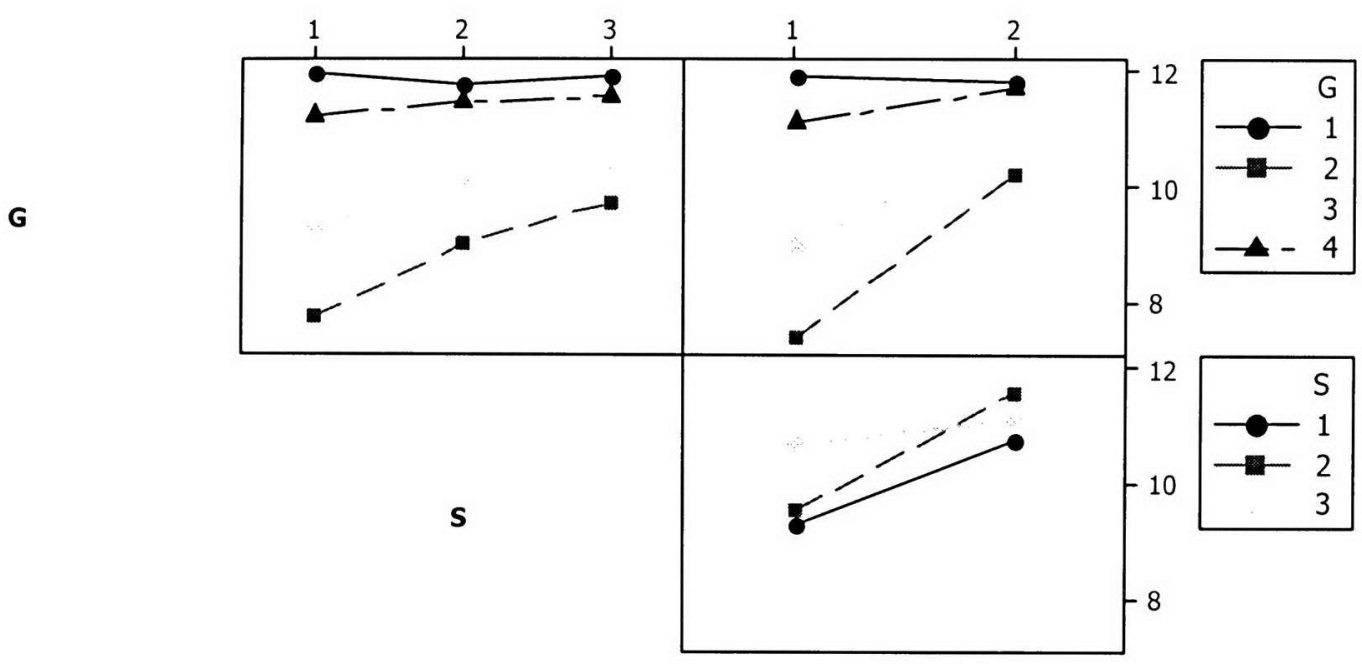

$\mathbf{M}$

Figure 5-9 Human subject tests: Experiment 2 - Group*Size, Group*Method, Size*Method interaction plot matrix

Surprisingly, the method works better in improving the identification of icons by three icons for size two, and two icons for size one. From these findings, it can be inferred that the size of the icon cannot be too small, or the precompensation process will not perform to its potential. This may be due to the fact that smaller icons contain higher spatial frequencies and require a higher display resolution to be correctly displayed. If not, the system will interpolate to the display resolution to allow for display. Conversely, the precompensation process does not help for larger icons, primarily because the spatial resolution of most of the subjects was enough to allow them to identify the large icons, which have more power in the lower spatial frequencies than the smaller icons. 
Although the main factor of interest, method (which indicates whether the proposed algorithm for precompensation is successful in enhancing digital images) did prove to be significant for the icon test, the level of improvement was not as high as expected. Upon further research, three possible causes for this (alone, or in combination) were hypothesized.

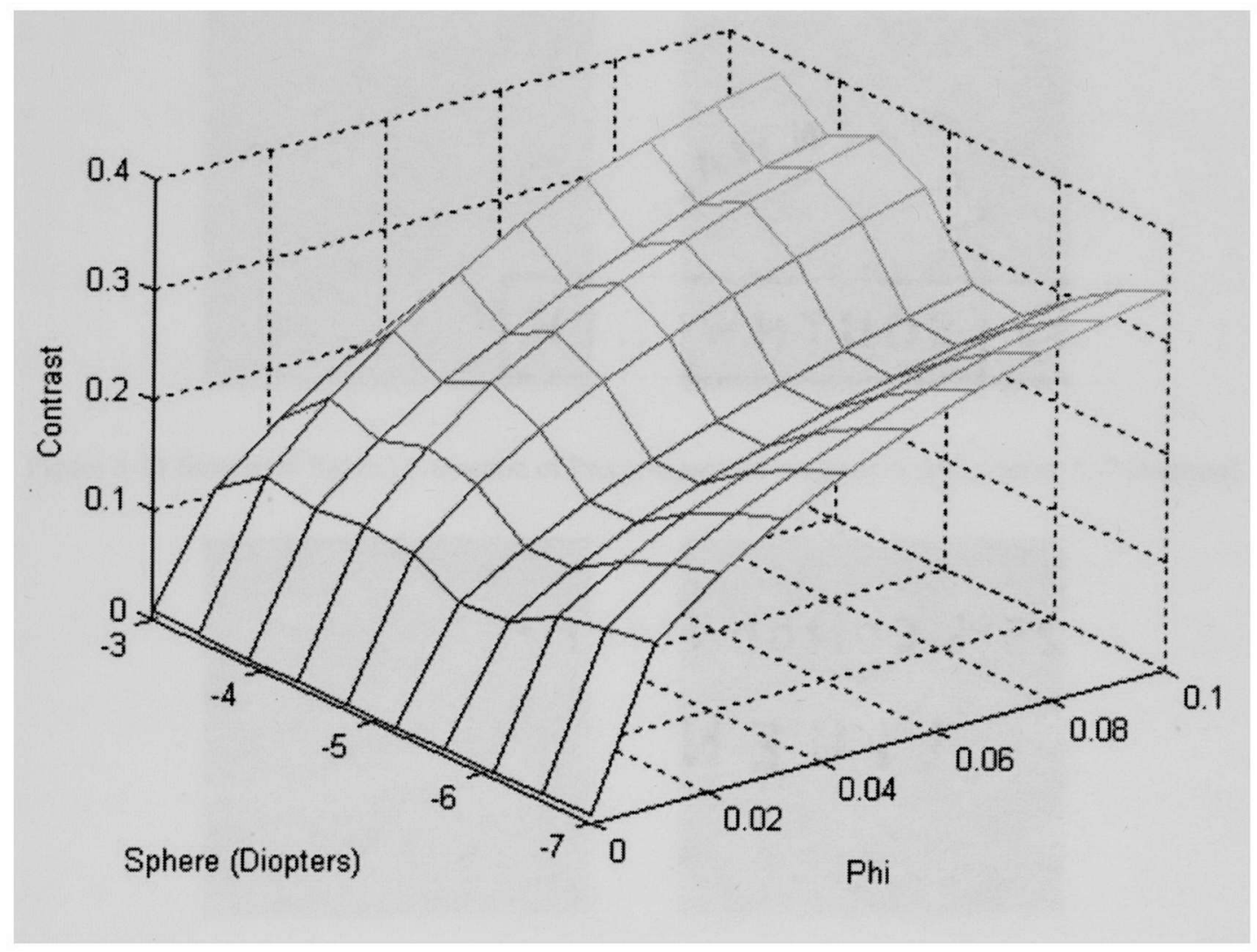

Figure 5-10 Relationship between Defocus, Phi, and Contrast

Firstly, it can be shown that there is a tradeoff between the accuracy of the deconvolution (by means of the $\varphi$ in Equation 3-6) and the amount of contrast present in the image. These factors are directly correlated with the extent of the PSF. Figure 5-10 shows the contrast as a function of $\varphi$ and the amount of defocus (in Diopters), as calculated from Equation 3-6 and 3-27. 
This shows that if a value of $\varphi$ is held constant, as the amount of contrast in the image, (measured by (Lmax - Lmin)/ 255) goes down the amount of defocus goes up. All of the PSFs of the human subjects were in the range of [0 -6.5] Diopters, and since a constant value of $\varphi=0.005$ was used throughout the testing, it is expected that the contrast level would vary depending on their prescription.
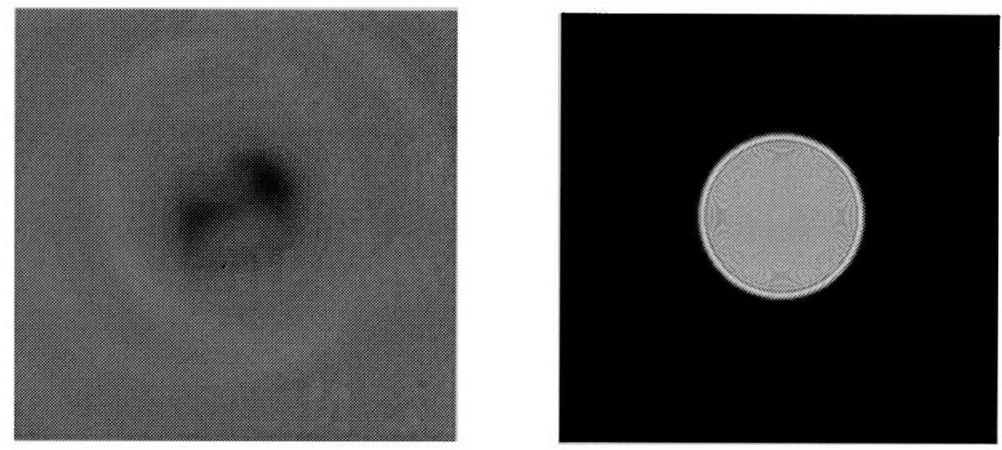

Figure 5-11 Simulated Retinal Projection of Precompensated image $(\varphi=0.005$, sphere $=-7$ Diopters $)$
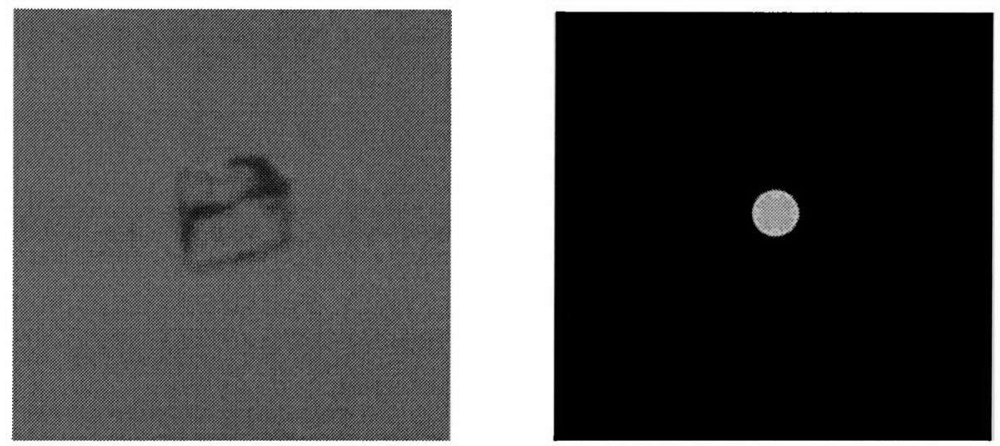

Figure 5-12 Simulated Retinal Projection of Precompensated image $(\varphi=0.005$, sphere $=-3$ Diopters $)$

It can also be shown that for a constant value of $\varphi$, the accuracy of the deconvolution will depend on the spatial extent of the PSF. Figure 5-11 and Figure 5-12 show the same retinal simulation of a precompensated icon for a value of $\varphi=0.005$ using two PSFs (one at $-7 \mathrm{D}$ and the other at $-3 \mathrm{D}$ ). The possibility then arises that the custom 
precompensation was not optimal for each subject because a constant $\varphi$ was used throughout the testing, regardless of prescription.

The second possibility relates to an experimental finding by Steinhauer et al. [11]. They found that even under certain pharmacological agents (tropicamide and dapiprazole), the pupil diameter can vary significantly with a heavy mental load (the icon identification tasks can be considered a heavy mental load as several subjects reported that they forgot the stimulus icons on more that one occasion during the tests). And finally, a last potential disruption of the experimental assumptions stems from the finding by Manny et al. that up to a 0.34 diopter accommodation can be present in the eye, even after the application of tropicamide [25].

The latter two possibilities directly relate to the accuracy of the PSF model used for the precompensation process, which assumed a constant PSF for each subject throughout the duration of the tests. The experimental assumptions did not contemplate possible variability of the pupil diameter or changes in accommodation during the viewing tests with human subjects. The assumption was, upon entering the subject testing phase, that the pharmacological agents applied to the subjects prior to the testing would stabilize the pupil diameter and accommodation, thereby bypassing the need to measure these parameters in real-time.

The ultimate application of this precompensation process would be for standard conditions of human-computer interaction, in which the pupil diameter is expected to vary. These realizations make it clear that practical implementation of the method would require continuous adaptation of the precompensation parameters in real-time. The 
system would require a constant evaluation of the pupil diameter and the associated adjustment of the PSF estimation for precompensation (using a fast method such as the one already available from Campbell [12]) would need to be done for every change in the pupil diameter of the subject. 


\section{CHAPTER 6 \\ CONCLUSIONS AND FUTURE WORK}

A method for providing custom precompensation of visual aberrations for digital images was developed with the following features:

1. Provides parameter-less operation, requires only the knowledge of the PSF of the user. (The PSF for a human eye can be obtained, in practice for the wavefront aberration function with a wavefront analyzer)

2. Provides a significant increase in the ability for users to identify icons that were precompensated on a custom basis

3. Is functional on any PC capable of displaying RGB images on an LCD panel

These features have the potential to provide users with visual aberrations that are otherwise unable to be assisted by conventional means (such as glasses or contact lenses).

This dissertation documents that 1) the image pre-processing method proposed provides compensation for a custom built artificial eye and 2) the pre-processing method provides compensation for human subjects by significantly improving their ability to identify icons displayed on an LCD panel.

Feature one allows the user to easily use the system without having to adjust multiple parameters, requiring only the knowledge of their PSF (which is foreseen to be available from the optometrist office in the near future). Feature two is the advantage of using the method of precompensation for GUIs versus not using any precompensation at 
all. Feature three indicates that the method could, potentially, be implemented on any PC, which would facilitate its wide-spread use.

The interactions from the statistical analysis revealed interesting findings. The software tests revealed that the method will perform differently for different size icons. This highlights the need to optimize the icon size for future use with the precompensation method. Another interesting finding from the software test was that the identification of the icons may depend on the icon design itself. The test revealed that some icon designs benefited from the precompensation more than others, i.e., they displayed a high similarity (retinal projection compared to intended image). Perhaps in the future, icons could be designed taking this into consideration.

For the human subject portion of the experimentation, it was also discovered that the size of the icon displayed impacts the effectiveness of the precompensation process. This is associated to the resolution of the display system in that smaller icons require a higher display resolution to appropriately render all of the detail necessary for identification. It was also found that the amount of sphere and astigmatism present in the optical system, not necessarily the high-order aberrations, may affect the number of icons that a user can identify in viewing tests. The keratoconic subjects, which have high-order aberrations, performed nearly as well as the control subjects, which do not have highorder aberrations, at the viewing distance used in the human subject tests. The other two groups, however, only had second order aberrations. These groups, which had larger sphere and cylinder than the control and keratoconic groups, did not perform as well during the tests. 
Although, the method has proved to increase the number icons identified in humans subjects, that increase is not as much as expected, with an improvement of two to three icons for the myopic and myopic-astigmatic groups. This can be attributed to one or more of the following factors: limitation of the precompensation process to address various PSF sizes with a constant parameter $\varphi$ (Equation 3-6), and the fact that pupil diameter and accommodation have been found to vary under conditions of stress, even under the influence of pharmacological agents. The latter two are directly related to the characterization of the eye in that any change in those parameters will cause the PSF to change. The current method of precompensation does not adjust in real-time, thus limiting its effectiveness if the pupil diameter does, in fact, change between the wavefront measurement and the actual viewing.

These findings open up the possibility for future work by investigating the limitations of the precompensation system in the following ways:

1. Investigate the optimization of the $\varphi$ on a custom basis for various PSFs

2. Investigate methods of providing real-time measurements of pupil diameter and implement real-time adjustment of the precompensation process to make the approach usable under standard viewing conditions (i.e., without the need for the application of pharmacological agents).

3. Investigate optimal icon designs and sizes to maximize the effectiveness of the precompensation process.

Additionally, it would be interesting to incorporate temporal phase information of the light which is used in the LCD display to improve the method. One possibility is to 
use wavefront reconstruction techniques (holography) to produce a precompensated wavefront from a two dimensional LCD display. There is ongoing research of creating digital holograms from LCD displays [9]. Using this technology may help to solve some of the current limitations of the method attributed to low contrast and accuracy of the deconvolution. 


\section{REFERENCES}

[1] M. Alonso Jr., A. Barreto, M. Adjouadi, and J. A. Jacko, "HOWARD: HighOrder Wavefront Aberration Regularized Deconvolution for enhancing graphic displays for visually impaired computer users," Lecture Notes in Computer Science, vol. 4061(pp. 1163-1170, 2006.

[2] M. Alonso Jr., A. Barreto, J. A. Jacko, and M. Adjouadi, "A multi-domain approach for enhancing text display for users with visual aberrations," presented at The Eighth International ACM SIGACCESS Conference on Computers \& Accessibility, Portland, Oregon, pp. 34-39, 2006.

[3] M. Alonso Jr., A. Barreto, J. G. Cremades, J. A. Jacko, and M. Adjouadi, "Image pre-compensation to facilitate computer access for users with refractive errors," Behaviour and Information Technology Journal, vol. 24(3), pp. 161-173, 2005.

[4] Y. B. Dayan, A. Levin, Y. Morad, I. Grotto, R. Ben-David, A. Goldberg, E. Onn, I. Avni, Y. Levi, and O. G. Benyamini, "The changing prevalence of myopia in young adults : A 13-year series of population-based prevalence surveys," Investigative ophthalmology \& visual science, vol. 46(8), pp. 2760-2765, 2005.

[5] C. A. Mertler and R. A. Vannataa, Advanced and Multivariate Statistical Methods: Practical Application and Interpretation, 3rd ed. Glendale: Pyrczak Publishing, 2005.

[6] D. Williams, "2nd generation adaptive optics system for the eye," 2005, http://www.cvs.rochester.edu/williamslab/r secondgenao.html.

[7] H. Cheng, J. K. Barnett, A. S. Vilupuru, J. D. Marsack, S. Kasthurirangan, R. A. Applegate, and A. Roorda, "A population study on the changes in wave aberrations with accomodation," Journal of Vision, vol. 4(4), pp. 272-280, 2004.

[8] B. Chou, "Riding the Wave(front) to Super Vision," in Review of Optometry Online, vol. 140, 2004.

[9] T. Ito and K. Okano, "Color electroholography by three colored reference lights simultaneously incident upon one hologram panel," Optics Express, vol. 12(18), pp. 4320-4325, 2004.

[10] L. Llorente, S. Barbero, D. Cano, C. Dorronsoro, and S. Marcos, "Myopic versus hyperopic eyes: axial length, corneal shape, and optical aberrations," Journal of Vision, vol. 4(4), pp. 288-298, 2004. 
[11] S. R. Steinhauer, G. J. Siegle, R. Condray, and M. Pless, "Sympathetic and parasympathetic innervation of pupillary dilation during sustained processing," International Journal of Psychophysiology, vol. 52(pp. 77-86, 2004.

[12] C. E. Campbell, "Matrix method to find a new set of Zernike coefficients from an original set when the aperture radius is changed " Journal of the Optical Society of America, vol. 20(2), pp. 209-217, 2003.

[13] J. Hjortdal, "Book Review: Wavefront and Emerging Refractive Technology," ACTA Ophthalmologica Scandanavia, vol. 81(6), pp. 681, 2003.

[14] J. Jacko, I. U. Scott, F. Saintfort, L. Barnard, V. K. Emery, T. Kongnakorn, K. Moloney, and B. Zorich, "Older Adults and Visual Impairment: What Do Exposure Times and Accuracy Tell us About Performance Gains Associated With Multimodal Feedback?," CHI Letters, vol. 5(1), pp. 33-40, 2003.

[15] Wavefront Sciences Inc., "Complete Ophthalmic Analysis System User's Manual," 2003.

[16] R. A. Applegate, E. J. Sarver, and V. Khemsara, "Are all aberrations equal?," Journal of Refractive Surgery, vol. 18(5), pp. S556-62, 2002.

[17] R. C. Gonzales and R. E. Wood, Digital Image Processing. New Jersey: Prentice Hall, 2002.

[18] R. Leonard, "Statistics on Vision Impairment: A Resource Manual," vol. 2002, 2002, http://www.lighthouse.org/downloads/researchstats.pdf.

[19] J. N. Parker and P. M. Parker, The official patient's sourcebook on Keratoconus: $A$ revised and updated directory for the internet age. San Diego: Icon Health Publications, 2002.

[20] L. N. Reich and M. Ekabutr, "The Effects of Optical Defocus on the Legibility of the Tumbling-E and Landolt-C," Optometry and Vision Science, vol. 79(6), pp. 389-393, 2002.

[21] T. Daboczi and T. B. Bako, "Inverse Filtering of Optical Images," IEEE Transactions on Instrumentation and Measurement, vol. 50(4), pp. 991-994, 2001.

[22] E. Gerber, "Who's surfing? Internet access and computer use by visually impaired youth and adults," Journal of Visual Impairment \& Blindness, vol. 95(3), pp. 176$181,2001$. 
[23] K. A. Goldberg and K. Geary, "Wave-front measurement errors from restricted concentric subdomains," Journal of the Optical Society of America: A, vol. 18(9), pp. 2146-2152, 2001.

[24] W.-C. Lin, S. A. Toms, E. D. Jansen, and A. Mahadevan-Jansen, "Intraoperative Application of Optical Spectroscopy in the Presense of Blood," IEEE Journal on selected topics in quantum electronics, vol. 7(6), pp. 996-1003, 2001.

[25] R. E. Manny, M. Hussein, M. Scheiman, D. Kurtz, K. Niemann, K. Zinzer, and C. S. Group, "Tropicamide (1\%): An effective cycloplegic agent for myopic children," Investigative ophthalmology \& visual science, vol. 42(8), pp. 17281735, 2001.

[26] W. K. Pratt, Digital Image Processing. New York: Jonh Wiley \& Sons, Inc., 2001.

[27] L. N. Thibos, "Wavefront data reporting and terminology," Journal of Refractive Surgery, vol. 17(5), pp. s578-83, 2001.

[28] A. Field, Discovering Statistics using SPSS for Windows. London: SAGE Publications, 2000.

[29] K. Higuchi, T. Nakano, and S. Yamamoto, "Simulating Human Vision Based on Adaptation and Age-related Visual Changes," $R$ and D Review of Toyota CRDL, vol. 35(2), 2000.

[30] J. A. Jacko, A. B. Barreto, G. J. Marmet, J. Y. Chu, H. S. Bautsch, I. U. Scott, and R. H. Rosa, "Low Vision: The Role of Visual Acuity in the Efficiency of Cursor Movement," presented at Fourth International ACM Conference on Assistive Technologies (ASSETS '00), Arlington, VA, pp. 1-7, 2000.

[31] L. N. Thibos, "Formation and Sampling of the Retinal Image," in Seeing: Handbook of Perception and Cognition, K. K. D. Valois, Ed., 2 ed. San Diego: Academic Press, 2000, pp. 1-54.

[32] L. N. Thibos, R. A. Applegate, J. T. Schwiegerling, and R. Webb, "Standards for reporting the optical aberrations of eyes," in Trends in Optics and Photonics, vol. 35, V. Lakshminarayanan, Ed. Washington, D. C.: Optical Society of America, 2000 .

[33] C. R. Hicks and J. Kenneth V. Turner, Fundamental Concepts in the Design of Experiments. New York: Oxford University Press, 1999.

[34] K. Higuchi, Y. Sakaguchi, K. Sugiyama, and T. Nakano, "Simulating the Human Vision of Elderly for Designing Control Panels," presented at 1999 IEEE 
International Conference on Systems, Man, and Cybernetics, 1999., Tokyo, Japan, pp. 703-708, 1999.

[35] T. O. Salmon, "Corneal Contribution to the Wavefront Aberration of the Eye," in School of Optometry. Bloomington: Indiana University, 1999.

[36] L. N. Thibos, X. Qi, and D. T. Miller, "Vision through a liquid crystal spatial light modulator," presented at 2nd International Workshop on Adaptive Optics for Industry and Medicine, Singapore, pp. 57-62, 1999.

[37] A. Glasser and M. C. W. Campbell, "Presbyopia and the optical changes in the human crystalline lens with age," Vision Research, vol. 38(2), pp. 209-229, 1998.

[38] J. T. Holladay, "Proper method for Calculating Average Visual Acuity," Journal of Refractive Surgery, vol. 13(pp. 388-391, 1997.

[39] J. Liang and D. R. Williams, "Aberrations and retinal image quality of the normal human eye," Journal of the Optical Society of America: A, vol. 14(11), pp. 28732883, 1997.

[40] J. Liang, D. R. Williams, and D. T. Miller, "Supernormal vision and highresolution retinal imaging through adaptive optics," Journal of the Optical Society of America, vol. 14(pp. 2884-2892, 1997.

[41] L. N. Thibos and A. Bradley, "Use of Liquid-Crystal Adaptive-Optics to Alter the Refractive State of the Eye," Optometry and Vision Science, vol. 74(pp. 581-587, 1997.

[42] A. C. Guyton and J. E. Hall, Textbook of Medical Physiology. Philadelphia: Saunders Company, 1996.

[43] R. N. Bracewell, Two-Dimensional Imaging. New Jersey: Prentice Hall, 1995.

[44] R. G. Wilson, Fourier Series and Optical Transform Techniques in Contemporary Optics: An Introduction. New York: John Wiley and Sons, Inc., 1995.

[45] J. Liang, B. Grimm, S. Goelz, and J. F. Bille, "Objective Measurement of wave aberrations of the human eye with the use of a Hartmann-Shack wave-front sensor," Journal of the Optical Society of America, vol. 11(pp. 1949-1957, 1994.

[46] K. Zuiderveld, "Contrast Limited Adaptive Histogram Equalization," in Graphic Gems IV, P. Heckbert, Ed.: Academic Press, 1994, pp. 474-485. 
[47] S. Daly, "The visible differences predictor: an algorithm for the assesment of image fidelity," SPIE: Human Vision, Visual Processing, and Digital Display III, vol. 1666( ), pp. 2-15, 1992.

[48] D. Halliday, R. Resnick, and K. S. Krane, Physics, vol. 2, 4th ed. New York: John Wiley \& Sons, Inc., 1992.

[49] D. Malacara, Optical shop testing, 2nd ed. New York: Wiley, 1992.

[50] E. Peli, "Limitations of Image Enhancement for the Visually Impaired," Optometry and Vision Science, vol. 69(1), pp. 15-24, 1992.

[51] R. H. Webb, C. M. Penny, and K. P. Thompson, "Measurement of ocular wavefront distortion with a spatially resolved refractometer," Applied Optics, vol. 31(pp. 3678-3686, 1992.

[52] B. E. A. Saleh and M. C. Teich, Fundamentals of Photonics. New York: Wiley and Sons, 1991.

[53] R. K. Tyson, Principles of Adaptive Optics. San Diego: Academic Press, 1991.

[54] E. Peli, "New perspectives on image enhancement for the visually impaired," presented at SPIE: Intelligent Robots and Computer Vision IX: Neural, Biological, and 3D Methods, pp. 49-59, 1990.

[55] A. G. Bennet and R. B. Rabbetts, Clinical Visual Optics. London: Butterworths, 1989.

[56] T. B. Lawton, "Improved reading performance using individualized compensation filters for observers with losses in central vision," Ophthalmology (Rochester), vol. 96(pp. 115-126, 1989.

[57] E. Peli, R. B. Goldstein, G. M. Young, and L. E. A. Jr., "Digital image enhancement for the visually-impaired: simulations and results," presented at Proceedings of the 1989 Fifteenth Annual Northeast Bioengineering Conference, Boston, MA., pp. 45-46, 1989.

[58] T. B. Lawton, "Improved word recognition for observers with age-related maculopathies using compensation filters," Clinical Vision Science, vol. 3(pp. 125-135, 1988.

[59] S. M. Raid, "The deconvolution problem: An overview"," Proceedings of IEEE, vol. 74(1), pp. 82-85, 1988. 
[60] J. Serra, Image Analysis and Morphology, Volume 2: Theoretical Advances. London: Academic Press, 1988.

[61] E. Peli, L. E. A. Jr, and G. T. Timberlake, "Computerized image enhancement for visually impaired persons: new technology, new possibilities," Journal of Visual Impairment and Blindness, vol. 80(pp. 849-854, 1986.

[62] E. Peli and T. Peli, "Image enhancement for the visually impaired," Optical Engineering, vol. 23(pp. 47-51, 1984.

[63] G. Walsh, W. N. Charman, and H. C. Howland, "Objective technique for the determination of monochromatic aberrations of the human eye," Journal of the Optical Society of America: A, vol. 1(9), pp. 987-992, 1984.

[64] A. Kenney, "A range of vision: museum accomodation for visually impaired people," Journal of Visual Impairment and Blindness, vol. 77(pp. 325-329, 1983.

[65] E. Peli and J. S. Lim, "Adaptive filtering for image enhancement," Optical Engineering, vol. 21(pp. 108-112, 1982.

[66] J. Serra, Image Analysis and Mathematical Morphology, vol. 1. London: Academic Press, 1982.

[67] G. Covington, "Let your camera do the seeing: the world's fist photography manual for the legally blind.," National Access Center, Washington, DC 1981.

[68] M. Born and E. Wolf, Principles of optics : electromagnetic theory of propagation, interference and diffraction of light, 6th ed. New York: Pergamon Press, 1980.

[69] J. W. Hardy, "Adaptive Optics: a new technology for the control of light," Proceedings of IEEE, vol. 66(pp. 651-697, 1978.

[70] A. N. Tikhonov and V. Y. Arsenin, Solutions of Ill-posed Problems. Washington, D.C.: John Wiley and Sons, 1977.

[71] I. L. Bailey and J. E. Lovie, "New design principles for visual acuity charts," American Journal of Optometry, vol. 57(pp. 378-387, 1976.

[72] B. Platt and R. V. Shack, "Lenticular Hartmann-Screen," Optical Science News Letter, University of Arizona, vol. 5(pp. 15-16, 1971.

[73] J. W. Goodman, Introduction to Fourier Optics. New York: McGraw-Hill Book Company, 1968. 
[74] H. v. Helmholtz, Popular Scientific Lectures. New York: Dover, 1962.

[75] M. S. Smirnov, "Measurement of the wave aberration of the human eye," Biophysics, vol. 7(pp. 766-795, 1962.

[76] H. W. Babcock, "The possibility of compensating astronomical seeing," Publications of the Astronomical Society of the Pacific, vol. 65(pp. 229-236, 1953.

[77] H. v. Helmholtz, Treatise on physiological optics, 3 ed: Optical Society of America, 1924. 


\section{APPENDICES}

\section{APPENDIX A - DERIVATION OF THE POINT SPREAD FUNCTION}

Light is an electromagnetic field that propagates as energy through space in the form of a traveling wave. At any point in space, the amplitude of this field varies as a sine wave in time. The wave propagates outwards from its source, much like the ripples in a pond, and the direction of propagation of the advancing wavefront at any given point in space is specified by a ray emerging from the source that is perpendicular to the wavefront at that point [35].

Outside the paraxial domain of perfect imaging, aberrations exist when the refracted rays from a point source intersect the image plane at different locations, causing the light to be spread out over an area. This distribution of light intensity in the image of a point source, called the point spread function (PSF), is a fundamental characterization of the system. This PSF for two dimensional images is equivalent to the impulse response for a one-dimensional system. The PSF allows for the computation of the image of arbitrary object as the superposition of individual PSFs from every point in the object [31].

The wave theory of image formation states that "the perfect image of a point object is formed by a collapsing hemispherical wavefront with center of curvature located in the image plane" [31]. An aberrated wavefront is characterized by its deviation from this perfect wavefront, or the wavefront error. Thus, the wavefront error can be used to

describe the aberrations introduced by an optical system that produces a wavefront that departs from the ideal wavefront (produced by a "perfect" optical system). 
The Rayleigh-Sommerfeld diffraction integral (Equation A-1) describes the total field at a point R away for a Huygen's wavelet at point S (Figure A-1). The wavefront amplitude $U(S)$ is applied as a weighting factor to the wavelet at point $\mathrm{S}, H(S, R)$.

$$
U(R)=\iint_{\text {Aperture }} U(S) H(S, R) d A
$$

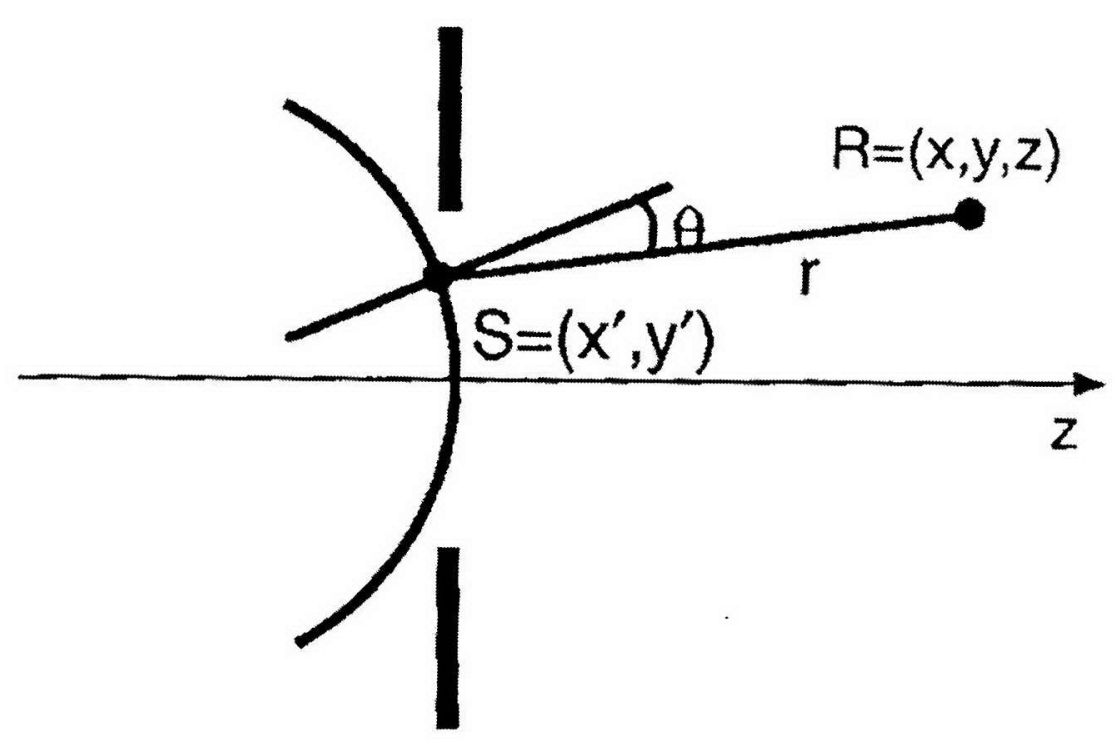

Figure A-1 [31]

The fact that equation A-1 is a superposition resulting from the analysis of diffraction is due to the linearity of the system at hand [73]. Under certain conditions and restrictions, equation A-1 reduces to a convolution integral, immediately allowing the application of the convolution theorem of Fourier analysis [43]. This "transforms the given quantities $\mathrm{U}$ and $\mathrm{H}$ into a corresponding pair of new quantities". This is the domain of Fourier Optics [31] .

Using Fresnel and Fraunhofer approximations (near field and far field approximations, respectively), equation A-1 can be written as 


$$
U(\hat{x}, \hat{y})=C \int_{-\infty}^{\infty} \int_{-\infty}^{\infty} P\left(x^{\prime}, y^{\prime}\right) U\left(x^{\prime}, y^{\prime}\right) \exp \left[-2 \pi \cdot i\left(\hat{x} x^{\prime}+\hat{y y^{\prime}}\right)\right] \cdot d x^{\prime} d y^{\prime}
$$

Excluding the scaling factor $\mathrm{C}$, "the Fraunhofer diffraction pattern is recognized as a two-dimensional inverse Fourier transform of the incident wavefront as truncated by the pupil function" [31] and can be summarized as

$$
U(\hat{x}, \hat{y}) \stackrel{F}{\rightarrow} P(\hat{x}, \hat{y})
$$

where the arrow indicates the direction of the Fourier transform. The amplitude $U$ of the light distribution in a distant image plane due to diffraction of a monochromatic plane wave by an aperture is proportional to the inverse Fourier transform of the aperture's pupil function. Although this analysis seemingly focuses on diffraction, it can be easily applied to real imaging systems by accounting for the focusing properties of the lens through a generalization of the pupil function.

If pupil function is thought of as a two-dimensional filter (varying both amplitude and phase of each point on the emerging wavefront), it captures the effect of the optical system without violating the assumptions that led to equation (A-3). It can be constructed as a complex function, made up of a product of two factors

$$
P\left(x^{\prime}, y^{\prime}\right)=D\left(x^{\prime}, y^{\prime}\right) \exp \left(i k W\left(x^{\prime}, y^{\prime}\right)\right)
$$

where $\mathrm{D}\left(\mathrm{x}^{\prime}, \mathrm{y}^{\prime}\right)$ is attenuation factor, and $\mathrm{W}\left(\mathrm{x}^{\prime}, \mathrm{y}^{\prime}\right)$ is a phase factor termed the wavefront aberration function. The complex amplitude spread function $\mathrm{A}(\mathrm{x}, \mathrm{y})$ in the image plane (including the effects of diffraction) for a point source of light is thus equal to the inverse Fourier transform of the complex pupil function, 


$$
A(x, y) \stackrel{F}{\rightarrow} P\left(x^{\prime}, y^{\prime}\right)
$$

Most detectors of light, such as those found in the retina, respond to the intensity of light, which is real valued and defined as the "time average of the square modulus of the complex amplitude" [28], namely

$$
\operatorname{PSF}(x, y)=|A(x, y)|^{2}
$$

Thus, $\operatorname{PSF}(x, y)$ is called the intensity point spread function, or simply PSF.

When using the preceding arguments for practical applications, it is useful to first normalize the pupil coordinates by the pupil radius when formulating the analytical expression of the pupil function. Once the OTF is derived, the frequency scale may be converted into physical quantities by noting that the cutoff spatial frequency fc set by diffraction is given by

$$
f_{c}=d / \lambda \text { cycles per radian (subtended at the pupil center), }
$$

where $\mathrm{d}$ is the pupil diameter and $\lambda$ is the wavelength [31].

Liang et al. introduced a technique for measuring the wavefront aberrations of the human eye using a Hartmann-Shack sensor [45]. This device, called a "wavefront analyzer", objectively measures the wavefront aberration error in a human eye and is able to generate a polynomial approximation to this wavefront aberration function over a normalized pupil. The output of the wavefront analyzer is a set of Zernike coefficients which when expanded over mesh, approximate the wavefront aberration error. The advantage of this polynomial series is that it is orthogonal over a circle for continuous 
curves, which is extremely beneficial for surface fitting and analysis [35, 53]. A detailed description is given by Born and Wolf [68].

In order to obtain an analytical model of the PSF, or similarly the OTF, the following steps must be followed:

1. Formulate a matrix of values generated from the wavefront aberration function (approximated by the Zernike coefficients), $W\left(x^{\prime}, y^{\prime}\right)$, defined over the pupil area. In other words

$$
W\left(x^{\prime}, y^{\prime}\right)=Z n \cdot z n\left(x^{\prime}, y^{\prime}\right)
$$

where $Z n$ is row a vector of Zernike polynomials, and $z n\left(x^{\prime}, y^{\prime}\right)$ is a column vector where each row is the normalized zernike approximation matched to each term. The units of this function are normalized for ease of calculation. Thus the pupil area can be considered a unit circle for the purposes of calculations. Values of the matrix that lie outside the unit circle are made to be zero.

2. Define $D\left(x^{\prime}, y^{\prime}\right)$, the attenuation factor, as a matrix of values. Again, this function is defined over the pupil area as a unit circle. Typically, this matrix is assigned a value of one within the unit circle, and zero for values that lie outside the unit circle.

3. Using equation A-4, a matrix for $P\left(x^{\prime}, y^{\prime}\right)$, is generated.

4. The autocorrelation is applied to the result from step three, yielding the OTF, $\operatorname{OTF}(u, v)$

5. If the PSF, $\operatorname{PSF}(x, y)$, is desired, the inverse Fourier transform is applied to the result from step four. 


\section{APPENDIX B - ARTIFICIAL EYE AND LENS DESIGNS}

In order to verify the proposed processing, a method of quantifying the results in a physical optical system that functions in a way similar to the optical structure of the human eye is necessary. This "artificial eye" will be used to provide several testing conditions allowing for variation of several parameters including pupil diameter, focal length, and various types of wavefront aberrations introduced via lenses as well as, allow for both objective measurement/characterization of the optical system (i.e. assessment of the wavefront aberration function of the system via the COAS wavefront analyzer) and the ability to 'see' through the optical system via the CCD.

A machine vision camera form PixeLINK ${ }^{\odot}$ was chosen to be the imaging portion of the artificial eye. The PL-A781 machine vision camera (Figure B-1), providing 6.6megapixel resolution from a $7.73 \mathrm{~mm} \times 10.5 \mathrm{~mm}$ CMOS sensor, can capture images of up to $2208 \times 3000$ pixels. For the purposes of this dissertation, the image size was limited to $2208 \times 2208$ to provide square images. The lens mount is a standard C-mount. The device interfaces with the PC through Firewire, providing fast retrieval of captured images. Additionally, the c-mount lens mount simplified the manufacturing of the lens as many c-mount lens parts are available from Edmund Optics.

The remaining specifications, namely a variable aperture, variable focus, and interchangeable high-order lenses, were brought about via assembly of a lens barrel and design of high-order lenses via optical adhesive. The lens barrel was assembled from off the shelf components available through Edmund Optics. An adjustable focusing barrel, 
adjustable iris, and c-mount lens mount were machined and cemented together to create the necessary degrees of freedom needed for testing.

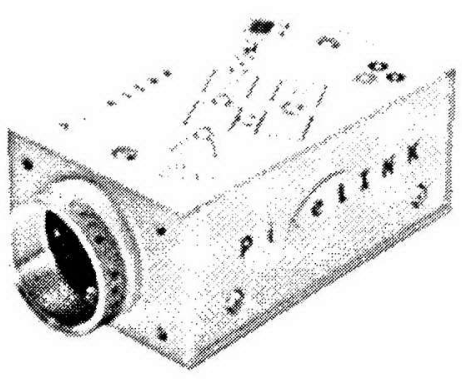

Figure B-1 Pixelink PL-A781 Machine Vision Firewire Camera (http://www.pixelink.com)
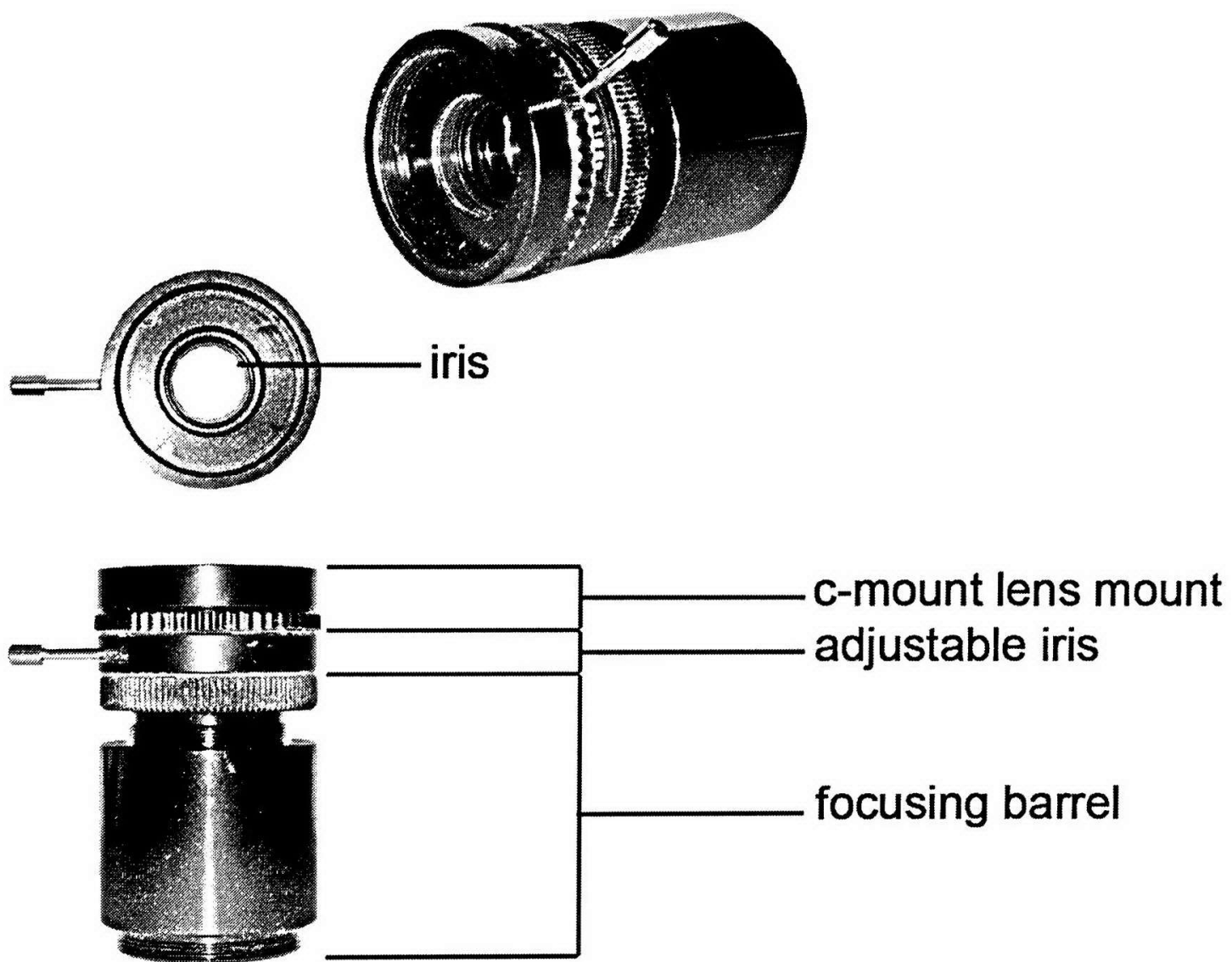

Figure B-2 Lens Barrel 


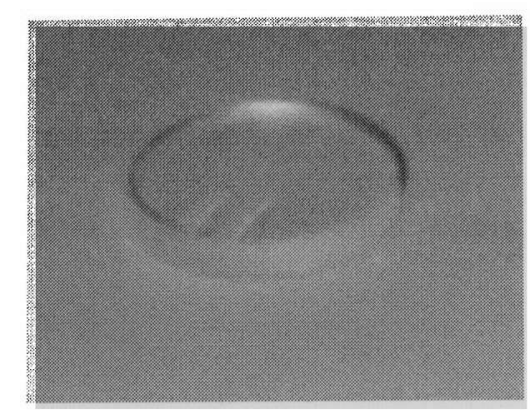

Figure B-3 Example of a high-order lens
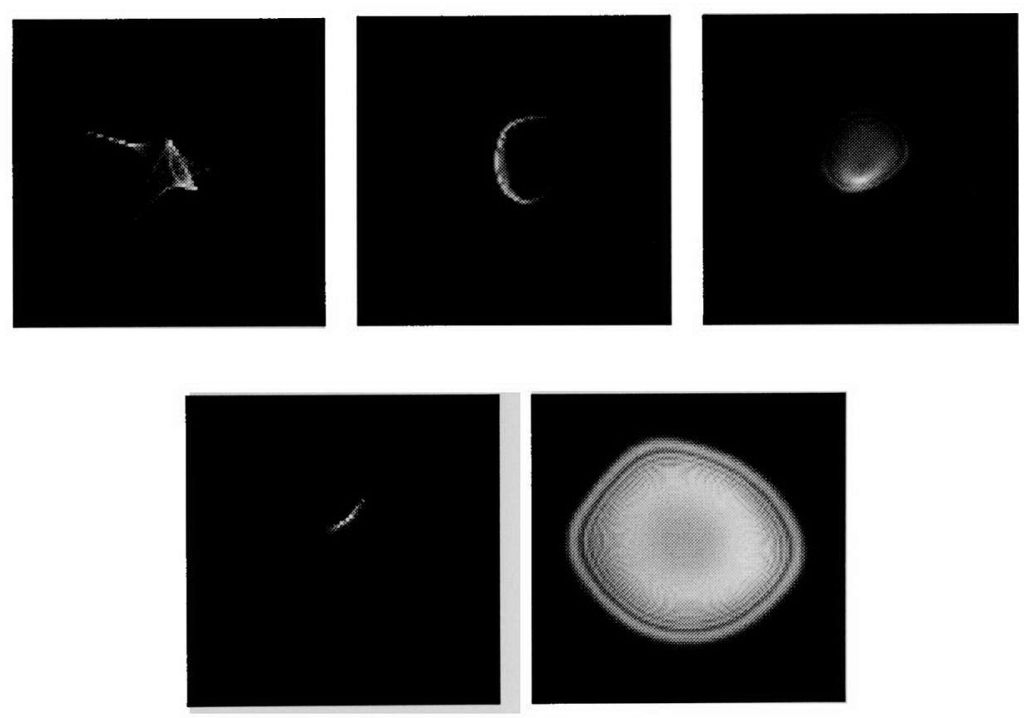

Figure B-4 PSFs of each lens, from left to right and top to bottom, lens 1 through 5

The five lenses were created using a $12 \mathrm{~mm}$ diameter PCX lens from Edmund Optics with a $60 \mathrm{~mm}$ focal length (Edmund Optics part number 32-854). Four of the lenses were used to make unique, high-order aberrations via an optical adhesive coating on the curved surface of the lens. The optical adhesive used was Norland ${ }^{\mathbb{O}}-63$, available from Norland Products ${ }^{\mathcal{O}}$. This is a UV curable adhesive that has similar optical properties as the BK-7 material used in the manufacture of the PCX lenses. A thin coat of Norland63 was applied to each of the four lenses and was allowed to settle for approximately 10 minutes, after which time, the lenses were set to cure under a UV lamp. The irregularities 
caused by manual application of the adhesive to the surface of the lens provided random, high-order aberrations to be used for testing. One of the five lenses remained uncoated to provide only spherical aberrations. The PSFs of the lenses, as measured by a wavefront analyzer, are shown in Figure B-4. 


\section{MEMORANDUM}

To: $\quad$ Dr. Armando Barreto

CC: File

From: Chris Grayson, CIM, Institutional Review Board Coordinator

Date: June 16, 2006

Proposal Tille: Customizable Display for the Visually Impaired.

Approval \# $\quad 063005-00$

The Institutional Review Board of Florida International University has re-approved your study for the use of human subjects. Your IRB approval date is June 12, 2006 and this approval will expire on June 30, 2007. As a requirement of IRB approval you are required to:

1) Provide immediate written notification to the IRB of:

- Any additions to, or changes in the procedures involving human subjects,

- Every serious or unusual or unanticipated adverse event as well as problems with the rights or welfare of the human subjects. Confirmation of receipt of serious Al: reports must be made with the IRB office.

2) Utilize copies of the date stamped consent document(s) for the recruitment of subjects and receive annual renewal of consent documents.

3) Receive annual review and re-approval prior to your expiration date.

\section{Special Conditions: N/A}

Please note your approval number is indicated above. For further information, you may contact the IRB Coordinator by email at irbiacucafiu.edu or visit the OSRA - Iluman Subjects website at wworostaliu.edu. 


\section{INFORMED CONSENT}

\section{Project Title: On-screen Deconvolution to Facilitate Computer Access for Users with Visual Impairments Involving Iligher-Order Wavefront Aberrations}

\section{Funding Source: National Science Foundation}

Principal Investigator:

Pancla R. Oliver, O.D., M.S.

Associate Protessor of Optometry

College of Optometry

Nova Southeastern University

$3200 \$$ University Dr.

Ft. Lauderdale. FI, 33328

954.262 .1476

\section{Co-Investigator:}

Armando Barreto, Ph.D.

Associate Professor, ECE \& BML

Forida International University

10555 West Flagler Street,

Room EAS-3956

Miami, FL 33174

305-348-3711

Institutional Review Board, Office of Grants and Contracts, Nova Southeastern Iniversity: 954-262-5369

\section{Deseription of the Study: \\ Purpose:}

You are being akked wo volunter in a research study. The purpose of this study is to examine an alternate solution wo the problem of enlancing your ability to see while looking at a computer display. You are being asked to participate voluntarily in this project that will include about 40 persons.

\section{Procedure:}

The project is divided into two sessions, each lasting 1 to 2 hours. The first session will consist of many of the lests in a complete routine eye examination. It will take about one to wo hours to complete the examination in the first visit. Your eye examination will include dilating your eyes to perlorm an evaluation of the optical properties of your eye. In the second session you will be asked to use a computer to perform traditional computer-based tasks. You may also be taught to use a computer designed for people who have impaired vision. You will use the computer to perfom tasks sueh as open files, copy files, elick on ieons, move icons, cte. Somene will be observing you while you use the computer. It will take about one to two hours for you to complete the computer session in your second visit. The entire time for all these procedures should be approximately 2 to 4 hours during two visits to the Nova Southeastem Univeristy Eye Institute ar Davie. 


\section{Risks/Benefits to the Participant:}

There are minimal risks and discomforts that are the same for anyone using a computer and having a routine eye examination. Dilation is part of a routine eye examination. When the eyes are dilated, you may have difficulty reading, sensitivity to light and be unable to drive for a period of several hours after the drops are instilled and your eyes will return fo nomal at the end of this period. In the very unlikely event that you are injured during participation in thes study, you will be provided immediate medical attention, at your own expense. In the very unlikely case of injury, the Principal Investigator, Pamela R. Oliver, OD, MS, should be contacted immodiately at (954) 262-1476 office or (954) 730-3911 pager.

Your participation in this study may have no direct benefits to you but will help us to understand how computers nay be better designed to help people with decreased vision. You may learn about certan types of feedback that enable you to work more efficiently and eflectively.

\section{Costs and Payments to the Participant:}

You will be paid $\$ 25.00$ for your first session and $\$ 75.00$ for your second session during your participation in this study. If you withdraw early, your compcnsation will be prorated based on the amount of time you participated. Other than any payments specifically stated in the consent form, there is no other compensation avalable for your participation in this research.

You will not be charged for any testing done solely for the purpose of this study.

\section{Confidentiality and Privacy:}

The lollowing procedures will be followed to keep your personal information confidential in this study: Your consent to participate in this study includes consent for the investigator and her assistants to review all your medical records as may be necessary for the purposes of the study. All information obained in this study is stricty confidential unless disclosture is required by law. To protect your privacy, your records will be kepi under a code number rather than by name. Your records will be kept in locked files and only study staff will be allowed to lowk at them. Your name and any other fact that might point to you will not appear when results of this study are presented or published. If any video or audio recording occurs, the tapes will be storcd in a locked cabinet. and will only be accessible to the study investigators. Onee the study has been completed, the tapes will be retained for archival purposes.

\section{Use of Protected IIealth Information (PIII):}

As part of this study, you are being requested to authorize Dr. Pamela R. Oliver, Dr. Perla Najman or Dr. Annette Bade, usually the researehers access to your eye health records at the NSU Fye Institute at Davie, Broward andor North Miami Beach. The purpose of this authorization is to allow the researcher to get the following specific information to be used as part of this research study. This information includes: diagnoses, management including glasses and contact lens prescriptions, comeal topography, refraction, visual acuity, and contrast sensitivity results. You may revoke this authorization at any time by providing a signed written statement that you are revoking the authorization to any of the investigators. Four treatment at NSU will not be effected in any way by your refusal to give this authorization. You will nol be able to participate in the study procedures if you decide that you will not give authorization. If 
You allow this transfer of information from your medical file. lederal or state law will no lenger protect this information and. thus, it is possible that this information could be redisclosed Howeser. we will protect the confidentality of this information as discussed in the Confidentiality sextion. You have the right to refuse to sign this authoriation and informed consent. This will not affect your treatment in any manner.

Your Plll will be shared with any person or agency when required by law, and by:

- The research team for this study

- The spensor(s) of this study, the National Science Foundation

- Nova Southeastem University Institutional Review Board and or the federal Oflice of Human Research Protections for the purpose of assessing complance associated with the conduct of this study.

Use and disclosure of your health information will be necessary for an indetinite period of time:

I understand that I have the right to inspect or copy my Protected Health Intormation to be used or disclosed as permitted under federal and state law (whichever grants the greater acecss rights) or to refuse to sign this agreement. There is no restriction to these rights because of participation in this study.

When you sign the consent form at the end, it means that you have read this section and authorize the use and or disclosure of your personal healh information in the manner explained above:

\section{In Case of Injury/llarm}

The Principle Investigator or her research assistants will be present to monitor your safety. however if you are injured as a result of being in this study. please contact Dr. Pamcla $R$. Oliver at 954-262-1476. Neither the Principal Investigator nor Nova Southeastem University has made provison for payment of costs associated with injury resulting from participation in this stud:

\section{Participant's Rights to Withdraw from the Study:}

- Your participation in this study is voluntary. You do not have to be in this study if you don't want to be.

- You have the right to change your mind and leave the study at any time willout giving any reason, and without penalty.

- Any new information that may make you change your mind about being in this study will be given to you.

- You will be given a copy of this consent form to keep.

- You do not wave any of your legal rights by signing this consent form.

- If you do withdraw, it will not effect your treatment at the Eye Institute in any way. If you choose to withdraw, you may request that any data, which has been collected. will be destroyed unless prohibited by state or federal law. 
Project Title: On-screen Deconvolution to Facilitate Computer Access for Users with Visual Impairments Involving Iligher-Order Wavefront Aberrations

\section{Voluntary Consent by Participant:}

1 have read this consent form, or it has been read to me, and 1 fully understand the contents of this document and voluntarily consent to participate. All of my questions concerning this research have been answered. I hereby agree to participate in this rescarch study. If I have any questions in the future about this study the investigator, Dr. Pamela R. Oliver will answer them. I also voluntarily agree to the release of my PHI as described in this document. A copy of this form has been given to me. This consent ends at the conclusion of this study.

Participant's Name

Participants Signature

Date

Authorized Representative

Date

Authority of Representative is based on:

Witness" Sigmature

Datc 


\section{MIGUEL ALONSO JR}

February 15, 1979

$1997-2001$

2001-2003

2003-2006

2006-2007

2000

2001-2002

$2001-2007$
Born, Miami, FL

B.S., Computer Engineering Florida International University Miami, FL

M.S., Computer Engineering

Florida International University

Miami, FL

NSF Graduate Research Fellow

National Science Foundation

Arlington, VA

FIU Dissertation Year Fellow

Florida International University

Miami, FL

Engineering Intern

G.E. Medical Systems

Milwaukee, WI

Engineer

CPS Products, Inc.

Miami, FL

Research Assistant

DSP Lab, FIU

Miami, FL

\section{PUBLICATIONS AND PRESENTATIONS}

M. Alonso Jr. and A. Barreto, "Polychromatic considerations for the compensation of the wavefront aberration of the human eye," Biomedical Sciences Instrumentation, vol. 43, pp. 218-223, 2007.

M. Alonso Jr., "A method for enhancing digital information displayed to computer users with visual refractive errors via spatial and spectral based processing," ACM SIGACCESS Newsletter, Issue 84, pp. 22-25, 2006. 
M. Alonso Jr., A. Barreto, M. Adjouadi, and J. A. Jacko, "HOWARD: High-Order Wavefront Aberration Regularized Deconvolution for enhancing graphic displays for visually impaired computer users," Lecture Notes in Computer Science, vol. 4061, pp. 1163-1170, 2006.

C. Chin, A. Barreto, and M. Alonso Jr., "Electromyogram-based Cursor Control System for Users with Motor Disabilities," Lecture Notes in Computer Science, vol. LNCS 4061, pp. 905-912, 2006.

M. Alonso Jr., A. Barreto, J. G. Cremades, J. A. Jacko, and M. Adjouadi, "Image precompensation to facilitate computer access for users with refractive errors," Behaviour and Information Technology Journal, vol. 24(3), pp. 161-173, 2005.

M. Alonso Jr., A. B. Barreto, M. Choudhury, and J. A. Jacko, "Software-based Compensation of Visual Refractive Errors of Computer Users," Biomedical Sciences Instrumentation, vol. 41, pp. 229-234, 2005.

M. Choudhury, A. B. Barreto, and M. Alonso Jr., "A Bioinstrumentation System for the Identification of EEG Correlates of Tinnitus," Biomedical Sciences Instrumentation, vol. 41, pp. 169-174, 2005.

M. Alonso Jr. and A. Barreto, "An Affordable Platform for Learning Real-Time Adaptive Signal Processing," International Journal of Engineering Education, vol. 20(1), pp. 39-45, 2004.

M. Alonso Jr. and A. Barreto, "An Improved Method of Pre-Deblurring Digital Images Towards the Pre-Compensation of Refractive Errors," WSEAS Transactions on Computers, vol. 3(2), pp. 487-492, 2004.

M. Alonso Jr. and A. B. Barreto, "An Image Processing Approach to Pre-compensation for Higher-Order Aberrations in the Eye," Journal of Systemics, Cybernetics and Informatics, vol. 2(3), pp. 14-17, 2004, ISSN: 1690-4524.

M. Alonso Jr., A. B. Barreto, and J. G. Cremades, "Image Pre-Compensation to Facilitate Computer Access for Users with Refractive Errors," ACM SIGACCESS Accessibility and Computing, vol. 78(78), pp. 126-132, 2004.

M. Alonso Jr. and A. Barreto, "Digital Image Processing for Pre-Compensation of HighOrder Aberrtions of the Human Eye," Biomedical Sciences Instrumentation, vol. 39, pp. 99-104, 2003.

M. Alonso Jr. and A. Barreto, "Real-Time Computer Station for the Teaching of Adaptive Signal Processing," Computers in Education Journal, vol. 13(1), pp. 2-9, 2003. 